Equações elípticas com não linearidades críticas e perturbações de ordem inferior

Maycon Sullivan Santos Araújo 



\title{
Equações elípticas com não linearidades críticas e perturbações de ordem inferior
}

\author{
Maycon Sullivan Santos Araújo
}

Orientador: Prof. Dr. Eugenio Tommaso Massa

Dissertação apresentada ao Instituto de Ciências Matemáticas e de Computação - ICMC-USP, como parte dos requisitos para obtenção do título de Mestre em Ciências - Matemática. EXEMPLAR DE DEFESA 
Ficha catalográfica elaborada pela Biblioteca Prof. Achille Bassi e Seção Técnica de Informática, ICMC/USP, com os dados fornecidos pelo(a) autor(a)

\begin{tabular}{|c|c|}
\hline \multirow[t]{3}{*}{ A658e } & $\begin{array}{l}\text { Araújo, Maycon Sullivan Santos } \\
\text { Equações elipticas com não linearidades críticas e } \\
\text { perturbações de ordem inferior / Maycon Sullivan } \\
\text { Santos Araújo; orientador Eugenio Tommaso Massa. - } \\
\text { - São Carlos, } 2015 \text {. } \\
\quad 92 \text { p. }\end{array}$ \\
\hline & $\begin{array}{l}\text { Dissertação (Mestrado - Programa de Pós-Graduação } \\
\text { em Matemática) -- Instituto de Ciências Matemáticas } \\
\text { e de Computação, Universidade de São Paulo, } 2015 \text {. }\end{array}$ \\
\hline & $\begin{array}{l}\text { 1. Equações diferenciais parciais elípticas. } 2 . \\
\text { Equações diferenciais parciais. } 3 . \text { Problemas com } \\
\text { expoente crítico. 4. Métodos variacionais. I. Massa, } \\
\text { Eugenio Tommaso, orient. II. Título. }\end{array}$ \\
\hline
\end{tabular}


Meu amor, disciplina é liberdade

Compaixão é fortaleza

Ter bondade é ter coragem

Lá em casa tem um poço, mas a água é muito limpa.

- "Há tempos" Legião Urbana. 


\section{Agradecimentos}

Agradeço acima de tudo a meus pais, José Carlos Oliveira Araújo e Terezinha Maria dos Santos Ararújo, por todo esforço e dedicação em fazer de mim a pessoa que sou hoje.

Agradeço a meu orientador, prof. dr. Eugenio Tommasso Massa, por tudo que ele se dispôs a me ensinar referente à pesquisa matemática, mas, principalmente, pela paciência em fazê-lo.

Agradeço a meu orientador de graduação, prof. dr. Paulo de Souza Rabelo, por ter me iniciado nas EDP's Elípticas, por ter, em alguns momentos, me encorajado a superar minhas limitações e, principalmente, por acreditar em mim ao ponto de me incentivar a trilhar caminhos longíquos na busca de incrementar minha formação acadêmica.

Agradeço à CAPES pelo auxílio financeiro.

Agradeço a todos os professores e colegas de estudos que, durante toda minha carreira acadêmica até aqui, me ajudaram de alguma modo em minha formação acadêmica.

Agradeço também a todas aquelas pessoas que, mesmo não estando diretamente relacionadas à carreira matemática, deram sua contribuição me apoiando me desejando sorte e força dentre outras coisas. 


\section{Resumo}

Neste trabalho, tivemos como objetivo estudar a existência de soluções fracas não triviais para o problema elíptico com não linearidade crítica

$$
\begin{cases}-\Delta u=\lambda u+u_{+}^{2^{*}-1}+g\left(x, u_{+}\right)+f(x), & \text { em } \Omega \\ u=0, & \text { sobre } \partial \Omega\end{cases}
$$

onde $\Omega$ é um domínio limitado com fronteira suave em $\mathbb{R}^{N}$, com $N \geq 3,2^{*}=\frac{2 N}{N-2}$ é o expoente crítico de Sobolev, $u_{+}=\max (u, 0), g \in C\left(\bar{\Omega} \times \mathbb{R}, \mathbb{R}^{+}\right), \lambda>\lambda_{1}, \lambda \notin \sigma(-\Delta)$ e $f \in L^{r}(\Omega), \operatorname{com} r>N$.

Com o intuito de observar as mudanças que ocorrem do caso subcrítico para o crítico e as diferentes técnicas variacionais para a resolução de problemas elípticos, estudamos, inicialmente, um problema um pouco mais antigo que $(P)$, que, por sua vez, motivou seu estudo. Tal problema é

$$
\left\{\begin{array}{lc}
-\Delta u=\lambda u+u_{+}^{p}+f, & \text { em } \Omega \\
u=0, & \text { sobre } \partial \Omega
\end{array}\right.
$$

onde consideramos o caso subcrítico, ou seja, quando $p \in\left(1,2^{*}-1\right)$.

Com o auxílio do TEOREMA DE ENLACE verificamos que tanto $(P)$ quanto $\left(P^{\prime}\right)$ têm pelo menos duas soluções fracas não triviais. 


\section{Abstract}

In this work, we aimed to study the existence of nontrivial weak solutions for the elliptic problem with critical non-linearity

$$
\begin{cases}-\Delta u=\lambda u+u_{+}^{2^{*}-1}+g\left(x, u_{+}\right)+f(x) & \text { in } \Omega \\ u=0 & \text { on } \partial \Omega\end{cases}
$$

where $\Omega$ is a bounded domain with smooth boundary in $\mathbb{R}^{N}$, with $N \geq 3,2^{*}=\frac{2 N}{N-2}$ is the critical Sobolev exponent, $u_{+}=\max (u, 0), g \in C\left(\bar{\Omega} \times \mathbb{R}, \mathbb{R}^{+}\right), \lambda>\lambda_{1}, \lambda \notin \sigma(-\Delta)$ and $f \in L^{r}(\Omega)$, with $r>N$.

In order to observe different variational techniques for solving elliptic problems, we studied initially a problem a little older than $(P)$, which, in turn, led to its study. This problem is

$$
\begin{cases}-\Delta u=\lambda u+u_{+}^{p}+f & \text { in } \Omega \\ u=0 & \text { on } \partial \Omega\end{cases}
$$

where we consider the subcritical case, that is, when $p \in\left(1,2^{*}-1\right)$.

With the aid of the LINKING THEOREM we see that both $(P)$ and $\left(P^{\prime}\right)$ have at least two nontrivial weak solutions. 


\section{Notações e Definições}

- $\Omega$ denota um domínio limitado em $\mathbb{R}^{N}$;

- $\partial \Omega$ denota a fronteira de $\Omega$;

- $\mathbb{R}_{+}^{N}=\left\{x \in \mathbb{R}^{N} ; x_{N}>0\right\}$ e $\partial \mathbb{R}_{+}^{N}=\left\{x \in \mathbb{R}^{N} ; x_{N}=0\right\}$

- Dizemos que $\Omega$ tem fronteira de classe $C^{k}$, com $k \in \mathbb{N}$, se para cada $x \in \partial \Omega$ existem $r_{x}>0$ e um difeomorfismo $\Psi_{x}: B_{x}=B\left(x, r_{x}\right) \rightarrow D \subset \mathbb{R}^{N}$ de classe $C^{k}\left(B_{x}\right)$ tais que $\Psi_{x}\left(B_{x} \cap \Omega\right) \subset \mathbb{R}_{+}^{N}$ e $\Psi_{x}\left(B_{x} \cap \partial \Omega\right) \subset \partial \mathbb{R}_{+}^{N}$. Se $\Omega$ tem fronteira $C^{k}$ para todo $k \in \mathbb{N}$, dizemos que sua fronteira é suave;

- $|\Omega|$ denota a medida de Lebesgue de $\Omega$ e $\omega_{N}$ denota a medida de Lebesgue da esfera unitária $N$-dimensional;

- Para uma função $u: \Omega \rightarrow \mathbb{R}$, definimos sua parte positiva como sendo $u(x)_{+}=$ $\max (u(x), 0)$;

- $\nabla u=\left(\frac{\partial u}{\partial x_{1}}, \ldots, \frac{\partial u}{\partial x_{N}}\right)$ denota o gradiente $u$;

- $\Delta u=\sum_{i=1}^{N} \frac{\partial^{2} u}{\partial x_{i}^{2}}$ denota o laplaciano de $u$;

- Se $X$ é um espaço normado, $\|\cdot\|_{X}$ denota a norma de $X$;

- Se $X$ é um espaço com produto interno, $\langle\cdot, \cdot\rangle_{X}$ denota o produto interno de $X$;

- Denotaremos a convergência fraca em $X$ por " $\rightarrow$ "e a convergência forte em $X$ por $" \rightarrow "$; 
- Sejam $X$ e $Y$ espaço vetroriais normados. Se $X \subset Y$, diremos que $X$ está continuamente mergulhado em $Y$ se existe $C>0$ tal que $\|x\|_{Y} \leq C\|x\|_{X}$ para todo $x \in X$. Denotamos isso por

$$
X \hookrightarrow Y
$$

Se, além disso, toda sequência limitada em $X$ admitir subsequência convergente em $Y$, diremos que $X$ está compactamente mergulhado em $Y$. Denotamos isso por

$$
X \hookrightarrow Y
$$

- Se $p \in[1, \infty), L^{p}(\Omega)=\left\{u: \Omega \rightarrow \mathbb{R}\right.$ mensurável : $\left.\int_{\Omega}|u|^{p} d x<\infty\right\}$ denota o espaço de Lebesgue com a norma dada por

$$
\|u\|_{p}=\left(\int_{\Omega}|u(x)|^{p} d x\right)^{\frac{1}{p}}
$$

- $L^{\infty}(\Omega)$ denota o espaço das funções mensuráveis que são limitadas quase toda parte (q.t.p.) em $\Omega$ com norma dada por

$$
\|u\|_{\infty}=\inf \{C>0:|u(x)| \leq C \text { para quase todo } x \in \Omega\}
$$

- $C_{0}^{\infty}(\Omega)$ denota o espaço das funções infinitamente diferenciáveis com suporte compacto em $\Omega$;

- Se $p \in[1, \infty)$ e $k \in \mathbb{N}$, definimos o espaço de Sobolev $W^{k, p}(\Omega)=\left\{u \in L^{p}(\Omega): D^{\alpha} u \in\right.$ $\left.L^{p}(\Omega), \quad \forall|\alpha| \leq k\right\}$, onde $\alpha$ é um multi-índice e $D^{\alpha} u$ é a derivada de $u$ no sentido fraco. Em $W^{k, p}(\Omega)$ consideramos a norma dada por

$$
\|u\|_{k, p}=\left(\int_{\Omega} \sum_{|\alpha| \leq k}\left|D^{\alpha} u\right|^{p} d x\right)^{\frac{1}{p}}
$$

- $W_{0}^{k, p}(\Omega)$ é o fecho do espaço $C_{0}^{\infty}(\Omega)$ em $W^{k, p}(\Omega)$ com respeito à norma $(0.1)$; 
- Consideraremos $E=W_{0}^{1,2}(\Omega)$ equipado com a norma de Dirichlet

$$
\|u\|_{E}=\left(\int_{\Omega}|\nabla u|^{2}\right)^{\frac{1}{2}}
$$

que é proveniente do produto interno

$$
\langle u, v\rangle_{E}=\int_{\Omega} \nabla u \cdot \nabla v
$$

e é equivalente à norma (0.1) para $k=1$ e $p=2$;

- Denotaremos por $0<\lambda_{1}<\lambda_{2} \leq \lambda_{3} \leq \ldots \leq \lambda_{k} \leq \ldots$ os autovalores de $-\Delta$ em $E$ e por $\sigma(-\Delta)=\left\{\lambda_{k}: k \in \mathbb{N}\right\}$ seu espectro. Denotaremos por $\left\{\phi_{k}: k \in \mathbb{N}\right\}$ as autofunções correspondentes. Lembramos que $\left\{\phi_{k}\right\}_{k \in \mathbb{N}}$ constitui um conjunto ortogonal em $E$ e em $L^{2}(\Omega)$, que $\phi_{k} \in C^{\infty}(\bar{\Omega})$ e que $\phi_{1}>0$. Podemos assumir que $\left\|\phi_{k}\right\|_{E}=1$. 


\section{Sumário}

1 Introdução $\quad 1$

2 Ferramentas básicas $\quad 5$

3 A primeira solução $\quad 9$

4 A segunda solução para o problema subcrítico $\quad 15$

4.1 Apresentação e reformulação do problema . . . . . . . . . . . . . 15

4.2 A estrutura de enlace do funcional . . . . . . . . . . . . . . . 16

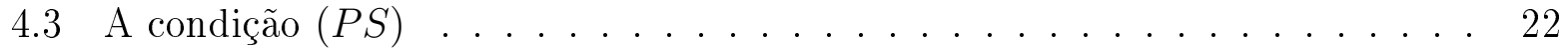

4.4 Prova do Teorema $4.1 \ldots \ldots \ldots \ldots \ldots \ldots$

5 A segunda solução para o problema crítico $\quad 27$

5.1 Apresentação do problema . . . . . . . . . . . . . . 27

5.2 Reformulação do problema . . . . . . . . . . . . . . . . . 29

5.3 Estimando a pertubação $f-f_{m} \ldots \ldots \ldots \ldots \ldots \ldots$

5.4 Funções extremais para o mergulho de Sobolev . . . . . . . . . . . . . . 33

5.5 As autofunções aproximadas . . . . . . . . . . . . . . . . . 39

5.6 Observação sobre as funções cortadas . . . . . . . . . . . . . . . . . 46

5.7 A estrutura de enlace do funcional . . . . . . . . . . . . . . 47

5.8 A solução fraca . . . . . . . . . . . . . . . . . . . . 55

5.9 Estimando os níveis de $\operatorname{minimax} \ldots \ldots \ldots \ldots$. . . . . . . . . . 61

5.10 Prova dos Teoremas 5.1 e $5.2 \ldots \ldots \ldots \ldots \ldots$

$\begin{array}{ll}\text { A Resultados Importantes } & 81\end{array}$ 
B Operador de Nemytskii

C Lema de Brezis-Lieb 


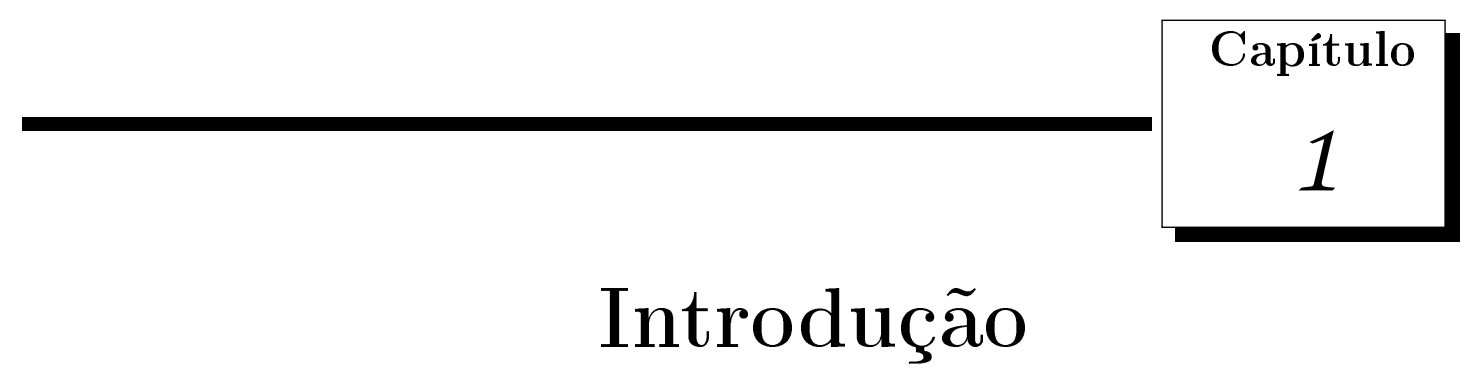

Neste trabalho tivemos como objetivo principal estudar o problema elíptico crítico

$$
\begin{cases}-\Delta u=\lambda u+u_{+}^{2^{*}-1}+g\left(x, u_{+}\right)+f(x), & \text { em } \Omega \\ u=0, & \text { sobre } \partial \Omega\end{cases}
$$

abordado por [CR02].

No entanto, estudamos inicialmente o problema elíptico subcrítico

$$
\left\{\begin{array}{lc}
-\Delta u=\lambda u+u_{+}^{p}+f(x), & \text { em } \Omega \\
u=0, & \text { sobre } \partial \Omega
\end{array}\right.
$$

abordado por [RS86].

Em todo este trabalho $\Omega \subset \mathbb{R}^{N}$ é um domínio limitado com fronteira suave, $\lambda>\lambda_{1}$, com $\lambda \notin \sigma(-\Delta)$, e $f \in L^{r}(\Omega), \operatorname{com} r>N$.

Em $(P)$ consideramos $N \geq 3,2^{*}=\frac{2 N}{N-2}$ é o expoente crítico para o mergulho de Sobolev, $g: \bar{\Omega} \times \mathbb{R} \rightarrow \mathbb{R}_{+}$é contínua, $g(\cdot, s) \equiv 0$ para todo $s \leq 0$ e $g$ tem crescimento subcrítico no infinito. Em $\left(P^{\prime}\right)$ consideramos $N \geq 2$, sendo $p \in(1, \infty)$ para $N=2$, e $p \in\left(1,2^{*}-1\right)$ para $N \geq 3$.

Os principais resultados que objetivamos provar neste trabalho são o Teorema 4.1, no capítulo 4, e os Teoremas 5.1 e 5.2, no capítulo 5. Neles afirmamos a existência de pelo menos duas soluções para os problemas $(P)$ e $\left(P^{\prime}\right)$, sob hipóteses apropriadas. 
As condições sobre $\lambda$ e $g$, nos problemas $(P)$ e $\left(P^{\prime}\right)$, implicam que a função real de variável real $k(s)=\lambda s+s_{+}^{2^{*}-1}+g\left(x, s_{+}\right)\left(\right.$ou $k(s)=\lambda s+s_{+}^{p}$ no caso do problema $\left.\left(P^{\prime}\right)\right)$ interage com todos os autovalores do laplaciano exceto os primeiros, no seguinte sentido

$$
\lambda_{1}<\lambda=\lim _{s \rightarrow-\infty} \frac{k(s)}{s}<\lim _{s \rightarrow \infty} \frac{k(s)}{s}=\infty .
$$

Tal fato, de acordo com a literatura, enquadra estes problemas no conjunto de problemas chamados de tipo Ambrosetti-Prodi, ver, por exemplo, [DFJ99].

A primeira solução fraca será sempre uma solução negativa $u_{n e g}$ comum aos problemas $(P)$ e $\left(P^{\prime}\right)$. Ela será obtida no capítulo 3 explorando o fato de a não linearidade ser sempre nula para $u$ negativas.

A segunda solução fraca será obtida através do TEOREMA DE ENLACE.

Ao estudarmos o problema $\left(P^{\prime}\right)$, veremos como construir uma geometria de enlace e como verificar a condição de Palais-Smale (condição $(P S)$ ) em um problema subcrítico. Ao estudarmos o problema $(P)$, notaremos as dificuldades adicionais de resolver um problema parecido, mas no caso crítico. Como $E=W_{0}^{1,2}(\Omega)$ não está compactamente contido em $L^{2^{*}}(\Omega)$, os argumentos variacionais clássicos não se aplicam ao funcional associado ao problema; em particular, nestas condições, o funcional não satisfaz a condição $(P S)$. Quando isso ocorre, dizemos que estamos lidando com um problema de falta de compacidade.

Esse tipo de situação foi inicialmente estudada por Brezis e Nirenberg em [BN83]. Eles consideraram o seguinte problema:

$$
\begin{cases}-\Delta u=u^{2^{*}-1}+g(x, u), & \text { em } \Omega \\ u>0, & \text { em } \Omega \\ u=0, & \text { sobre } \partial \Omega\end{cases}
$$

onde $g$ é uma pertubção de ordem inferior de $u^{2^{*}-1}$, ou seja, $\frac{g(x, s)}{s^{2 *-1}} \underset{s \rightarrow \infty}{\longrightarrow} 0$. Com hipóteses apropriadas sobre a função $g$, os autores mostraram que, mesmo trabalhando num problema onde ocorre falta de compacidade, ainda é possível encontrar uma solução fraca não trivial. Eles mostraram que em certos níveis minimax $c$ é possível obter uma sequência $(P S)$ no nível $c$ e que se $c \in\left(0, \frac{1}{N} S^{\frac{N}{2}}\right)$, onde $S$ é a melhor constante para o mergulho de Sobolev $E \hookrightarrow L^{2^{*}}(\Omega)$, então a sequência obtida admite subsequência convergente em $E$ com limite não nulo. O limite dessa subsequência é a solução fraca para o problema (1.1). 
É importante observar que se $g \equiv 0$ no problema (1.1), então não existe solução quando $\Omega$ é um conjunto estrelado, veja [Poh65]. Isso parece sugerir que a pertubação de ordem inferior $g$ exerce um papel importante para reverter a situação de falta de compacidade. Se observarmos bem, o problema $(P)$ possui uma pertubação de ordem inferior de $u^{2^{*}-1}$, logo, tomando [BN83] como fonte de inspiração, parece sugestivo esperar que seja possível contornar a falta de compacidade e obter uma solução não trivial de $(P)$.

Para obter isso, se torna importante estimar com precisão o nível minimax do funcional, para poder mostrar a existência de um ponto crítico correspondente. Para estimar este nível é, em geral, necessário explorar as propriedades das funções extremais $\Phi_{\epsilon}$ do mergulho de Sobolev. Seguindo essas ideias, de Figueiredo e Yang, em [DFJ99], provaram a existência de uma segunda solução fraca para o problema $(P)$ no caso $g \equiv 0$ e $N \geq 7$, isto é, quando a perturbação de ordem inferior é dada apenas pelo termo $\lambda u$.

No intuito de melhorar os resultados obtidos em [DFJ99], Calanchi e Ruf, em [CR02], fizeram uma abordagem diferente. A ideia, inspirada em trabalhos anteriores, por exemplo [GR97], consiste em usar oportunas funções de 'cut off', a fim de "cavar buracos" na solução negativa $u_{n e g}$ e nas autofunções do Laplaciano, e de concentrar as funções extremais $\Phi_{\epsilon}$ em bolas pequenas, de modo a obtermos funções com propriedades similares às das originais, mas com suportes disjuntos. Essas manipulações criam alguns erros, mas eles são mais fáceis de estimar que os termos mistos que surgem em expressões como $\left(v+u_{n e g}+\Phi_{\epsilon}\right)_{+}^{2^{*}}$ quando seus suportes não são disjuntos. Além disso, mover esses "pequenos buracos" para próximo da fronteira, onde a função $\left|u_{n e g}\right|$ é pequena, possibilita obter estimativas melhores que as encontradas em [DFJ99]. Com essa abordagem já é possível estender o resultado em [DFJ99] para o caso $N=6$. Para os casos $N=3,4,5$, ainda é necessário assumir que a função $g$ forneça uma ulterior perturbação de ordem inferior a $u^{2^{*}-1}$, para recuperar a compacidade.

No capítulo 2, enunciaremos alguns resultados necessários, para o estudo dos problemas citados, com as devidas referências. No capítulo 3, mostraremos como obter a primeira solução fraca, comum aos problemas $(P)$ e $\left(P^{\prime}\right)$. No capítulo 4, encontraremos a segunda solução fraca de $\left(P^{\prime}\right)$. Por fim, no capítulo 5, abordaremos o problema $(P)$, mostrando como obter sua segunda solução fraca. Buscamos evidenciar em cada caso as diferentes técnicas utilizadas que nos permitiram lançar mão dos métodos variacionais para a resolução de tais problemas. 


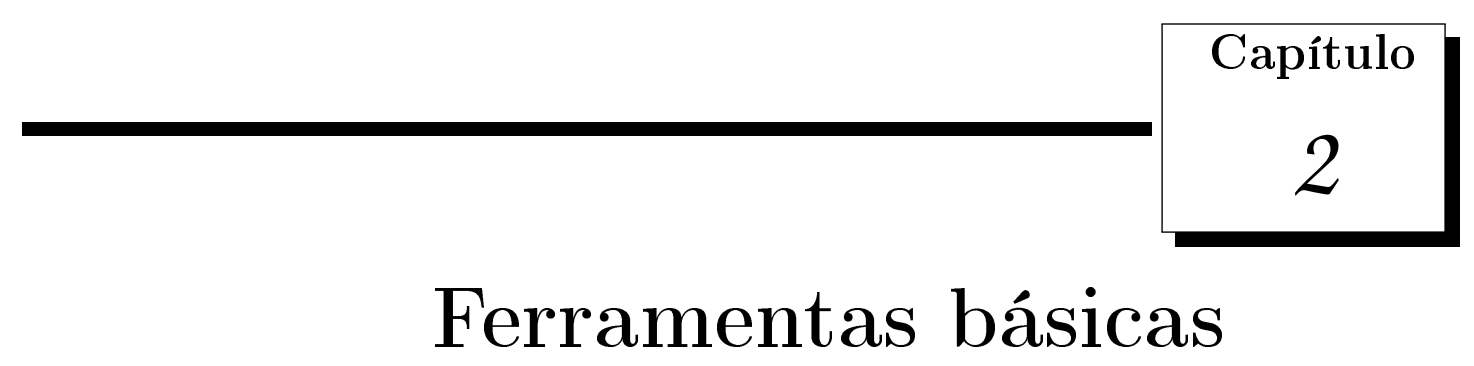

Como é rotineiro em métodos variacionais, iremos associar cada equação a um funcional $I: E \rightarrow \mathbb{R}$ definido no espaço de Banach $E$, e verificaremos se tal funcional admite algum ponto crítico não trivial; tal ponto crítico será a solução fraca não trivial que procuramos. Para esse fim enunciaremos, a seguir, algumas definições e um teorema que serão as ferramentas básicas para a procura de tais pontos críticos.

\section{Definição 2.1 (SEQUÊNCIA DE PALAIS-SMALE).}

Sejam $I \in C^{1}(E, \mathbb{R})$ e c $\in \mathbb{R}$; diremos que uma sequência $\left\{v_{n}\right\}_{n \in \mathbb{N}} \subset E$ é uma sequência de Palais-Smale para I no nível c se

$$
I\left(v_{n}\right) \underset{n \rightarrow \infty}{\longrightarrow} c \quad \text { e } \quad I^{\prime}\left(v_{n}\right) \underset{n \rightarrow \infty}{\longrightarrow} 0 .
$$

Nos referiremos a tais sequências apenas como sequências $(P S)$ para I no nivel c.

\section{Definição 2.2 (CONDIÇÃO DE PALAIS-SMALE).}

Sejam $I \in C^{1}(E, \mathbb{R})$ e $c \in \mathbb{R}$; diremos que o funcional I satisfaz a condição de PalaisSmale no nivel c se qualquer sequência $(P S)$ para I no nível c admite uma subsequência convergente. Nos referiremos a tal condição como condição $(P S)_{c}$. Além disso, diremos que o funcional I satisfaz a condição de Palais-Smale (condição (PS)) se I satisfaz a condição $(P S)_{c}$ para todo $c \in \mathbb{R}$.

Definição 2.3 (ENLACE).

Seja $S$ um subconjunto fechado de E. Seja $Q$ um subconjunto de E homeomorfo à bola 
unitária fechada $\bar{B}_{1}(0)$ de $\mathbb{R}^{k}$ para algum natural $k \geq 2$. Denote por $\partial Q$ o subconjunto de $Q$ correspondente à fronteira de $\bar{B}_{1}(0)$ segundo o homeomorfismo. Dizemos que $S$ e $Q$ enlaçam se

(a) $S \cap \partial Q=\emptyset$;

(b) dado $h \in C(E, E)$, com $\left.h\right|_{\partial Q}=I d$, vale $h(Q) \cap S \neq \emptyset$.

\section{Exemplo 2.4.}

Considere $E=W \oplus X$, com $\operatorname{dim} W=k-1$, onde $k \geq 2$. Sejam $B_{\rho}=\left\{u \in E:\|u\|_{E}<\rho\right\}$, onde $\rho>0$, e e $\in X$, com $\|e\|_{E}=1$. Sejam $r>0$ e $R>\rho$; defina os subconjuntos

$$
S_{\rho}=\partial B_{\rho} \cap X \quad e \quad Q_{R, r}=\left(\bar{B}_{r} \cap W\right) \oplus\{s e: s \in[0, R]\} .
$$

É possivel mostrar que $Q_{R, r}$ é homeomorfo à bola unitária fechada de $\mathbb{R}^{k}$. Além disso, o exemplo 8.3 de [Str08] mostra que esses conjuntos enlaçam. Neste caso, temos que $\partial Q_{R, r}=$ $\Gamma_{1} \cup \Gamma_{2} \cup \Gamma_{3}$ onde

$$
\begin{aligned}
& \Gamma_{1}=\left\{u \in E: u \in W, \quad\|u\|_{E} \leq r\right\}, \\
& \Gamma_{2}=\left\{u \in E: u=w+s e, \quad w \in W, \quad\|w\|_{E}=r, \quad s \in(0, R)\right\}, \\
& \Gamma_{3}=\left\{u \in E: u=w+R e, \quad w \in W, \quad\|w\|_{E} \leq r\right\} .
\end{aligned}
$$

Durante a prova da existência da segunda solução fraca para os problemas $(P)$ e $\left(P^{\prime}\right)$ necessitaremos construir conjuntos como em (2.1).

O teorema a seguir é de fundamental importância para o desenvolvimento deste trabalho. Ele pode ser visto como um caso particular do Teorema 4.3, combinado com os corolários 4.2 e 4.3 de [MW89].

\section{Teorema 2.5 (TEOREMA DE ENLACE).}

Seja $I \in C^{1}(E, \mathbb{R})$ e sejam $S$ e $Q$ subconjuntos de $E$ que enlaçam, como na Definição 2.3. Suponha que existam duas constantes $\beta<\alpha$ tais que

a) $\left.I\right|_{S} \geq \alpha$;

b) $\left.I\right|_{\partial Q} \leq \beta$. 
Então existe uma sequência $(P S)$ para I no nível $c$ onde $c \geq \alpha$; além disso, $c$ pode ser caracterizado como $c=\inf _{h \in \Gamma} \max _{u \in Q} I(h(u))$, onde

$$
\Gamma=\left\{h \in C(E, E):\left.h\right|_{\partial Q}=I d\right\}
$$

Observe que, uma vez obtida a sequência $(P S)$ fornecida pelo TEOREMA DE ENLACE, caso o funcional satisfaça a condição $(P S)$ ou $(P S)_{c}$, já teremos obtido a existência de um ponto crítico no nível $c$. Em caso contrário, ainda faltará mostrar que é possível obter alguma subsequência convergente. 


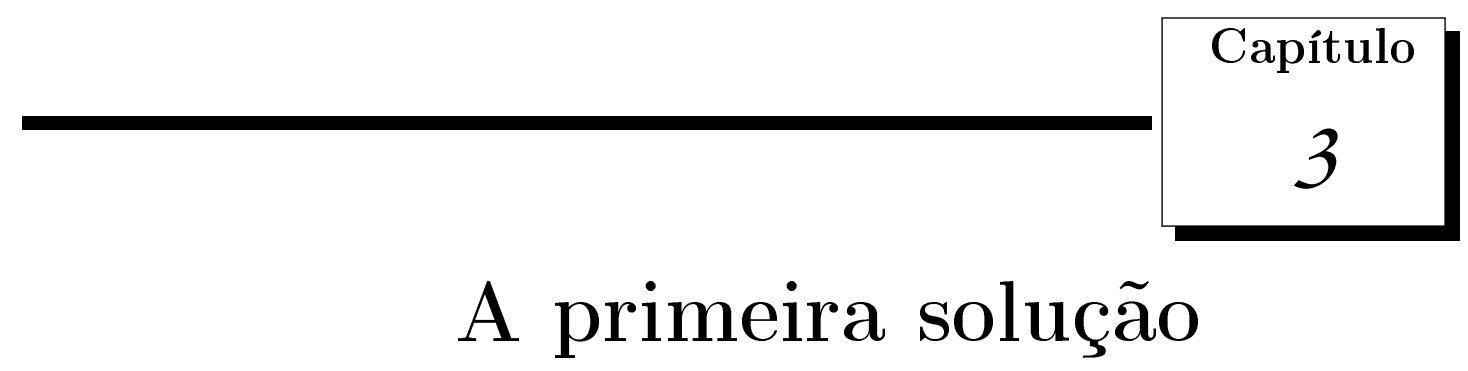

Neste capítulo mostraremos como obter a primeira solução fraca comum aos problemas $(P)$ e $\left(P^{\prime}\right)$. Para isso, consideraremos o problema mais geral

$$
\left\{\begin{array}{lc}
-\Delta u=\lambda u+u_{+}^{p}+g\left(x, u_{+}\right)+f(x), & \text { em } \Omega \\
u=0, & \text { sobre } \partial \Omega
\end{array}\right.
$$

onde $N \geq 2$, sendo $p \in\left(1,2^{*}\right]$ se $N \geq 3$ e $p \in(1, \infty)$ se $N=2$; além disso, $\Omega \subset \mathbb{R}^{N}$ é um domínio limitado com fronteira suave, $g: \bar{\Omega} \times \mathbb{R} \rightarrow \mathbb{R}_{+}$é uma função contínua tal que $g(\cdot, s) \equiv 0$ para todo $s \leq 0, \lambda>\lambda_{1}, \operatorname{com} \lambda \notin \sigma(-\Delta)$ e $f \in L^{r}(\Omega), \operatorname{com} r>N$.

Daqui em diante consideraremos

$$
f=h+t \phi_{1}
$$

onde $t \in \mathbb{R}, \phi_{1}>0$ é a primeira autofunção do Laplaciano em $E$ e $h \in L^{r}(\Omega)$.

\section{Teorema 3.1.}

Dado $h \in L^{r}(\Omega)$ existe $T=T(h)$ tal que para todo $t>T$ o problema $(\widetilde{P})$ admite uma solução fraca negativa $u_{t} \in E=W_{0}^{1,2}$ tal que $u_{t} \in W_{0}^{1, r}(\Omega) \cap W^{2,2}(\Omega)$. Além disso, $u_{t} \in C^{1,1-\frac{N}{r}}(\bar{\Omega})$.

\section{Prova.}

Como $2 \leq N<r$, temos que $h \in L^{2}(\Omega)$. Para obtermos o resultado desejado, resolveremos, então, os seguintes problemas auxiliares. 
1. Dado $h \in L^{2}(\Omega)$, considere

$$
\begin{cases}-\Delta u=\lambda u+h, & \text { em } \quad \Omega \\ u=0, & \text { sobre } \partial \Omega\end{cases}
$$

2. Dado $t \in \mathbb{R}$, considere

$$
\begin{cases}-\Delta w=\lambda w+t \phi_{1}, & \text { em } \quad \Omega \\ w=0, & \text { sobre } \partial \Omega .\end{cases}
$$

Mostraremos que existe uma única solução para (3.2) e para (3.3), neste último caso para $t$ fixo.

Comecemos com o problema (3.2). Para resolvê-lo, consideraremos primeiramente o seguinte problema

$$
\begin{cases}-\Delta u=h, & \text { em } \Omega \\ u=0, & \text { sobre } \partial \Omega .\end{cases}
$$

Definamos as aplicações $A: E \times E \rightarrow \mathbb{R}$ e $H: E \rightarrow \mathbb{R}$ respectivamente por

$$
A(u, v)=\int_{\Omega} \nabla u \cdot \nabla v d x \quad \text { e } \quad H(v)=\int_{\Omega} h v d x .
$$

Utilizando a desigualdade de Hölder observamos facilmente que $A$ é uma forma bilinear contínua e coerciva. Também é fácil observar que $H \in E^{\prime}$, para isso utilizamos a desigualdade de Hölder junto com o item (i) do TEOREMA DE MERGULHO DE SOBOLEV (Teorema A.2). Logo, pelo TEOREMA DE LAX - MILGRAN (Teorema A.5), existe um único $u \in E$ tal que $A(u, v)=H(v)$ para todo $v \in E$, ou seja, existe um único $u \in E$ que

$$
\int_{\Omega} \nabla u \cdot \nabla v d x=\int_{\Omega} h v d x \quad \forall v \in E .
$$

Isso implica que $u$ é a única solução fraca de (3.4). Podemos então definir o operador linear $\widetilde{T}: L^{2}(\Omega) \rightarrow E$ que associa cada $h \in L^{2}(\Omega)$ à única solução fraca $u=\widetilde{T}(h) \in E$ de $(3.4)$.

Fazendo $u=v$ em (3.6) e usando mais uma vez a desigualdade de Hölder e o item (i) do 
TEOREMA DE MERGULHO DE SOBOLEV, segue que

$$
\begin{aligned}
\|u\|_{E}^{2} & =\int_{\Omega}|\nabla u|^{2} d x \\
& =\int_{\Omega} h u d x \\
& \leq\|h\|_{2}\|u\|_{2} \\
& \leq C\|h\|_{2}\|u\|_{E}
\end{aligned}
$$

logo, se $u \in E \backslash\{0\}$, então $\|\widetilde{T}(h)\|_{E}=\|u\|_{E} \leq C\|h\|_{2}<\infty$ e isso nos mostra que $\widetilde{T}$ é contínuo. Além disso, como a imersão $I: E \rightarrow L^{2}(\Omega)$ é um operador compacto, temos que o operador $T: L^{2}(\Omega) \rightarrow L^{2}(\Omega)$ dado por

$$
T(h)=(I \circ \widetilde{T})(h)
$$

também o é.

Pela passagem anterior, temos que $u_{0} \in E$ será uma solução fraca de (3.2) se, e só se, $u_{0}=T\left(\lambda u_{0}+h\right)$. No entanto, nos perguntamos se dado $h \in L^{2}(\Omega)$ existe $u_{0} \in E$ tal que $u_{0}=T\left(\lambda u_{0}+h\right)$. Caso exista, $u_{0}$ é único? Se a resposta a uma dessas perguntas for negativa, não poderemos chegar à conclusão que queremos. A afirmação a seguir nos dá a resposta desejada:

\section{AFIRMAÇÃO.}

Dado $h \in L^{2}(\Omega)$ existe um único $u_{h} \in$ E satisfazendo

$$
u_{h}=T\left(\lambda u_{h}+h\right)
$$

\section{Prova da AFIRMAÇÃO.}

Como $T$ é um operador compacto, pelo TEOREMA DA ALTERNATIVA DE FREDHOLM (Teorema A.4), temos que a aplicação $I d-\lambda T: L^{2}(\Omega) \rightarrow L^{2}(\Omega)$ é inversível ou tem núcleo não trivial. Se o núcleo da aplicação $I d-\lambda T$ for não trivial, então existe $w \in L^{2}(\Omega) \backslash\{0\}$ 
tal que $\lambda T(w)=w$, equivalentemente $w$ é a única solução fraca de (3.4), ou seja,

$$
\begin{cases}-\Delta w=\lambda w, & \text { em } \Omega \\ w=0, & \text { sobre } \partial \Omega .\end{cases}
$$

Mas isso contraria a hipótese de $\lambda \notin \sigma(-\Delta)$.

Concluímos, então, que $I d-\lambda T$ é inversível; sendo assim, dado $h \in L^{2}(\Omega)$, existe um único $u_{h} \in L^{2}(\Omega)$ tal que

$$
(I d-\lambda T)\left(u_{h}\right)=T(h)
$$

equivalentemente

$$
u_{h}=T\left(\lambda u_{h}+h\right)
$$

Pela definição de $T, u_{h} \in E$; assim fica provada a afirmação.

Da afirmação, segue que $u_{0} \in E$ é de fato a única solução fraca de (3.2) para $h \in L^{2}(\Omega)$ fixo.

Como $h \in L^{r}(\Omega)$, temos, pelo TEOREMA DE REGULARIDADE (Teorema A.6), que $u_{0} \in W_{0}^{1, r}(\Omega) \cap W^{2, r}(\Omega)$. Do item iii do TEOREMA DE MERGULHO DE SOBOLEV, com $k=2$, segue que $u_{0} \in C^{1, \beta}(\bar{\Omega})$, onde $0<\beta \leq 1-\frac{N}{r}$; em particular $u_{0} \in C^{1,1-\frac{N}{r}}(\bar{\Omega})$. Logo, $u_{0}$ e suas derivadas de primeira ordem são limitadas em $\bar{\Omega}$.

Como $\phi_{1} \in C^{\infty}(\bar{\Omega})$, não é difícil verificar que $w_{t}=\frac{t}{\lambda_{1}-\lambda} \phi_{1}$ é a única solução fraca de (3.3) para cada $t \in \mathbb{R}$.

Considere $u_{t}=u_{0}+w_{t}$. Pelas propriedades de $u_{0}$ e $\phi_{1}$, temos que $u_{t} \in W_{0}^{1, r}(\Omega) \cap W^{2, r}(\Omega)$ e $u_{t} \in C^{1,1-\frac{N}{r}}(\Omega)$. Além disso, é simples verificar que o fato de $u_{0}$ ser solução de $(3.2)$ e $w_{t}$ de (3.3) nos dá

$$
-\Delta u_{t}-\lambda u_{t}=f
$$

Observe agora que se pudermos afirmar que $u_{t} \leq 0$ em $\Omega$ para algum $t \in \mathbb{R}$, então podemos dizer que $(3.7)$ é equivalente a $(\widetilde{P})$ uma vez que, neste caso, $\left(u_{t}\right)_{+} \equiv g\left(\cdot,\left(u_{t}\right)_{+}\right) \equiv 0$. Devemos então verificar se existem valores de $t \in \mathbb{R}$ para os quais vale $u_{t} \leq 0$, ou seja, 
devemos verificar se é possível termos a seguinte desigualdade

$$
\frac{u_{0}(x)}{\phi_{1}(x)} \leq \frac{t}{\lambda-\lambda_{1}} \quad \forall x \in \Omega
$$

para algum $t \in \mathbb{R}$.

Como $\phi_{1}>0$ em $\Omega$, está bem definida e é contínua a função $U: \Omega \rightarrow \mathbb{R}$ dada por

$$
U(x)=\frac{u_{0}(x)}{\phi_{1}(x)}
$$

Queremos mostrar agora que $U$ é limitada em $\Omega$. Suponhamos então que $U$ não seja limitada em $\Omega$; logo, existem $\left\{x_{n}\right\}_{\mathbb{N}} \subset \Omega$ e $\left\{c_{n}\right\}_{\mathbb{N}} \subset \mathbb{R}$ tais que $\left|u_{0}\left(x_{n}\right)\right|=c_{n} \phi_{1}\left(x_{n}\right)$ com $c_{n} \underset{n \rightarrow \infty}{\longrightarrow} \infty$. Observe que, a menos de subsequência, $x_{n} \underset{n \rightarrow \infty}{\longrightarrow} x_{0}$ para algum $x_{0} \in \partial \Omega$, pois, em qualquer compacto contido em $\Omega$, temos $\phi>\delta>0$ e $u_{0}$ é limitada.

Para cada $n \in \mathbb{N}$ seja $y_{n} \in \partial \Omega$ tal que $\left\|x_{n}-y_{n}\right\|=\operatorname{dist}\left(x_{n}, \partial \Omega\right)$. Note que $y_{n} \underset{n \rightarrow \infty}{\longrightarrow} x_{0}$. Como $\Omega$ tem fronteira suave, temos que o vetor $y_{n}-x_{n}$ é uma normal exterior a $\partial \Omega$ em $y_{n}$, logo o vetor $\frac{y_{n}-x_{n}}{\left\|y_{n}-x_{n}\right\|}$ converge ao vetor $\vec{n}$ que é a normal exterior unitária a $\partial \Omega$ em $x_{0}$.

Como $u_{0} \in C^{1}(\bar{\Omega})$, temos que $\max _{x \in \bar{\Omega}}\left|\nabla u_{0}(x)\right| \leq C$, onde $C>0$, logo

$$
\begin{aligned}
\frac{\phi_{1}\left(x_{n}\right)-\phi_{1}\left(y_{n}\right)}{\left\|x_{n}-y_{n}\right\|} & =\frac{\phi_{1}\left(x_{n}\right)}{\left\|x_{n}-y_{n}\right\|}=\frac{\left|u_{0}\left(x_{n}\right)\right|}{c_{n}\left\|x_{n}-y_{n}\right\|} \\
& \leq \frac{C\left\|x_{n}-y_{n}\right\|}{c_{n}\left\|x_{n}-y_{n}\right\|}=\frac{C}{c_{n}} \underset{n \rightarrow \infty}{\longrightarrow} 0 .
\end{aligned}
$$

Por outro lado, como $\phi_{1} \in C^{1}(\bar{\Omega})$, existem $\xi_{n} \in[0,1]$ tais que

$$
\lim _{n \rightarrow \infty} \frac{\phi_{1}\left(x_{n}\right)-\phi_{1}\left(y_{n}\right)}{\left\|x_{n}-y_{n}\right\|}=\lim _{n \rightarrow \infty} \frac{\nabla \phi_{1}\left(\xi_{n} x_{n}+\left(1-\xi_{n}\right) y_{n}\right) \cdot\left(x_{n}-y_{n}\right)}{\left\|x_{n}-y_{n}\right\|}=-\nabla \phi_{1}\left(x_{0}\right) \cdot \vec{n}
$$

de (3.9) e de (3.10) segue que $\nabla \phi_{1}\left(x_{0}\right) \cdot \vec{n}=0$, mas isso contraria o Lema 3.4 de [GT01].

Portanto, $U$ deve ser limitada em $\Omega$.

Podemos então considerar $T:=T(h)=\left(\lambda-\lambda_{1}\right) \max _{x \in \Omega}\left\{\frac{u_{0}(x)}{\phi_{1}(x)}\right\}$ e observar que se tomarmos qualquer $t>T$, obtemos (3.8) o que implica $u_{t}<0 \mathrm{em} \Omega$.

A função $u_{t}$ é, portanto, a solução negativa de $(\widetilde{P})$ que procurávamos. 


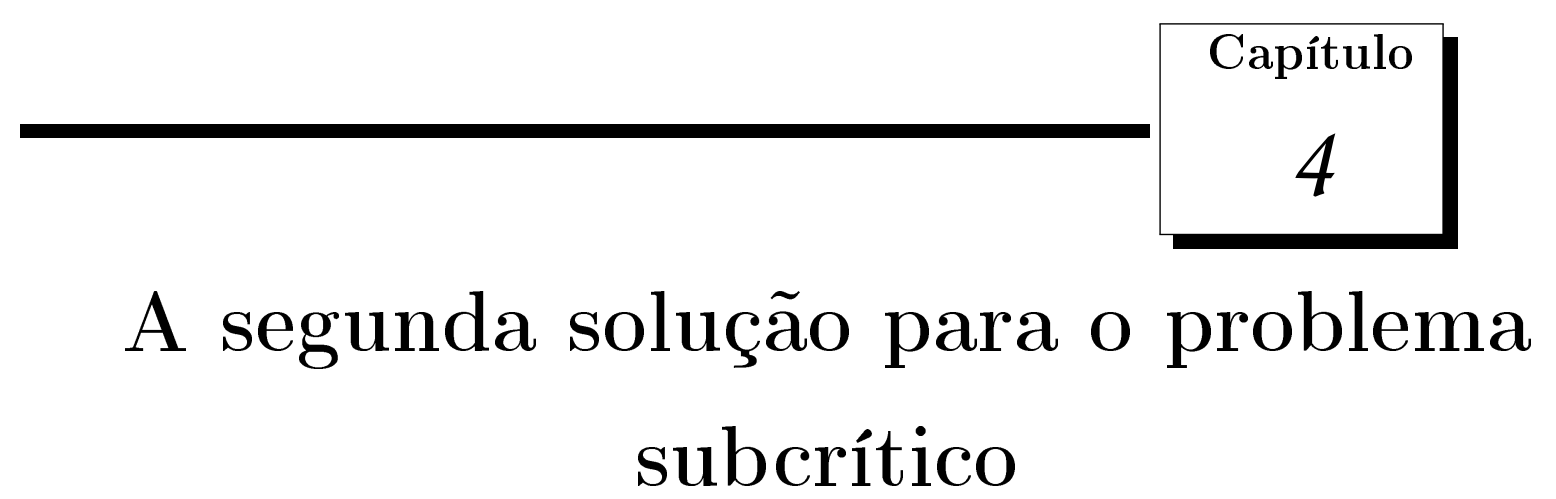

\subsection{Apresentação e reformulação do problema}

Estudamos neste capítulo o problema elíptico subcrítico abordado em [RS86].

Seja $\Omega \subset \mathbb{R}^{N}(N \geq 2)$ um domínio limitado com fronteira suave. Consideraremos o problema elípitico superlinear com condição de fronteira de Dirichlet dado por

$$
\begin{cases}-\Delta u=\lambda u+u_{+}^{p}+f(x), & \text { em } \Omega \\ u=0, & \text { sobre } \partial \Omega .\end{cases}
$$

onde $p \in\left(1,2^{*}-1\right)$, se $N \geq 3$, e $p \in(1, \infty)$, se $N=2 ; \lambda>\lambda_{1}$ é tal que $\lambda \notin \sigma(-\Delta)$ e $f=h+t \phi_{1}$ onde $h \in L^{r}(\Omega)$, com $r>N$ como em (3.1).

Observe que a função real de variável real $k(s)=\lambda s+s_{+}^{p}$ interage com todos os autovalores do Laplaciano exceto os primeiros no seguinte sentido

$$
\lambda_{1}<\lambda=\lim _{s \rightarrow-\infty} \frac{k(s)}{s}<\lim _{s \rightarrow \infty} \frac{k(s)}{s}=\infty
$$

tal fato, de acordo com a literatura, enquadra o problema $(P)$ no conjunto de problemas chamados de tipo Ambrosetti-Prodi, ver, por exemplo, [DFJ99].

Objetivamos provar o seguinte teorema: 
Teorema 4.1.

Se $\lambda>\lambda_{1}$ e $\lambda \notin \sigma(-\Delta)$, então dada $h \in L^{r}(\Omega)$ existe uma constante $T=T(h)$ tal que para $t>T$ o problema $\left(P^{\prime}\right)$ admite pelo menos duas soluções fracas.

A primeira solução fraca é a solução negativa $u_{t}$ obtida do Teorema 3.1, onde $t>T(h)$ é dado.

Para encontrarmos uma segunda solução fraca de $\left(P^{\prime}\right)$, consideraremos $u=v+u_{t}$ e o problema

$$
\begin{cases}-\Delta v=\lambda v+\left(v+u_{t}\right)_{+}^{p}, & \text { em } \quad \Omega \\ v=0, & \text { sobre } \partial \Omega\end{cases}
$$

Observe que $u$ será solução fraca de $\left(P^{\prime}\right)$ se e só se $v$ for solução fraca de $\left(P_{t}^{\prime}\right)$, de fato

$$
\begin{aligned}
-\Delta u=\lambda u+u_{+}^{p}+f & \Longleftrightarrow-\Delta v-\Delta u_{t}=\lambda v+\left(v+u_{t}\right)_{+}^{p}+\lambda u_{t}+f \\
& \Longleftrightarrow-\Delta v=\lambda v+\left(v+u_{t}\right)_{+}^{p} .
\end{aligned}
$$

Observe também que $v=0$ é uma solução fraca trivial para $\left(P_{t}^{\prime}\right)$ correspondente à solução negativa $u_{t}$ de $\left(P^{\prime}\right)$. Logo, encontrar uma segunda solução fraca de $\left(P^{\prime}\right)$ é equivalente a encontrar uma solução fraca não trivial de $\left(P_{t}^{\prime}\right)$.

Nosso objetivo agora é procurar pontos críticos não nulos do funcional $I \in C^{1}(E, \mathbb{R})$ dado por

$$
I(v)=\frac{1}{2} \int_{\Omega}\left(|\nabla v|^{2}-\lambda v^{2}\right) d x-\frac{1}{p+1} \int_{\Omega}\left(v+u_{t}\right)_{+}^{p+1} d x
$$

cuja derivada é dada por

$$
I^{\prime}(v) \cdot \varphi=\int_{\Omega} \nabla v \cdot \nabla \varphi d x-\lambda \int_{\Omega} v \varphi d x-\int_{\Omega}\left(v+u_{t}\right)_{+}^{p} \varphi d x \quad \forall \varphi \in E .
$$

Um tal ponto crítico, se existir, será uma solução fraca não trivial de $\left(P_{t}^{\prime}\right)$.

\subsection{A estrutura de enlace do funcional}

De acordo com o exposto no capítulo 2, uma forma de procurarmos pontos críticos do funcional $I$ dado por (4.1) é verificar se $I$ satisfaz a condição $(P S)$ ou $(P S)_{c}$ e verificar se I satisfaz as hipóteses do TEOREMA DE ENLACE. 
Nesta seção mostraremos que $I$ tem estrutura de enlace, ou seja, mostraremos que existem subconjuntos fechados $S$ e $Q$ de $E$ que enlaçam e constantes $\beta<\alpha$ para os quais são válidas as hipóteses do TEOREMA DE ENLACE para o funcional $I$. Para isso, recordamos que $\lambda_{1}<\lambda$ e que $\lambda \notin \sigma(-\Delta)$, logo deve existir um natural $k \geq 1$ tal que

$$
\lambda_{k}<\lambda<\lambda_{k+1}
$$

Considere, então, $W_{k}:=\left\langle\phi_{i}\right\rangle_{i=1}^{k}$ e $X_{k}:=W_{k}^{\perp}$, ou seja, $W_{k}$ é o subespaço de $E$ gerado pelas $k$ primeiras autofunções do Laplaciano e $X_{k}$ seu complemento ortogonal em $E$. Observe que desse modo temos $E=W_{k} \oplus X_{k}$.

Os lemas a seguir são ferramentas essenciais para mostrar que o funcional $I$, de fato, tem estrutura de enlace.

\section{Lema 4.2.}

Dado $\varepsilon>0$, existe $e \in X_{k}$, com $\|e\|_{E}=\varepsilon$, tal que o conjunto

$$
\left\{x \in \Omega ; w(x)+e(x)>1, \forall w \in W_{k},\|w\|_{E} \leq 1\right\}
$$

tem medida positiva.

\section{Prova.}

Observe que se $w \in W_{k}$, então $w \in L^{\infty}(\Omega)$ uma vez que $\phi_{i} \in C^{\infty}(\bar{\Omega})$ e $w=\sum_{i=1}^{k} w_{i} \phi_{i}$. Além disso, como $W_{k}$ é um espaço de dimensão finita, todas as normas são equivalentes; logo existe $M>0$ tal que $\|w\|_{\infty} \leq M$ para todo $w \in W_{k}$ com $\|w\|_{E} \leq 1$.

Pela observação A.3 no apêndice A, podemos dizer que $X_{k} \not \subset L^{\infty}(\Omega)$; logo existe $e \in X_{k}$, cuja norma em $E$ pode ser considerada igual a $\varepsilon$, tal que $\|e\|_{\infty}=\infty$. Isso implica que dado $C>0$, existe $A_{C} \subset \Omega$, com $\left|A_{C}\right|>0$, tal que $\left.e\right|_{A_{C}}>C$. Em particular, considerando $C=M+1$, podemos observar que em $A_{M+1}$

$$
e+w>M+1-M=1 \quad \text { para todo } w \in W_{k}, \operatorname{com}\|w\|_{E} \leq 1 .
$$

Isso prova o lema.

\section{Lema 4.3.}

Seja $e \in X_{k}$ a função obtida no Lema 4.2. Se $f \in C(\bar{\Omega})$ é uma função negativa e $q \in$ 
$\left[1,2^{*}-1\right)$ é dado, então existem $\eta, r_{0}>0$ tais que

$$
\int_{\Omega}\left(w+e+\frac{f}{r}\right)_{+}^{q+1} d x \geq \eta>0
$$

para todo $w \in W_{k}$ com $\|w\|_{E} \leq 1$ e todo $r \geq r_{0}$.

\section{Prova.}

Pelo Lema 4.2, o conjunto

$$
A_{e}=\left\{x \in \Omega: w(x)+e(x)>1, \forall w \in W_{k},\|w\|_{E} \leq 1\right\}
$$

tem medida positiva, ou seja, existe $\eta>0$ tal que $\left|A_{e}\right| \geq 2^{q+1} \eta>0$. Note que $\eta$ depende apenas de $e$.

Por hipótese, existe $K>0$ tal que $-K \leq f \leq 0$. Daí, por um lado temos que para qualquer $r>0$

$$
\int_{\Omega}\left(w+e+\frac{f}{r}\right)_{+}^{q+1} d x \leq \int_{\Omega}(w+e)_{+}^{q+1} d x<\infty .
$$

Por outro lado, se considerarmos $r_{0}>2 K$, sobre o conjunto $A_{e}$ obteremos

$$
w+e+\frac{f}{r_{0}} \geq 1+\frac{f}{r_{0}}>1-\frac{K}{r_{0}}>\frac{1}{2},
$$

assim temos

$$
\int_{\Omega}\left(w+e+\frac{f}{r_{0}}\right)_{+}^{q+1} d x \geq \int_{A_{e}}\left(w+e+\frac{f}{r_{0}}\right)_{+}^{q+1} d x \geq \frac{1}{2^{q+1}}\left|A_{e}\right| \geq \eta>0 .
$$

Como para todo $r \geq r_{0}$ temos $\left(w+e+\frac{f}{r_{0}}\right)_{+} \leq\left(w+e+\frac{f}{r}\right)_{+}$, o lema está provado.

Com o auxílio da Proposição A.1 e dos Lemas 4.2 e 4.3, mostraremos que as hipóteses do TEOREMA DE ENLACE valem para nosso funcional $I$.

Recordemos que dado $\rho>0, B_{\rho}=\{v \in E:\|v\|<\rho\}$.

\section{Lema 4.4.}

Existem $\rho, \alpha>0$ tais que se $S_{\rho}=\partial B_{\rho} \cap X_{k}$, então

$$
\left.I\right|_{S_{\rho}} \geq \alpha
$$




\section{Prova.}

Queremos mostrar que existem constantes $\rho, \alpha>0$ tais que para todo $v \in S_{\rho}$ vale $I(v) \geq \alpha$. De fato, se $v \in S_{\rho}$, usando o item (b) da Proposição A.1 e o item (i) do TEOREMA DE MERGULHO DE SOBOLEV, obtemos

$$
\begin{aligned}
I(v) & =\frac{1}{2} \int_{\Omega}|\nabla v|^{2} d x-\frac{\lambda}{2} \int_{\Omega} v^{2} d x-\frac{1}{p+1} \int_{\Omega}\left(v+u_{t}\right)_{+}^{p+1} d x \\
& \geq \frac{1}{2} \int_{\Omega}|\nabla v|^{2} d x-\frac{\lambda}{2 \lambda_{k+1}} \int_{\Omega}|\nabla v|^{2} d x-\frac{1}{p+1} \int_{\Omega}(v)_{+}^{p+1} d x \\
& \geq \frac{1}{2}\left(1-\frac{\lambda}{\lambda_{k+1}}\right) \int_{\Omega}|\nabla v|^{2} d x-\frac{1}{p+1} \int_{\Omega}|v|^{p+1} d x \\
& \geq \frac{1}{2}\left(1-\frac{\lambda}{\lambda_{k+1}}\right)\|v\|_{E}^{2}-C\|v\|_{E}^{p+1} \\
& =\left[\frac{1}{2}\left(1-\frac{\lambda}{\lambda_{k+1}}\right)-C\|v\|_{E}^{p-1}\right]\|v\|_{E}^{2}
\end{aligned}
$$

Como $\lambda \in\left(\lambda_{k}, \lambda_{k+1}\right)$, podemos considerar

$$
\|v\|_{E}=\rho=\left[\frac{1}{4 C}\left(1-\frac{\lambda}{\lambda_{k+1}}\right)\right]^{\frac{1}{p-1}} \quad \text { e } \quad \alpha=\left[\frac{1}{4}\left(1-\frac{\lambda}{\lambda_{k+1}}\right)\right]^{\frac{p+1}{p-1}}\left(\frac{1}{C}\right)^{\frac{2}{p-1}}
$$

e obtermos o resultado que queríamos.

\section{Lema 4.5.}

Existem $\epsilon>0, R>\frac{\rho}{\epsilon}$ e $\widetilde{e} \in X_{k}$, com $\|\widetilde{e}\|_{E}=1$, tais que se

$$
Q_{R, \epsilon}=\left(\bar{B}_{R} \cap W_{k}\right) \oplus\{s \widetilde{e}: s \in[0, \epsilon R]\},
$$

então

$$
\left.I\right|_{\partial Q_{R, \epsilon}} \leq 0
$$

\section{Prova.}

Escolha $\epsilon>0$ de modo que $\epsilon^{2}<\frac{\lambda}{\lambda_{k}}-1$. Seja $e \in X_{k}$ a função obtida do Lema 4.2 tal que $\|e\|_{E}=\epsilon$ e considere $\widetilde{e}=\frac{e}{\epsilon}$.

Queremos mostrar que existe $R>\frac{\rho}{\epsilon}$ tal que se $v \in \partial Q_{R, \epsilon}$, então $I(v) \leq 0$.

Observe que $Q_{R, \epsilon}$ é homeomorfo à esfera unitária fechada de $\mathbb{R}^{k+1}$; logo, de acordo com 
o exemplo 2.4, temos que $\partial Q_{R, \epsilon}=\cup_{i=1}^{3} \Gamma_{i}$ onde

$$
\begin{aligned}
& \Gamma_{1}=\left\{v \in E: v \in W_{k}, \quad\|v\|_{E} \leq R\right\}, \\
& \Gamma_{2}=\left\{v \in E: v=w+s \widetilde{e}, \quad w \in W_{k}, \quad\|w\|_{E}=R, \quad s \in(0, \epsilon R)\right\}, \\
& \Gamma_{3}=\left\{v \in E: v=w+\epsilon R \widetilde{e}, \quad w \in W_{k} \quad\|w\|_{E} \leq R\right\} .
\end{aligned}
$$

Temos então que:

- Se $v \in \Gamma_{1}$, usando o item (a) da Proposição A.1, temos

$$
\begin{aligned}
I(v) & =\frac{1}{2} \int_{\Omega}|\nabla v|^{2} d x-\frac{\lambda}{2} \int_{\Omega}|v|^{2} d x-\frac{1}{p+1} \int_{\Omega}\left(v+u_{t}\right)_{+}^{p+1} d x \\
& \leq \frac{1}{2} \int_{\Omega}|\nabla v|^{2} d x-\frac{\lambda}{2} \int_{\Omega}|v|^{2} d x \\
& \leq \frac{1}{2}\left(\lambda_{k}-\lambda\right) \int_{\Omega}|v|^{2} d x ;
\end{aligned}
$$

como $\lambda_{k}<\lambda$, temos que

$$
I(v) \leq 0 \quad \forall v \in \Gamma_{1} .
$$

- Se $v=w+s \widetilde{e} \in \Gamma_{2}$, dado $R>0$ temos

$$
\begin{aligned}
I(v)= & I(w+s \widetilde{e}) \\
= & \frac{1}{2} \int_{\Omega}|\nabla(w+s \widetilde{e})|^{2} d x-\frac{\lambda}{2} \int_{\Omega}|w+s \widetilde{e}|^{2} d x \\
& \quad-\frac{1}{p+1} \int_{\Omega}\left(w+s \widetilde{e}+u_{t}\right)_{+}^{p+1} d x \\
\leq & \frac{1}{2}\|w+s \widetilde{e}\|_{E}^{2}-\frac{\lambda}{2} \| w+\left.s \widetilde{e}\right|_{2} ^{2} .
\end{aligned}
$$

Como $w$ e $\widetilde{e}$ são funções ortogonais tanto em $E$ quanto em $L^{2}(\Omega)$, temos

$$
\begin{aligned}
I(v) & \leq \frac{1}{2}\|w\|_{E}^{2}-\frac{\lambda}{2}\|w\|_{2}^{2}+\frac{1}{2} s^{2}\|\widetilde{e}\|_{E}^{2}-\frac{\lambda}{2} s^{2}\|\widetilde{e}\|_{2}^{2} \\
& \leq \frac{1}{2}\|w\|_{E}^{2}-\frac{\lambda}{2 \lambda_{k}}\|w\|_{E}^{2}+\frac{1}{2} \epsilon^{2} R^{2}\|\widetilde{e}\|_{E}^{2}
\end{aligned}
$$




$$
\begin{aligned}
& =\frac{1}{2} R^{2}-\frac{\lambda}{2 \lambda_{k}} R^{2}+\frac{1}{2} \epsilon^{2} R^{2} \\
& =\frac{1}{2} R^{2}\left(1-\frac{\lambda}{\lambda_{k}}+\epsilon^{2}\right)
\end{aligned}
$$

como escolhemos $\epsilon^{2}<\frac{\lambda}{\lambda_{k}}-1$, temos que $I(v)<0$.

- Por fim, se $v=w+\epsilon R \widetilde{e} \in \Gamma_{3}$,

$$
\begin{aligned}
I(v)= & I(w+\epsilon R \widetilde{e}) \\
= & I(w+R e) \\
= & \frac{1}{2}\|w\|_{E}^{2}-\frac{\lambda}{2}\|w\|_{2}^{2}+\frac{1}{2} R^{2}\|e\|_{E}^{2}-\frac{\lambda}{2} R^{2}\|e\|_{2}^{2} \\
& \quad-\frac{R^{p+1}}{p+1} \int_{\Omega}\left(\frac{w}{R}+e+\frac{u_{t}}{R}\right)_{+}^{p+1} d x \\
\leq & \frac{1}{2}\left(1-\frac{\lambda}{\lambda_{k}}\right)\|w\|_{E}^{2}+\frac{1}{2} \epsilon^{2} R^{2}-\frac{R^{p+1}}{p+1} \int_{\Omega}\left(\frac{w}{R}+e+\frac{u_{t}}{R}\right)_{+}^{p+1} d x \\
\leq & \frac{1}{2} \epsilon^{2} R^{2}-\frac{R^{p+1}}{p+1} \int_{\Omega}\left(\frac{w}{R}+e+\frac{u_{t}}{R}\right)_{+}^{p+1} d x .
\end{aligned}
$$

Note que $\frac{w}{R} \in W_{k}$ e que $\left\|\frac{w}{R}\right\|_{E} \leq 1$. Como $u_{t} \in C(\bar{\Omega})$ e é negativa, pelo lema 4.3 existem $R_{0}, \eta>0$ tais que para todo $R \geq R_{0}$ vale

$$
\int_{\Omega}\left(\frac{w}{R}+e+\frac{u_{t}}{R}\right)_{+}^{p+1} d x \geq \eta
$$

assim, temos que

$$
I(v) \leq \frac{1}{2} R^{2} \epsilon^{2}-\frac{\eta}{p+1} R^{p+1}
$$

Como $\epsilon>0$ está fixo e $p \in\left(1,2^{*}-1\right)$, podemos escolher $R>\max \left\{R_{0}, \frac{\rho}{\epsilon}\right\}$ suficientemente grande de modo a termos $I(v) \leq 0$.

Observe que os conjuntos $S_{\rho}$ e $Q_{R, \epsilon}$, obtidos, respectivamente, nos Lemas 4.4 e 4.5 são tais como os conjuntos em (2.1) no exemplo 2.4; logo, $S_{\rho}$ e $Q_{R, \epsilon}$ enlaçam.

Dos Lemas 4.4 e 4.5 segue que o funcional $I$ satisfaz as hipóteses do TEOREMA DE 
ENLACE. Logo podemos dizer que em um nível $\bar{c} \geq \alpha$ existe uma sequência $(P S)$ para $I$, onde $\alpha>0$ vem do Lema 4.4. Além disso, $\bar{c}$ pode ser caracterizado como

$$
\bar{c}=\inf _{h \in \Gamma} \max _{u \in Q_{R, \epsilon}} I(h(u))
$$

onde $\Gamma=\left\{h \in C(E, E):\left.h\right|_{\partial Q_{R, \epsilon}=I d}\right\}$; lembrando que $R, \epsilon>0$ são escolhidos de acordo com o Lema 4.5 .

\subsection{A condição $(P S)$}

Se denotarmos por $\left\{v_{n}\right\}_{n \in \mathbb{N}}$ a sequência $(P S)$ para $I$ no nível $\bar{c}$ obtida na seção 4.2 por meio do TEOREMA DE ENLACE, temos que $\left\{v_{n}\right\}_{n \in \mathbb{N}}$ satisfaz

$$
I\left(v_{n}\right) \underset{n \rightarrow \infty}{\longrightarrow} \bar{c} \quad \text { e } \quad I^{\prime}\left(v_{n}\right) \underset{n \rightarrow \infty}{\longrightarrow} 0 .
$$

Se pudermos mostrar que existem $v_{0} \in E$ e uma subsequência de $\left\{v_{n}\right\}_{n \in \mathbb{N}}$, que continuaremos denotando por $v_{n}$, tais que $v_{n} \underset{n \rightarrow \infty}{\longrightarrow} v_{0}$ em $E$, poderemos, então, dizer que $I\left(v_{0}\right)=\bar{c}$ e $I^{\prime}\left(v_{0}\right)=0$; isso, por sua vez, significaria que $v_{0}$ é um ponto crítico não nulo do funcional $I$, o que equivaleria a dizer que $v_{0}$ é uma solução fraca não trivial do problema $\left(P_{t}^{\prime}\right)$.

Nesta seção mostraremos que o funcional $I$ dado por (4.1) satisfaz a condição $(P S)$.

\section{Lema 4.6.}

$O$ funcional $I: E \rightarrow \mathbb{R}$ definido em (4.1) satisfaz a condição (PS).

\section{Prova.}

Dado $c \in \mathbb{R}$, seja $\left\{v_{n}\right\}_{n \in \mathbb{N}}$ uma sequência em $E$ satisfazendo

$$
I\left(v_{n}\right) \underset{n \rightarrow \infty}{\longrightarrow} c \quad \text { e } \quad I^{\prime}\left(v_{n}\right) \underset{n \rightarrow \infty}{\longrightarrow} 0 .
$$

Primeiro, mostraremos que $\left(v_{n}\right)$ é uma sequência limitada em $E$; para isso, suporemos que $\left\|v_{n}\right\|_{E} \underset{n \rightarrow \infty}{\longrightarrow} \infty$ e buscaremos chegar numa contradição.

De (4.2), para cada $n \in \mathbb{N}$, temos

$$
\begin{aligned}
I^{\prime}\left(v_{n}\right) \cdot v_{n} & =\int_{\Omega}\left|\nabla v_{n}\right|^{2} d x-\lambda \int_{\Omega} v_{n}^{2} d x-\int_{\Omega}\left(v_{n}+u_{t}\right)_{+}^{p} v_{n} d x \\
& =\int_{\Omega}\left|\nabla v_{n}\right|^{2} d x-\lambda \int_{\Omega} v_{n}^{2} d x-\int_{\Omega}\left(v_{n}+u_{t}\right)_{+}^{p+1} d x+\int_{\Omega}\left(v_{n}+u_{t}\right)_{+}^{p} u_{t} d x .
\end{aligned}
$$


Disso e de (4.1) obtemos

$$
I\left(v_{n}\right)-\frac{1}{2} I^{\prime}\left(v_{n}\right) \cdot v_{n}=\left(\frac{1}{2}-\frac{1}{p+1}\right) \int_{\Omega}\left(v_{n}+u_{t}\right)_{+}^{p+1} d x+\frac{1}{2} \int_{\Omega}\left(v_{n}+u_{t}\right)_{+}^{p}\left(-u_{t}\right) d x .
$$

Observe que no lado direito da igualdade acima temos uma soma de termos positivos.

Seja $M>0$ tal que $\left|I\left(v_{n}\right)\right| \leq M$. Para $n$ suficientemente grande, temos

$$
\left|I\left(v_{n}\right)-\frac{1}{2} I^{\prime}\left(v_{n}\right) \cdot v_{n}\right| \leq M+\frac{1}{2}\left\|I^{\prime}\left(v_{n}\right)\right\|_{E^{\prime}}\left\|v_{n}\right\|_{E} \leq M+\left\|v_{n}\right\|_{E}
$$

assim

$$
\left(\frac{1}{2}-\frac{1}{p+1}\right) \int_{\Omega}\left(v_{n}+u_{t}\right)_{+}^{p+1} d x \leq\left|I\left(v_{n}\right)-\frac{1}{2} I^{\prime}\left(v_{n}\right) \cdot v_{n}\right| \leq M+\left\|v_{n}\right\|_{E} .
$$

Seja $\frac{1}{C}=\left(\frac{1}{2}-\frac{1}{p+1}\right)$ e multipliquemos a desigualdade acima por $\frac{C}{\left\|v_{n}\right\|_{E}^{\frac{p+1}{p}}}$ para obtermos

$$
\int_{\Omega}\left(\frac{\left(v_{n}+u_{t}\right)_{+}^{p}}{\left\|v_{n}\right\|_{E}}\right)^{\frac{p+1}{p}} d x \leq \frac{C M}{\left\|v_{n}\right\|_{E}^{\frac{p+1}{p}}}+\frac{C}{\left\|v_{n}\right\|_{E}^{\frac{1}{p}}}
$$

Como estamos assumindo que $\left\|v_{n}\right\|_{E} \underset{n \rightarrow \infty}{\longrightarrow} \infty$, temos que $\frac{\left(v_{n}+u_{t}\right)_{+}^{p}}{\left\|v_{n}\right\|_{E}} \underset{n \rightarrow \infty}{\longrightarrow} 0$ em $L^{\frac{p+1}{p}}(\Omega)$.

Dado $\varphi \in E$, pelo item (i) do TEOREMA DE MERGULHO DE SOBOLEV, temos que $\varphi \in L^{p+1}(\Omega)$; sendo assim, usando a desigualdade de Hölder, obtemos

$$
\begin{aligned}
\int_{\Omega}\left|\left(\frac{\left(v_{n}+u_{t}\right)_{+}^{p}}{\left\|v_{n}\right\|_{E}}\right) \varphi\right| d x & \leq\left[\int_{\Omega}\left(\frac{\left(v_{n}+u_{t}\right)_{+}^{p}}{\left\|v_{n}\right\|_{E}}\right)^{\frac{p+1}{p}} d x\right]^{\frac{p}{p+1}}\|\varphi\|_{p+1} \\
& \leq\left(\frac{C M}{\left\|v_{n}\right\|_{E}^{\frac{p+1}{p}}}+\frac{C}{\left\|v_{n}\right\|_{E}^{\frac{p}{p}}}\right)^{\frac{p}{p+1}}\|\varphi\|_{p+1} \underset{n \rightarrow \infty}{\longrightarrow} 0
\end{aligned}
$$

e isso nos mostra que $\frac{\left(v_{n}+u_{t}\right)_{+}^{p}}{\left\|v_{n}\right\|_{E}} \underset{n \rightarrow \infty}{\longrightarrow} 0$ em $E^{\prime}=H^{-1}$.

Agora observe que para cada $\varphi \in E$, temos

$$
\left|\int_{\Omega} \nabla v_{n} \cdot \nabla \varphi d x-\lambda \int_{\Omega} v_{n} \varphi d x-\int_{\Omega}\left(v_{n}+u_{t}\right)_{+}^{p} \varphi d x\right|=\left|I^{\prime}\left(v_{n}\right) \cdot \varphi\right| \leq\left\|I^{\prime}\left(v_{n}\right)\right\| E_{E^{\prime}}|| \varphi \|_{E}
$$


dividindo tudo por $\left\|v_{n}\right\|_{E}$ e considerando $w_{n}=\frac{v_{n}}{\left\|v_{n}\right\|_{E}}$, obtemos

$$
\left|\int_{\Omega} \nabla w_{n} \cdot \nabla \varphi d x-\lambda \int_{\Omega} w_{n} \varphi d x-\int_{\Omega} \frac{\left(v_{n}+u_{t}\right)_{+}^{p}}{\left\|v_{n}\right\|_{E}} \varphi d x\right| \leq \frac{\left\|I^{\prime}\left(v_{n}\right)\right\|_{E^{\prime}}}{\left\|v_{n}\right\|_{E}}\|\varphi\|_{E} .
$$

Como $\left(w_{n}\right)$ é limitada, existe $w_{0} \in E$ tal que, passando para uma subsequência se necessário, temos

$$
\begin{aligned}
& w_{n} \rightarrow_{n \rightarrow \infty} w_{0} \quad \text { em } E, \\
& w_{n} \underset{n \rightarrow \infty}{\longrightarrow} w_{0} \quad \text { em } L^{q} \operatorname{com} q \in\left[1,2^{*}\right), \\
& w_{n} \underset{n \rightarrow \infty}{\longrightarrow} w_{0} \quad \text { q.t.p. em } \Omega,
\end{aligned}
$$

$\operatorname{logo}$, fazendo $n \rightarrow \infty$ em (4.8) e usando (4.6), obtemos

$$
\int_{\Omega} \nabla w_{0} \cdot \nabla \varphi d x-\lambda \int_{\Omega} w_{0} \varphi d x=0 \quad \forall \varphi \in E
$$

Podemos, então, concluir que $w_{0}$ é uma solução fraca do problema

$$
\begin{cases}-\Delta w=\lambda w, & \text { em } \Omega \\ w=0, & \text { sobre } \partial \Omega .\end{cases}
$$

Como $\lambda \notin \sigma(-\Delta)$, devemos ter $w_{0}=0$.

Considerando, agora, $\varphi=w_{n}$ em (4.8), temos

$$
\int_{\Omega}\left|\nabla w_{n}\right|^{2} d x-\lambda \int_{\Omega} w_{n}^{2} d x-\int_{\Omega} \frac{\left(v_{n}+u_{t}\right)_{+}^{p}}{\left\|v_{n}\right\|_{E}} w_{n} d x \leq \frac{\left\|I^{\prime}\left(v_{n}\right)\right\|_{E^{\prime}}}{\left\|v_{n}\right\|_{E}}
$$

e segue, usando ainda (4.6), que

$$
\begin{aligned}
0 & =\lim _{n \rightarrow \infty} \frac{\left\|I^{\prime}\left(v_{n}\right)\right\|_{E^{\prime}}}{\left\|v_{n}\right\|_{E}} \\
& \geq \lim _{n \rightarrow \infty}\left(\int_{\Omega}\left|\nabla w_{n}\right|^{2} d x-\lambda \int_{\Omega} w_{n}^{2} d x-\int_{\Omega} \frac{\left(v_{n}+u_{t}\right)_{+}^{p}}{\left\|v_{n}\right\|_{E}} w_{n} d x\right) \\
& =\lim _{n \rightarrow \infty}\left\|w_{n}\right\|_{E}^{2} \\
& =1,
\end{aligned}
$$


mas isso é um absurdo. Logo, a sequência $\left(v_{n}\right)$ é limitada.

Finalmente mostraremos que $\left(v_{n}\right)$ admite uma subsequência convergente em $E$. Como $\left(v_{n}\right)$ é limitada, existe $v_{0} \in E$ tal que, passando para uma subsequência se necessário, temos

$$
\begin{array}{ll}
v_{n} \rightarrow_{n \rightarrow \infty} v_{0} & \text { em } E \\
v_{n} \underset{n \rightarrow \infty}{\longrightarrow} v_{0} & \text { em } L^{q} \operatorname{com} q \in\left[1,2^{*}\right) \\
v_{n} \underset{n \rightarrow \infty}{\longrightarrow} v_{0} & \text { q.t.p. em } \Omega .
\end{array}
$$

Como $I^{\prime}\left(v_{n}\right) \underset{n \rightarrow \infty}{\longrightarrow} 0$ em $E^{\prime}$ e $\left(v_{n}\right)$ é limitada em $E$ temos que

$$
\begin{aligned}
I^{\prime}\left(v_{n}\right) \cdot\left(v_{0}-v_{n}\right)= & \int_{\Omega} \nabla v_{n} \cdot \nabla\left(v_{0}-v_{n}\right) d x-\lambda \int_{\Omega} v_{n}\left(v_{0}-v_{n}\right) d x \\
& -\int_{\Omega}\left(v_{n}+u_{t}\right)_{+}^{p}\left(v_{0}-v_{n}\right) d x \rightarrow 0 .
\end{aligned}
$$

Lembrando que $E \hookrightarrow L^{p+1}(\Omega)$, usamos a desigualdade de Hölder com os expoentes conjugados $\frac{p+1}{p}$ e $p+1$ para obtermos

$$
\left|\int_{\Omega}\left(v_{n}+u_{t}\right)_{+}^{p}\left(v_{n}-v_{0}\right) d x\right| \leq\left\|\left(v_{n}+u_{t}\right)_{+}||_{p+1}^{p}\right\| v_{n}-v_{0} \|_{p+1} ;
$$

de (4.10) segue que

$$
\left|\int_{\Omega}\left(v_{n}+u_{t}\right)_{+}^{p}\left(v_{n}-v_{0}\right) d x\right| \underset{n \rightarrow \infty}{\longrightarrow} 0
$$

Usando ainda (4.10), com $q=2$, de (4.12) obtemos

$$
\lim _{n \rightarrow \infty} \int_{\Omega} \nabla v_{n} \cdot \nabla v_{0} d x=\lim _{n \rightarrow \infty} \int_{\Omega}\left|\nabla v_{n}\right|^{2} d x .
$$

De (4.9) segue que

$$
\begin{aligned}
\lim _{n \rightarrow \infty} \int_{\Omega} \nabla v_{n} \cdot \nabla v_{0} d x & =\lim _{n \rightarrow \infty}\left\langle v_{n}, v_{0}\right\rangle_{E} \\
& =\left\langle v_{0}, v_{0}\right\rangle_{E} \\
& =\int_{\Omega}\left|\nabla v_{0}\right|^{2} d x
\end{aligned}
$$


ou seja, $\left\|v_{n}\right\|_{E} \rightarrow\left\|v_{0}\right\|_{E}$, que, junto com (4.9), implica $v_{n} \underset{n \rightarrow \infty}{\longrightarrow} v_{0}$ em $E$.

\subsection{Prova do Teorema 4.1}

Na seção 4.2 mostramos que o funcional $I$ dado por 4.1 possui a estrutura de enlace, o que nos forneceu uma sequência $(P S)$ para $I$ no nível $\bar{c}>0$ que denotaremos por $\left\{v_{n}\right\}_{n \in \mathbb{N}}$. Tal sequência satisfaz

$$
I\left(v_{n}\right) \underset{n \rightarrow \infty}{\longrightarrow} \bar{c} \quad \text { e } \quad I^{\prime}\left(v_{n}\right) \underset{n \rightarrow \infty}{\longrightarrow} 0 .
$$

Como na seção 4.3 mostramos que $I$ satisfaz a condição $(P S)$, podemos concluir, como queríamos, que $I$ possui um ponto crítico $v_{0} \in E \backslash\{0\}$. Tal ponto crítico é solução fraca não trivial do problema $\left(P_{t}^{\prime}\right)$; portanto, temos que a função $v_{0}+u_{t}$ é a segunda solução fraca do problema $P^{\prime}$. Assim fica provado o Teorema 4.1. 


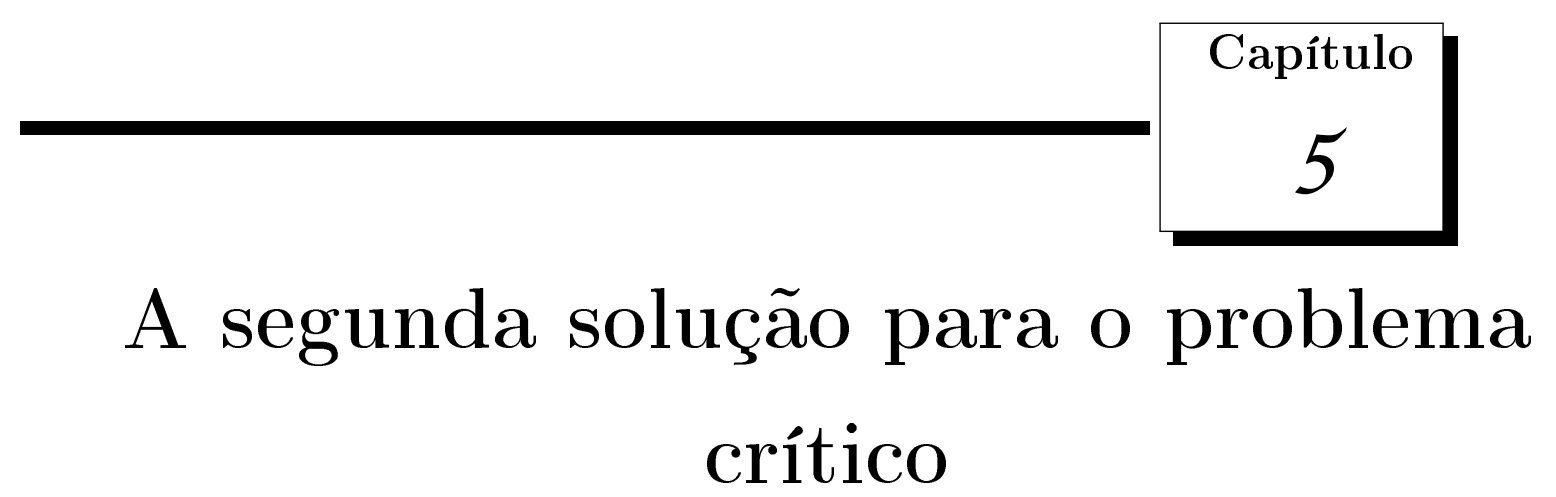

\subsection{Apresentação do problema}

Estudamos nesta seção o problema elíptico crítico abordado em [CR02].

Seja $\Omega \subset \mathbb{R}^{N}(N \geq 3)$ um domínio limitado com fronteira suave. Consideraremos o problema elíptico superlinear com condição de fronteira de Dirichlet

$$
\left\{\begin{array}{lc}
-\Delta u=\lambda u+u_{+}^{2^{*}-1}+g\left(x, u_{+}\right)+f(x), & \text { em } \Omega \\
u=0, & \text { sobre } \partial \Omega .
\end{array}\right.
$$

onde $\lambda>\lambda_{1}$ é tal que $\lambda \notin \sigma(-\Delta) ; f=h+t \phi_{1}$, com $h \in L^{r}(\Omega)$ e $r>N$, e $g: \bar{\Omega} \times \mathbb{R} \rightarrow \mathbb{R}_{+}$ é uma função que satisfaz as seguintes condições:

$\left(g_{1}\right) g$ é contínua;

$\left(g_{2}\right) g(x, s) \equiv 0$ para todo $s \leq 0$

$\left(g_{3}\right) g$ tem crescimento subcrítico no infinito, ou seja, existem $C_{g}>0$ e $p+1 \in\left(2,2^{*}\right)$ tais que

$$
0 \leq g(x, s) \leq C_{g}|s|^{p} \quad \forall s \in \mathbb{R}
$$


$\left(g_{4}\right)$ Para $N=3,4,5$ devemos assumir em adição que existem $D_{g}>0$ e $q \in \mathbb{R}$ com

$$
2^{*}>q+1> \begin{cases}\frac{2}{3} 2^{*}, & \text { se } N=4,5 \\ \frac{9}{2}, & \text { se } N=3\end{cases}
$$

tais que

$$
g\left(x, s_{+}\right) \geq D_{g} s_{+}^{q} \quad \forall s \in \mathbb{R} .
$$

As condições $\left(g_{2}\right)$ e $\left(g_{3}\right)$ implicam que a função real de variável real $k(s)=\lambda s+s_{+}^{2^{*}-1}+$ $g\left(x, s_{+}\right)$interage com todos os autovalores do laplaciano exceto os primeiros no seguinte sentido

$$
\lambda_{1}<\lambda=\lim _{s \rightarrow-\infty} \frac{k(s)}{s}<\lim _{s \rightarrow \infty} \frac{k(s)}{s}=\infty .
$$

Tal fato, de acordo com a literatura, enquadra o problema $(P)$ no conjunto de problemas chamados de tipo Ambrosetti-Prodi, ver, por exemplo, [DFJ99].

Objetivamos provar os seguintes teoremas:

\section{Teorema 5.1.}

Seja $N \geq 6$. Dado $h \in L^{r}(\Omega)$, se g satisfaz as condições $\left(g_{1}\right)-\left(g_{3}\right)$, então existe $T=T(h)$ tal que para todo $t>T$ o problema $(P)$ tem pelo menos duas soluções.

\section{Teorema 5.2.}

Seja $3 \leq N \leq 5$. Dado $h \in L^{r}(\Omega)$, se g satisfaz as condições ( $\left.g_{1}\right)$ - $\left(g_{4}\right)$, então existe $T=T(h)$ tal que para todo $t>T$ o problema $(P)$ tem pelo menos duas soluções.

A primeira solução é a solução negativa $u_{t}$ obtida do Teorema 3.1, onde $t>T(h)$ é dado.

Se procedermos de modo análogo ao feito no início do capítulo 4, devemos procurar uma segunda solução de $(P)$ na forma $u=v+u_{t}$ observando que $u$ será uma solução fraca de $(P)$ se, e só se, $v$ for solução fraca do problema

$$
\begin{cases}-\Delta v=\lambda v+\left(v+u_{t}\right)_{+}^{2^{*}-1}+g\left(x,\left(v+u_{t}\right)_{+}\right), & \text {em } \Omega \\ v=0, & \text { sobre } \partial \Omega .\end{cases}
$$

No entanto, no caso crítico precisamos enfrentar uma dificuldade a mais que é a falta de compacidade do mergulho de Sobolev

$$
E \hookrightarrow L^{2^{*}}(\Omega)
$$


Isso significa que o funcional associado à equação (5.1) pode não satisfazer a condição $(P S)_{c}$, ou seja, pode existir um nível minimax $c$ sem que exista um ponto crítico correspondente.

Desde o trabalho de Brézis e Nirenberg [BN83], é conhecido o fato de ser possível contornar a fralta de compacidade em certos níveis $c$; desta maneira, se torna importante estimar com precisão o nível minimax do funcional para poder mostrar a existência de um ponto crítico correspondente. Para estimar este nível é, em geral, necessário explorar as propriedades das funções extremais $\Phi_{\epsilon}$ do mergulho de Sobolev, que serão apresentadas mais adiante. Inspirados nessas ideias, de Figueiredo e Yang provaram a existência de uma segunda solução fraca para o problema $(P)$ para o caso $N \geq 7, \operatorname{com} g \equiv 0$, em [DFJ99]. No artigo citado, além de ser verificado que o funcional associado a $(P)$ satisfaz as hipóteses do TEOREMA DE ENLACE, verificou-se que existem níveis minimax $c$ no intervalo $\left(0, \frac{1}{N} S^{\frac{N}{2}}\right)$, onde $S$, que será definida mais à frente, é a melhor constante que realiza o mergulho de Sobolev $E \hookrightarrow L^{2^{*}}(\Omega)$. Do TEOREMA DE ENLACE segue a existência de uma sequência $(P S)$ para $I$ no nível $c$. O fato de $c \in\left(0, \frac{1}{N} S^{\frac{N}{2}}\right)$ é útilizado tanto para mostrar que a sequência converge a uma solução fraca do problema, quanto para garantir que tal solução não é a trivial.

No intuito de melhorar os resultados obtidos em [DFJ99], será feita aqui uma abordagem diferente. A idéia consiste em "cavar buracos" na solução negativa $u_{t}$ e nas autofunções do Laplaciano, e de concentrar as funções extremais $\Phi_{\epsilon}$ em bolas pequenas, de modo a obtermos funções com propriedades similares às das originais, mas com suportes disjuntos. Essas manipulações criam alguns erros, mas eles são mais fáceis de estimar que os termos mistos que surgem em expressões como $\left(v+u_{t}+\Phi_{\epsilon}\right)_{+}^{2^{*}}$ quando seus suportes não são disjuntos. Mover esses "pequenos buracos" para próximo da fronteira, onde a função $\left|u_{t}\right|$ é pequena, nos possibilitará obter estimativas melhores que as encontradas em [DFJ99]. É interessante observar que com essa abordagem já é possível estender o resultado em [DFJ99] para o caso $N=6$; de fato, observe que as hipóteses $\left(g_{1}\right)$ - $\left(g_{3}\right)$ são satisfeitas pela função $g \equiv 0$. Para os casos $N=3,4,5$, será necessário assumir a hipótese $\left(g_{4}\right)$, pois a função $g$ será indispensável para obter o resultado desejado nestas dimensões.

\subsection{Reformulação do problema}

Nesta seção será descrito o modo como reformularemos o problema $(P)$ a fim de encontrar uma segunda solução.

Dado $m \in \mathbb{N}$ grande escolheremos $x_{m} \in \Omega$ tal que $d\left(x_{m}, \partial \Omega\right)=\frac{4}{m}$ e consideraremos 
$\eta_{m} \in C^{\infty}(\bar{\Omega})$ tal que

$$
\begin{aligned}
& 0 \leq \eta_{m} \leq 1 \\
& \eta_{m}(x)= \begin{cases}0, & \text { se } x \in B_{\frac{1}{m}}\left(x_{m}\right) \\
1, & \text { se } x \in \bar{\Omega} \backslash B_{\frac{2}{m}}\left(x_{m}\right),\end{cases} \\
& \left\|\nabla \eta_{m}\right\|_{\infty} \leq 2 m
\end{aligned}
$$

Definimos, então, as funções $u_{t}^{m}(x)=\eta_{m}(x) u_{t}(x)$.

Como $u_{t} \in W^{2,2}(\Omega), 1-\eta_{m} \in C_{0}^{\infty}(\Omega)$ e observando que $u_{t}^{m}=u_{t}-\left(1-\eta_{m}\right) u_{t}$, pela Proposição 11.16 de [Bie10] temos que $u_{t}^{m} \in W^{2,2}(\Omega)$; em particular, $u_{t}^{m} \in W^{1,2}(\Omega)$. Além disso, como $u_{t} \in C(\bar{\Omega})$, temos que $u_{t}^{m} \in C(\bar{\Omega})$; usando agora o Teorema 11.24 de [Bie10], concluímos que $u_{t}^{m} \in E$.

Assim, em vez de procurarmos diretamente soluções de $(P)$ como em [DFJ99], procuraremos soluções de $(P)$ na forma $u=v+u_{t}^{m}$, onde $v$ satisfaz

$$
\begin{cases}-\Delta v-\Delta u_{t}^{m}=\lambda v+\lambda u_{t}^{m}+\left(v+u_{t}^{m}\right)_{+}^{2^{*}-1}+g\left(x,\left(v+u_{t}^{m}\right)_{+}\right)+f, & \text { em } \Omega \\ v=0, & \text { sobre } \partial \Omega ;\end{cases}
$$

de fato, $u$ é uma solução fraca de $(P)$ se, e só se, $v$ for solução fraca do problema acima.

Definindo $f_{m}=-\Delta u_{t}^{m}-\lambda u_{t}^{m} \in L^{2}(\Omega),(5.2)$ torna-se equivalente a

$$
\begin{cases}-\Delta v=\lambda v+\left(v+u_{t}^{m}\right)_{+}^{2^{*}-1}+g\left(x,\left(v+u_{t}^{m}\right)_{+}\right)+f-f_{m}, & \text { em } \Omega \\ v=0, & \text { sobre } \partial \Omega\end{cases}
$$

Temos então que $v \equiv u_{t}-u_{t}^{m}$ é uma solução fraca trivial de $\left(P_{m}\right)$, no sentido de que ela corresponde à solução negativa $u_{t}$ que já temos. Para encontrarmos uma segunda solução fraca de $\left(P_{m}\right)$, devemos procurar pontos críticos $v \not \equiv u_{t}-u_{t}^{m}$ do funcional $I_{m} \in C^{1}(E, \mathbb{R})$ dado por

$$
\begin{aligned}
I_{m}(v)= & \frac{1}{2} \int_{\Omega}\left(|\nabla v|^{2}-\lambda v^{2}\right) d x-\frac{1}{2^{*}} \int_{\Omega}\left(v+u_{t}^{m}\right)_{+}^{2^{*}} d x \\
& -\int_{\Omega} G\left(x,\left(v+u_{t}^{m}\right)_{+}\right) d x-\left(F-F_{m}\right)(v),
\end{aligned}
$$


onde $G: \bar{\Omega} \times \mathbb{R} \rightarrow \mathbb{R}_{+}$é a primitiva de $g$, ou seja,

$$
G(x, t)=\int_{0}^{t} g(x, s) d s
$$

enquanto $F, F_{m}: E \rightarrow \mathbb{R}$ são dadas por

$$
\begin{gathered}
F(v)=\int_{\Omega} f v d x=\int_{\Omega}\left(\nabla u_{t} \nabla v-\lambda u_{t} v\right) d x, \\
F_{m}(v)=\int_{\Omega} f_{m} v d x=\int_{\Omega}\left(\nabla u_{t}^{m} \nabla v-\lambda u_{t}^{m} v\right) d x .
\end{gathered}
$$

A derivada do funcional $I$ é dada por

$$
\begin{aligned}
I_{m}^{\prime}(v) \cdot \varphi= & \int_{\Omega} \nabla v \nabla \varphi d x-\lambda \int_{\Omega} v \varphi d x-\int_{\Omega}\left(v+u_{t}^{m}\right)_{+}^{2^{*}-1} \varphi d x \\
& -\int_{\Omega} g\left(x,\left(v+u_{t}^{m}\right)_{+}\right) \varphi d x-\left(F-F_{m}\right)(\varphi) .
\end{aligned}
$$

\subsection{Estimando a pertubação $f-f_{m}$}

Nesta seção mostraremos como estimar a perturbação dada pelo termo $f-f_{m}$.

\section{Lema 5.3.}

Se $N \geq 3$ e $m \in \mathbb{N}$ é suficientemente grande, então

$$
\begin{aligned}
& \left\|u_{t}-u_{t}^{m}\right\|_{E} \leq C_{1}(N) m^{-\frac{N}{2}} \\
& \left|\left(F-F_{m}\right)(\varphi)\right| \leq C_{2}(N) m^{-\frac{N}{2}}\|\varphi\|_{E}, \quad \forall \varphi \in E
\end{aligned}
$$

\section{Prova.}

Como $\Omega$ é de classe $C^{\infty}$ e $x_{m} \in \Omega$ foi escolhido de modo que $d\left(x_{m}, \partial \Omega\right)=\inf _{y \in \partial \Omega}\left|y-x_{m}\right|=\frac{4}{m}$, então $B_{\frac{4}{m}}\left(x_{m}\right) \subset \Omega$. No capítulo 3 vimos que $u_{t} \in C^{1,1-\frac{N}{r}}(\bar{\Omega})$. Como $\Omega$ é limitado, temos que $c_{t}=\left\|\nabla u_{t}\right\|_{\infty}<\infty$; assim dados $x \in B_{\frac{4}{m}}\left(x_{m}\right)$ e $y \in \partial \Omega$, pela desigualdade do valor médio, obtemos

$$
\left|u_{t}(x)\right| \leq c_{t}|x-y| \leq c_{t}\left[\left|x-x_{m}\right|+\left|y-x_{m}\right|\right] \leq c_{t}\left[\frac{4}{m}+\left|y-x_{m}\right|\right] .
$$

Observe que na desigualdade resultante um lado depende apenas de $x$ e o outro depende 
apenas de $y$, sendo que $\left|y-x_{m}\right| \geq 4 / m$, logo

$$
\left|u_{t}(x)\right| \leq \inf _{y \in \partial \Omega}\left\{c_{t}\left[\frac{4}{m}+\left|y-x_{m}\right|\right]\right\}=\frac{8 c_{t}}{m} \quad \forall x \in B_{\frac{4}{m}}\left(x_{m}\right)
$$

Assim, podemos fazer a seguinte estimativa, onde para aliviar a notação omitimos de especificar o centro $x_{m}$ das bolas:

$$
\begin{aligned}
|| u_{t}-u_{t}^{m} \|_{E}^{2}= & \int_{\Omega}\left|\nabla\left(u_{t}-u_{t}^{m}\right)\right|^{2} d x \\
= & \int_{\Omega}\left|\left(1-\eta_{m}\right) \nabla u_{t}-u_{t} \nabla \eta_{m}\right|^{2} d x \\
= & \int_{\Omega}\left|1-\eta_{m}\right|^{2}\left|\nabla u_{t}\right|^{2} d x-2 \int_{\Omega}\left(1-\eta_{m}\right) u_{t} \nabla u_{t} \cdot \nabla \eta_{m} d x \\
& +\int_{\Omega}\left|u_{t}\right|^{2}\left|\nabla \eta_{m}\right|^{2} d x \\
\leq & \int_{B_{\frac{2}{m}}}\left|1-\eta_{m}\right|^{2}\left|\nabla u_{t}\right|^{2} d x+2 \int_{B_{\frac{2}{m}} \backslash B_{\frac{1}{m}}}\left|1-\eta_{m}\right|\left|u_{t}\right|\left|\nabla u_{t}\right|\left|\nabla \eta_{m}\right| d x \\
& +\int_{B_{\frac{2}{m}} \backslash B_{\frac{1}{m}}}\left|u_{t}\right|^{2}\left|\nabla \eta_{m}\right|^{2} d x .
\end{aligned}
$$

Como nas regiões de integração acima vale (5.6) e lembrando que $\left\|\nabla \eta_{m}\right\|_{\infty} \leq 2 m$, podemos estimar

$$
\begin{aligned}
\left\|u_{t}-u_{t}^{m}\right\|_{E}^{2} & \leq c_{t}^{2} \frac{\omega_{N}}{N} \frac{2^{N}}{m^{N}}+2 \frac{8 c_{t}}{m} c_{t} 2 m \frac{\omega_{N}}{N} \frac{2^{N}-1}{m^{N}}+\frac{64 c_{t}^{2}}{m^{2}} 4 m^{2} \frac{\omega_{N}}{N} \frac{2^{N}-1}{m^{N}} \\
& =\left[2^{N}+32\left(2^{N}-1\right)+256\left(2^{N}-1\right)\right] c_{t}^{2} \frac{\omega_{N}}{N} m^{-N} \\
& =C(N) m^{-N} .
\end{aligned}
$$

A segunda estimativa é agora uma simples consequência da anterior, de fato

$$
\begin{aligned}
\left|\left(F-F_{m}\right)(\varphi)\right| & =\left|\int_{\Omega} \nabla\left(u_{t}-u_{t}^{m}\right) \cdot \nabla \varphi d x-\lambda \int_{\Omega}\left(u_{t}-u_{t}^{m}\right) \varphi d x\right| \\
& \leq\left\|u_{t}-u_{t}^{m}\right\|_{E}\|\varphi\|_{E}+\lambda\left\|u_{t}-u_{t}^{m}\right\|_{2}\|\varphi\|_{2} \\
& =\left(1+\lambda C^{2}\right)\left\|u_{t}-u_{t}^{m}\right\|_{E}\|\varphi\|_{E} \\
& \leq\left(1+\lambda C^{2}\right) C_{1} m^{-\frac{N}{2}}\|\varphi\|_{E}
\end{aligned}
$$

para todo $\varphi \in E$. 


\subsection{Funções extremais para o mergulho de Sobolev}

Nesta seção iremos apresentar as funções extremais do mergulho de Sobolev, mencionadas na seção 5.1. Além disso, será demonstrado um lema com estimativas envolvendo tais funções, o qual será bastante útil ao longo da demonstração dos Teoremas 5.1 e 5.2.

Pelo item (i) do TEOREMA DE MERGULHO DE SOBOLEV temos que $E \hookrightarrow L^{2^{*}}(\Omega)$, ou seja, existe $C>0$ tal que $\|u\|_{2^{*}}^{2} \leq C\|u\|_{E}^{2}$. Definimos, então, a melhor constante de Sobolev como sendo $S=\inf _{u \in E \backslash\{0\}} \frac{\|u\|_{E}^{2}}{\|u\|_{2^{*}}^{2}}$.

\section{Observação 5.4.}

- $S$ é independente de $\Omega$ e depende somente de $N$, ver a observação 4.5 no capítulo 1 de [Str08].

- Quando $\Omega=\mathbb{R}^{N}$, o ínfimo é atingido pelas funções

$$
\Phi_{\epsilon}(x)=\left(\frac{\sqrt{N(N-2)} \epsilon}{\epsilon^{2}+|x|^{2}}\right)^{\frac{N-2}{2}} \quad \operatorname{com} \epsilon>0
$$

que verificam

$$
\left\|\Phi_{\epsilon}\right\|_{W_{0}^{1,2}\left(\mathbb{R}^{N}\right)}^{2}=S|| \Phi_{\epsilon} \|_{L^{2^{*}\left(\mathbb{R}^{N}\right)}}^{2} .
$$

As funções $\Phi_{\epsilon}$ são chamadas de funções extremais do mergulho de Sobolev. Verifica-se que essas funções satisfazem a equação

$$
\begin{cases}-\Delta u=|u|^{2^{*}-1}, & \text { em } \mathbb{R}^{N} \\ u \rightarrow 0, & \operatorname{com}|x| \rightarrow \infty\end{cases}
$$

Da formulação fraca de (5.8), segue que $\left\|\Phi_{\epsilon}\right\|_{W_{0}^{1,2}\left(\mathbb{R}^{N}\right)}^{2}=\left\|\Phi_{\epsilon}\right\|_{L^{2^{*}\left(\mathbb{R}^{N}\right)}}^{2^{*}}$; combinando essa igualdade com (5.7), obtemos

$$
S\left\|\Phi_{\epsilon}\right\|_{L^{2^{*}\left(\mathbb{R}^{N}\right)}}^{2-2^{*}}=S \frac{\left\|\Phi_{\epsilon}\right\|_{L^{2^{*}}\left(\mathbb{R}^{N}\right)}^{2}}{\left\|\Phi_{\epsilon}\right\|_{L^{2^{*}}\left(\mathbb{R}^{N}\right)}^{2^{*}}}=\frac{\left\|\Phi_{\epsilon}\right\|_{W_{0}^{1,2}\left(\mathbb{R}^{N}\right)}^{2}}{\left\|\Phi_{\epsilon}\right\|_{L^{2^{*}}\left(\mathbb{R}^{N}\right)}^{2^{*}}}=\frac{\left\|\Phi_{\epsilon}\right\|_{L^{2^{*}\left(\mathbb{R}^{N}\right)}}^{2^{*}}}{\left\|\Phi_{\epsilon}\right\|_{L^{2^{*}\left(\mathbb{R}^{N}\right)}}^{2^{*}}}=1
$$


ou seja, $S=\left\|\Phi_{\epsilon}\right\|_{L^{2^{2}\left(\mathbb{R}^{N}\right)}}^{2^{*}-2}$, o que nos dá

$$
\left\|\Phi_{\epsilon}\right\|_{W_{0}^{1,2}\left(\mathbb{R}^{N}\right)}^{2}=\left\|\Phi_{\epsilon}\right\|_{L^{2^{*}}\left(\mathbb{R}^{N}\right)}^{2^{*}}=\left\|\Phi_{\epsilon}\right\|_{L^{2^{*}\left(\mathbb{R}^{N}\right)}}^{\frac{N}{2} \frac{4}{N-2}}=\left(\left\|\Phi_{\epsilon}\right\|_{L^{2^{*}}\left(\mathbb{R}^{N}\right)}^{2^{*}}\right)^{\frac{N}{2}}=S^{\frac{N}{2}}
$$

Como $\left.\Phi_{\epsilon}\right|_{\Omega} \notin E$, precisamos "cortar" essas funções de modo apropriado. Ao fazer isso aproveitaremos também para manter o suporte destas funções "cortadas" desjuntos dos das outras definidas anteriormente, o que facilitará bastante as estimativas que precisaremos fazer. Sejam então $x_{m} \in \Omega$ os pontos escolhidos na seção 5.2. Considere $\xi_{m} \in C_{0}^{\infty}\left(\mathbb{R}^{N}\right)$ tais que

$$
\begin{aligned}
& 0 \leq \xi_{m}(x) \leq 1 \\
& \xi_{m}(x)= \begin{cases}1, & \text { se } x \in B_{\frac{1}{2 m}}\left(x_{m}\right) \\
0, & \text { se } x \in \mathbb{R}^{N} \backslash B_{\frac{1}{m}}\left(x_{m}\right)\end{cases} \\
& \left\|\nabla \xi_{m}\right\|_{\infty} \leq 4 m
\end{aligned}
$$

Definimos, então, as funções $\Phi_{\epsilon, m}(x)=\xi_{m}(x) \Phi_{\epsilon}\left(x-x_{m}\right)$; desta forma, $\left.\Phi_{\epsilon, m}\right|_{\Omega} \in E$. Mesmo quando estivermos considerando a restrição de tais funções a $\Omega$, continuaremos denotando-as por $\Phi_{\epsilon, m}$. Obtemos, desse modo, as estimativas do lema a seguir cuja demonstração foi inspirada nos trabalhos [BN83] e [Gho93].

\section{Lema 5.5.}

Suponha que $m \rightarrow \infty, \epsilon \rightarrow 0$ e $\epsilon m \rightarrow 0$. Temos, então, que

(a) ||$\left|\Phi_{\epsilon, m} \|_{E}^{2}-S^{\frac{N}{2}}\right| \leq D_{1}(N)(\epsilon m)^{N-2}$

(b) $\left.||\left|\Phi_{\epsilon, m}\right|\right|_{2^{*}} ^{2^{*}}-S^{\frac{N}{2}} \mid \leq D_{2}(N)(\epsilon m)^{N}$

(c) $\left\|\Phi_{\epsilon, m}\right\|_{s}^{s} \geq D_{3}(N, s) \epsilon^{N-\frac{N-2}{2} s}$

(d)

$$
\left\|\Phi_{\epsilon, m}\right\|_{2}^{2} \leq \begin{cases}D(N) \epsilon^{2}, & \text { se } N \geq 5 \\ D(4) \epsilon^{2}|\log (\epsilon m)|, & \text { se } N=4 \\ D(3) \frac{\epsilon}{m}, & \text { se } N=3\end{cases}
$$




\section{Prova.}

Nesta demonstração assumiremos que o centro $x_{m}$ das bolas seja a origem, a fim de não sobrecarregar a notação.

- Prova dos item (a):

Primeiro observe que

$$
\nabla \Phi_{\epsilon}(x)=-\frac{(N-2)(\sqrt{N(N-2)} \epsilon)^{\frac{N-2}{2}}}{\left(\epsilon^{2}+|x|^{2}\right)^{\frac{N}{2}}} x
$$

De (5.9) temos

$$
\begin{aligned}
\mid\left\|\Phi_{\epsilon, m}\right\|_{E}^{2}-S^{\frac{N}{2} \mid}= & \left.\left|\int_{\Omega}\right| \nabla \Phi_{\epsilon, m}(x)\right|^{2} d x-S^{\frac{N}{2}} \mid \\
= & \left.\left|\int_{B_{\frac{1}{2 m}}}\right| \nabla \Phi_{\epsilon}(x)\right|^{2} d x+\int_{B_{\frac{1}{m}} \backslash B_{\frac{1}{2 m}}}\left|\xi_{m}(x) \nabla \Phi_{\epsilon}(x)+\Phi_{\epsilon}(x) \nabla \xi_{m}(x)\right|^{2} d x-S^{\frac{N}{2}} \mid \\
\leq & \left.\left|\int_{B_{\frac{1}{2 m}}}\right| \nabla \Phi_{\epsilon}(x)\right|^{2} d x-\int_{\mathbb{R}^{N}}\left|\nabla \Phi_{\epsilon}(x)\right|^{2} d x \mid \\
& +\left|\int_{B_{\frac{1}{m}} \backslash B_{\frac{1}{2 m}}}\right| \xi_{m}(x) \nabla \Phi_{\epsilon}(x)+\left.\Phi_{\epsilon}(x) \nabla \xi_{m}(x)\right|^{2} d x \mid \\
\leq & \int_{\mathbb{R}^{N} \backslash B_{\frac{1}{2 m}}}\left|\nabla \Phi_{\epsilon}(x)\right|^{2} d x+4 \int_{B_{\frac{1}{m}} \backslash B_{\frac{1}{2 m}}}\left|\xi_{m}(x) \nabla \Phi_{\epsilon}(x)\right|^{2} d x \\
& +4 \int_{B_{\frac{1}{m}} \backslash B_{\frac{1}{2 m}}}\left|\Phi_{\epsilon}(x) \nabla \xi_{m}(x)\right|^{2} d x .
\end{aligned}
$$

Da definição de $\Phi_{\epsilon}$, de (5.10) e do fato de $\left\|\nabla \xi_{m}\right\|_{\infty} \leq 4 m$, segue que

$$
\begin{aligned}
\left.||\left|\Phi_{\epsilon, m}\right|\right|_{E} ^{2}-S^{\frac{N}{2} \mid \leq} & \int_{\mathbb{R}^{N} \backslash B_{\frac{1}{2 m}}}(N-2)^{2} \frac{(\sqrt{N(N-2)} \epsilon)^{N-2}}{\left(\epsilon^{2}+|x|^{2}\right)^{N}}|x|^{2} d x \\
& +4 \int_{B_{\frac{1}{m}} \backslash B_{\frac{1}{2 m}}}(N-2)^{2} \frac{(\sqrt{N(N-2)} \epsilon)^{N-2}}{\left(\epsilon^{2}+|x|^{2}\right)^{N}}|x|^{2} d x \\
& +64 m^{2} \int_{B_{\frac{1}{m}} \backslash B_{\frac{1}{2 m}}}\left(\frac{\sqrt{N(N-2)} \epsilon}{\epsilon^{2}+|x|^{2}}\right)^{N-2} d x
\end{aligned}
$$




$$
\begin{aligned}
& \leq 5 \int_{\mathbb{R}^{N} \backslash B_{\frac{1}{2 m}}}(N-2)^{2} \frac{(\sqrt{N(N-2)} \epsilon)^{N-2}}{\left(\epsilon^{2}+|x|^{2}\right)^{N}}|x|^{2} d x \\
& \quad+64 m^{2} \int_{B_{\frac{1}{m} \backslash B_{\frac{1}{2 m}}}}\left(\frac{\sqrt{N(N-2)} \epsilon}{\epsilon^{2}+|x|^{2}}\right)^{N-2} d x .
\end{aligned}
$$

Como $|x|^{2} \leq \epsilon^{2}+|x|^{2}$

$$
\begin{aligned}
\mid\left\|\Phi_{\epsilon, m}\right\|_{E}^{2}-S^{\frac{N}{2} \mid \leq} & 5 \int_{\mathbb{R}^{N} \backslash B_{\frac{1}{2 m}}}(N-2)^{2} \frac{(\sqrt{N(N-2)} \epsilon)^{N-2}}{|x|^{2 N-2}} d x \\
& +64 m^{2} \int_{B_{\frac{1}{m} \backslash B_{\frac{1}{2 m}}}} \frac{(\sqrt{N(N-2)} \epsilon)^{N-2}}{|x|^{2 N-4}} d x \\
\leq & {[N(N-2)]^{\frac{N-2}{2}}\left[5(N-2)^{2} \int_{\mathbb{R}^{N} \backslash B_{\frac{1}{2 m}}} \frac{1}{|x|^{2 N-2}} d x\right.} \\
& \left.+64 m^{2} \int_{B_{\frac{1}{m} \backslash B_{\frac{1}{2 m}}}} \frac{1}{|x|^{2 N-4}} d x\right] \epsilon^{N-2} .
\end{aligned}
$$

Observe que

$$
\begin{aligned}
& \int_{\mathbb{R}^{N} \backslash B_{\frac{1}{2 m}}} \frac{1}{|x|^{2 N-2}} d x=\frac{\omega_{N}}{N-2}(2 m)^{N-2}, \\
& m^{2} \int_{B_{\frac{1}{m} \backslash B_{\frac{1}{2 m}}}} \frac{1}{|x|^{2 N-4}} d x= \begin{cases}\frac{\omega_{N}}{2} m, & \text { se } N=3 ; \\
\omega_{N}(\log 2) m^{2}, & \text { se } N=4 ; \\
\frac{\omega_{N}}{N-4}\left(2^{N-4}-1\right) m^{N-2}, & \text { se } N \geq 5,\end{cases}
\end{aligned}
$$

logo, temos que ||$\left|\Phi_{\epsilon, m} \|_{E}^{2}-S^{\frac{N}{2}}\right| \leq D_{1}(N)(\epsilon m)^{N-2}$, provando o item (a).

- Prova dos item (b): 
Usando (5.9) e procedendo de modo similar ao que foi feito acima temos

$$
\begin{aligned}
\left|\left\|\Phi_{\epsilon, m}\right\|\right|_{2^{*}}^{2^{*}}-S^{\frac{N}{2}} \mid & =\left.\left|\int_{\Omega}\right| \Phi_{\epsilon, m}(x)\right|^{2^{*}} d x-S^{\frac{N}{2}} \mid \\
& =\left.\left|\int_{B_{\frac{1}{2 m}}}\right| \Phi_{\epsilon}(x)\right|^{2^{*}} d x+\int_{B_{\frac{1}{m}} \backslash B_{\frac{1}{2 m}}}\left|\xi_{m}(x) \Phi_{\epsilon}(x)\right|^{2^{*}} d x-\int_{\mathbb{R}^{N}}\left|\Phi_{\epsilon}(x)\right|^{2^{*}} d x \mid \\
& \leq \int_{\mathbb{R}^{N} \backslash B_{\frac{1}{2 m}}}\left|\Phi_{\epsilon}(x)\right|^{2^{*}} d x+\int_{B_{\frac{1}{m}} \backslash B_{\frac{1}{2 m}}}\left|\xi_{m}(x) \Phi_{\epsilon}(x)\right|^{2^{*}} d x \\
& \leq 2 \int_{\mathbb{R}^{N} \backslash B_{\frac{1}{2 m}}}\left|\Phi_{\epsilon}(x)\right|^{2^{*}} d x \\
& =2 \int_{\mathbb{R}^{N} \backslash B_{\frac{1}{2 m}}}\left(\frac{\sqrt{N(N-2)} \epsilon}{\epsilon^{2}+|x|^{2}}\right)^{N} d x \\
& \leq 2 \int_{\mathbb{R}^{N} \backslash B_{\frac{1}{2 m}}} \frac{(\sqrt{N(N-2)} \epsilon)^{N}}{|x|^{2 N}} d x \\
& =2[N(N-2)]^{\frac{N}{2}} \epsilon^{N} \int_{\mathbb{R}^{N} \backslash B_{\frac{1}{2 m}}} \frac{1}{|x|^{2 N}} d x .
\end{aligned}
$$

Como

$$
\int_{\mathbb{R}^{N} \backslash B_{\frac{1}{2 m}}} \frac{1}{|x|^{2 N}} d x=\frac{\omega_{N}}{N}(2 m)^{N},
$$

temos que $\left.||\left|\Phi_{\epsilon, m}\right|\right|_{2^{*}} ^{2^{*}}-S^{\frac{N}{2}} \mid \leq D_{2}(N)(\epsilon m)^{N}$, provando o item (b).

- Prova do item (c):

Como $\epsilon m \rightarrow 0$, assumiremos que $\epsilon m<\frac{1}{2}$. Desse modo, faremos a seguinte estimativa

$$
\begin{aligned}
\left\|\Phi_{\epsilon, m}\right\|_{s}^{s} & =\int_{\Omega}\left|\xi_{m}(x) \Phi_{\epsilon}(x)\right|^{s} d x \\
& =\int_{B_{\frac{1}{2 m}}}\left|\Phi_{\epsilon}(x)\right|^{s} d x+\int_{B_{\frac{1}{m} \backslash B_{\frac{1}{2 m}}}}\left|\xi_{m}(x) \Phi_{\epsilon}(x)\right|^{s} d x \\
& \geq \int_{B_{\frac{1}{2 m}}}\left|\Phi_{\epsilon}(x)\right|^{s} d x
\end{aligned}
$$




$$
\begin{aligned}
& =\int_{B_{\frac{1}{2 m}}}\left(\frac{\sqrt{N(N-2)} \epsilon}{\epsilon^{2}+|x|^{2}}\right)^{\frac{N-2}{2} s} d x \\
& \geq \int_{B_{\epsilon}}\left(\frac{\sqrt{N(N-2) \epsilon}}{\epsilon^{2}+|x|^{2}}\right)^{\frac{N-2}{2} s} d x .
\end{aligned}
$$

Como $|x| \leq \epsilon$,

$$
\begin{aligned}
\left\|\Phi_{\epsilon, m}\right\|_{s}^{s} & \geq[N(N-2)]^{\frac{N-2}{4} s} \epsilon^{\frac{N-2}{2} s} \int_{B_{\epsilon}} \frac{1}{\left(2 \epsilon^{2}\right)^{\frac{N-2}{2} s}} d x \\
& =\left(\frac{N(N-2)}{4}\right)^{\frac{N-2}{4} s} \frac{\omega_{N}}{N} \epsilon^{-\frac{N-2}{2} s} \epsilon^{N} .
\end{aligned}
$$

Temos, então, que $\left\|\Phi_{\epsilon, m}\right\|_{s}^{s} \geq D_{3}(N, s) \epsilon^{N-\frac{N-2}{2} s}$, provando o item (c).

- Prova do item (d):

$$
\begin{aligned}
\left\|\Phi_{\epsilon, m}\right\|_{2}^{2} & =\int_{\Omega}\left|\xi_{m}(x) \Phi_{\epsilon}(x)\right|^{2} d x \\
& \leq \int_{B_{\frac{1}{m}}}\left|\Phi_{\epsilon}(x)\right|^{2} d x \\
& =\int_{B_{\frac{1}{m}}}\left(\frac{\sqrt{N(N-2)} \epsilon}{\epsilon^{2}+|x|^{2}}\right)^{N-2} d x .
\end{aligned}
$$

Considerando $x=\epsilon w$, obtemos

$$
\left\|\Phi_{\epsilon, m}\right\|_{2}^{2} \leq[N(N-2)]^{\frac{N-2}{2}} \epsilon^{2} \int_{B_{\frac{1}{\epsilon m}}} \frac{1}{\left(1+|w|^{2}\right)^{N-2}} d w .
$$


Lembrando que $\epsilon m \rightarrow 0$, podemos assumir $\epsilon m<1$, assim

$$
\begin{aligned}
\left\|\Phi_{\epsilon, m}\right\|_{2}^{2} & \leq[N(N-2)]^{\frac{N-2}{2}} \epsilon^{2}\left[\int_{B_{1}} \frac{1}{\left(1+|w|^{2}\right)^{N-2}} d w+\int_{B_{\frac{1}{\epsilon m}} \backslash B_{1}} \frac{1}{\left(1+|w|^{2}\right)^{N-2}} d w\right] \\
& \leq[N(N-2)]^{\frac{N-2}{2}} \epsilon^{2}\left[\frac{\omega_{N}}{N}+\int_{\left.B_{\frac{1}{\epsilon m} \backslash B_{1}} \frac{1}{|w|^{2 N-4}} d w\right]}\right] \\
& =[N(N-2)]^{\frac{N-2}{2}} \epsilon^{2}\left[\frac{\omega_{N}}{N}+\omega_{N} \int_{1}^{\frac{1}{\epsilon m}} \frac{1}{r^{N-3}} d r\right] .
\end{aligned}
$$

Como

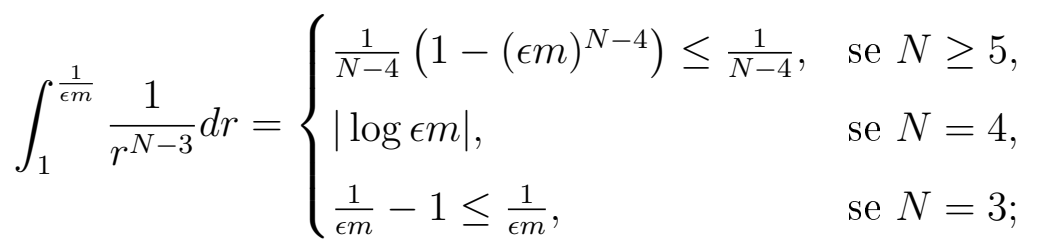

então

$$
\left\|\Phi_{\epsilon, m}\right\|_{2}^{2} \leq \begin{cases}D(N) \epsilon^{2}, & \text { se } N \geq 5 \\ D(4) \epsilon^{2}|\log (\epsilon m)|, & \text { se } N=4 \\ D(3) \frac{\epsilon}{m}, & \text { se } N=3\end{cases}
$$

Assim fica provado o item (d).

\section{Observação 5.6.}

Devemos observar que, para $\epsilon m<1$, vale $|\log (\epsilon m)|=\log \left(\frac{1}{\epsilon m}\right) \leq \frac{1}{\epsilon m}$, logo $\left\|\Phi_{\epsilon, m}\right\|_{2}^{2} \rightarrow 0$, nas hipóteses do Lema 5.5, para todo $N \geq 3$.

\subsection{As autofunções aproximadas}

Ao longo da demonstração dos Teoremas 5.1 e 5.2 utilizaremos as autofunções do laplaciano em $E,\left\{\phi_{k}\right\}_{k \in \mathbb{N}}$. Como o intuito é obtermos estimativas melhores que as encontradas em [DFJ99] "cavaremos um buraco" também nas autofunções; para isso, consideramos 
$\zeta_{m} \in C^{\infty}(\bar{\Omega})$ tal que

$$
\begin{aligned}
& 0 \leq \zeta_{m} \leq 1, \\
& \zeta_{m}(x)= \begin{cases}0, & \text { se } x \in B_{\frac{2}{m}}\left(x_{m}\right) \\
1, & \text { se } x \in \Omega \backslash B_{\frac{3}{m}}\left(x_{m}\right),\end{cases} \\
& \left|\nabla \zeta_{m}(x)\right| \leq 4 m .
\end{aligned}
$$

onde os pontos $x_{m}$ são os mesmos escolhidos no início da seção 5.2. Definimos, então, as "autofunções aproximadas" por $\phi_{k}^{m}(x)=\zeta_{m}(x) \phi_{k}(x)$.

Precisamos do seguinte Lema.

Lema 5.7. Para $m$ suficientemente grande, o conjunto $\left\{\phi_{k}^{m}\right\}_{k \in \mathbb{N}}$ é linearmente independente.

Demonstração. O conjunto $\left\{\phi_{k}\right\}_{k \in \mathbb{N}}$ é linearmente independente, uma vez que $\left\{\phi_{k}\right\}_{k \in \mathbb{N}}$ constitui um conjunto ortogonal em $E$. Para $m$ suficientemente grande, como $\zeta_{m} \equiv 1$ em $\Omega \backslash B_{\frac{3}{m}}\left(x_{m}\right)$, existe um aberto $\omega \subset \Omega \backslash B_{\frac{3}{m}}\left(x_{m}\right)$ no qual $\phi_{i}=\phi_{i}^{m}$ para todo $i \in \mathbb{N}$.

Suponhamos que $u=\sum_{i=1}^{n} \beta_{i} \phi_{i}^{m}=0$.

Observe que se os $\phi_{i}$ que aparecem na soma correspondem todos ao mesmo autovalor $\lambda$, então $v=\sum_{i=1}^{n} \beta_{i} \phi_{i}$ é solução de $-\Delta v=\lambda v$ em $\Omega$, identicamente nula em $\omega$ : pelo Corolário 1.1 de [GL87] uma tal solução é necessariamente identicamente zero em todo $\Omega$, e pela independência linear de $\left\{\phi_{k}\right\}_{k \in \mathbb{N}}$ isso implica que todos os coeficientes são zero.

Sem perda de generalidade podemos então considerar agora $u=\sum_{i=1}^{n} \beta_{i} \phi_{i}^{m}=0$ sendo todas as $\phi_{i}$ que aparecem na soma correspondentes a autovalores $\lambda_{i}$ distintos.

É claro que se $n=1$ isso implica $\beta_{1}=0$.

Suponhamos então que, para certo $n \in \mathbb{N}$, valha a propriedade

$$
\text { (H) se } \sum_{i=1}^{n} \beta_{i} \phi_{i}^{m}=0 \text { em } \omega \text { então todos os coeficientes são zero. }
$$

Consideremos então $u=\sum_{i=1}^{n+1} \beta_{i} \phi_{i}^{m}=0 . \operatorname{Em} \omega, u=\sum_{i=1}^{n+1} \beta_{i} \phi_{i}=0$ e logo vale

$$
-\Delta u-\lambda_{n+1} u=0=\sum_{i=1}^{n+1} \beta_{i}\left(\lambda_{i}-\lambda_{n+1}\right) \phi_{i}=\sum_{i=1}^{n+1} \beta_{i}\left(\lambda_{i}-\lambda_{n+1}\right) \phi_{i}^{m},
$$

isto é $\sum_{i=1}^{n} \beta_{i}\left(\lambda_{i}-\lambda_{n+1}\right) \phi_{i}^{m}=0$ em $\omega$ : sendo uma soma de $n$ elementos deduzimos, por 
hipótese de indução, que $\beta_{i}=0$ para $i=1, . . n$. Mas então também $\beta_{n+1}=0$, o que prova que a propriedade $(H)$ vale para todo $n \in \mathbb{N}$.

Consideraremos

$$
W_{m, k}:=\left\langle\phi_{i}^{m}\right\rangle_{i=1}^{k}
$$

observando que, pelo lema acima, $\operatorname{dim} W_{m, k}=k$. Recordemos que $W_{k}=\left\langle\phi_{i}\right\rangle_{i=1}^{k}$.

O Lema a seguir nos mostra, em particular, que quando $m \rightarrow \infty$ as funções $\phi_{k}^{m}$ tem propriedades análogas às das funções $\phi_{k}$. A demonstração é baseada no Lema 2 de [GR97].

\section{Lema 5.8.}

Para cada $k \in \mathbb{N}, \phi_{k}^{m} \underset{m \rightarrow \infty}{\longrightarrow} \phi_{k}$ em E. Além disso, no espaço $W_{m, k}$ temos

$$
\max \left\{\|u\|_{E}^{2}: u \in W_{m, k},\|u\|_{2}=1\right\} \leq \lambda_{k}+C_{k}(N) m^{-N},
$$

e fixados $i, j \in \mathbb{N}$

$$
\left|\int_{\Omega} \nabla \phi_{i}^{m} \cdot \nabla \phi_{j}^{m} d x-\delta_{i, j}\right| \leq C_{i j}(N) m^{-N}
$$

\section{Prova.}

De modo idêntico ao que foi feito para a função $u_{t}$, no início da demonstração do Lema 5.3, podemos dizer que existe $c_{k}>0$ tal que para todo $x \in B_{\frac{4}{m}}\left(x_{m}\right)$ vale

$$
\left|\phi_{k}(x)\right| \leq \frac{c_{k}}{m}
$$


uma vez que $\phi_{k} \in C^{\infty}(\bar{\Omega}) \cap E$. Disso obtemos

$$
\begin{aligned}
\left\|\phi_{k}^{m}-\phi_{k}\right\|_{E}^{2}= & \int_{\Omega}\left|\nabla\left(\phi_{k}^{m}-\phi_{k}\right)\right|^{2} d x \\
= & \int_{\Omega}\left|\phi_{k} \nabla \zeta_{m}+\left(\zeta_{m}-1\right) \nabla \phi_{k}\right|^{2} d x \\
= & \int_{\Omega}\left|\phi_{k}\right|^{2}\left|\nabla \zeta_{m}\right|^{2} d x+2 \int_{\Omega}\left(\zeta_{m}-1\right) \phi_{k} \nabla \phi_{k} \cdot \nabla \zeta_{m} d x+\int_{\Omega}\left|\zeta_{m}-1\right|^{2}\left|\nabla \phi_{k}\right|^{2} d x \\
= & \int_{B_{\frac{3}{m}} \backslash B_{\frac{2}{m}}}\left|\phi_{k}\right|^{2}\left|\nabla \zeta_{m}\right|^{2} d x+2 \int_{B_{\frac{3}{m}} \backslash B_{\frac{2}{m}}}\left(\zeta_{m}-1\right) \phi_{k} \nabla \phi_{k} \cdot \nabla \zeta_{m} d x \\
& +\int_{B_{\frac{3}{m}}}\left|\zeta_{m}-1\right|^{2}\left|\nabla \phi_{k}\right|^{2} d x \\
\leq & \int_{B_{\frac{3}{m}} \backslash B_{\frac{2}{m}}}\left|\phi_{k}\right|^{2}\left|\nabla \zeta_{m}\right|^{2} d x+2 \int_{B_{\frac{3}{m}} \backslash B_{\frac{2}{m}}}\left|\zeta_{m}-1 \| \phi_{k}\right|\left|\nabla \phi_{k}\right|\left|\nabla \zeta_{m}\right| d x \\
& +\int_{B_{\frac{3}{m}}}\left|\zeta_{m}-1\right|^{2}\left|\nabla \phi_{k}\right|^{2} d x \\
\leq & \frac{\omega_{N}}{N}\left(\frac{3^{N}-2^{N}}{m^{N}} \frac{c_{k}^{2}}{m^{2}} 16 m^{2}+2 \frac{3^{N}-2^{N}}{m^{N}} \frac{c_{k}}{m} 4 m\left\|\nabla \phi_{k}\right\|\left\|_{\infty}+\frac{3^{N}}{m^{N}}\right\| \nabla \phi_{k} \|_{\infty}^{2}\right) \\
= & C(N, k) m^{-N} .
\end{aligned}
$$

Com isso mostramos a convergência em $E$ de $\phi_{k}^{m}$ a $\phi_{k}$.

Para provar (5.12) consideraremos $S=\left\{u \in E:\|u\|_{2}=1\right\}, S_{k}=W_{k} \cap S$ e $S_{m, k}=$ $W_{m . k} \cap S$. Pelo item (i) do TEOREMA DE MERGULHO DE SOBOLEV a inclusão $i: E \rightarrow$ $L^{2}(\Omega)$ é contínua. Considerando $\theta: L^{2}(\Omega) \rightarrow \mathbb{R}$ a aplicação norma, temos que a aplicação $\Theta: E \rightarrow \mathbb{R}$ dada por $\Theta=\theta \circ i$ é contínua, logo os conjuntos $S_{k}$ e $S_{m, k}$ são fechados em $E$ uma vez que $S=\Theta^{-1}(\{1\})$. Como as normas $\|\cdot\|_{E}$ e $\|\cdot\|_{2}$ são equivalentes em $W_{k}$ e $W_{m, k}$, temos que $S_{k}$ e $S_{m, k}$ são conjuntos compactos. Em particular, da continuidade da norma de $E$, segue que existe $u_{m} \in S_{m, k}$ tal que

$$
\left\|u_{m}\right\|_{E}^{2}=\max _{u \in S_{m, k}}\|u\|_{E}^{2}
$$

Definimos a aplicação $A_{m}: S_{k} \rightarrow S_{m, k}$ por

$$
A_{m}(\widetilde{u})=\frac{1}{\left\|\zeta_{m} \widetilde{u}\right\|_{2}} \zeta_{m} \widetilde{u}
$$


Se considerarmos $\widetilde{u}=\sum_{1=1}^{k} \alpha_{i} \phi_{i}$, temos, de modo equivalente, que

$$
A_{m}(\widetilde{u})=\frac{1}{\left\|\sum_{i=1}^{k} \alpha_{i} \phi_{i}^{m}\right\|_{2}} \sum_{i=1}^{k} \alpha_{i} \phi_{i}^{m}
$$

Como $\left\{\phi_{i}^{m}\right\}_{i=1}^{k}$ constitui um conjunto linearmente independente, temos que $A_{m}$ é bijetiva; além disso, é fácil verificar que sua inversa é a aplicação $B_{m}: S_{m, k} \rightarrow S_{k}$ dada por

$$
B_{m}(u)=B_{m}\left(\sum_{i=1}^{k} \beta_{i} \phi_{i}^{m}\right)=\frac{1}{\left\|\sum_{i=1}^{k} \beta_{i} \phi_{i}\right\|_{2}} \sum_{i=1}^{k} \beta_{i} \phi_{i} .
$$

Podemos agora dizer que existe $\widetilde{u}_{m} \in S_{k}$ tal que

$$
u_{m}=A_{m}\left(\widetilde{u}_{m}\right)=\frac{1}{\left\|\zeta_{m} \widetilde{u}_{m}\right\|_{2}} \zeta_{m} \widetilde{u}_{m}
$$

Seja $\widetilde{u} \in S_{k}$ qualquer e escreva $\widetilde{u}=\sum_{i=1}^{k} \alpha_{i} \phi_{i}$. Dado $x \in B_{\frac{4}{m}}\left(x_{m}\right)$, de (5.14) obtemos

$$
|\widetilde{u}(x)| \leq \sum_{i=1}^{k}\left|\alpha_{i}\right|\left|\phi_{i}(x)\right| \leq \sum_{i=1}^{k}\left|\alpha_{i}\right| \frac{c_{i}}{m} \leq \frac{\bar{C}_{k}}{m} \sum_{i=1}^{k}\left|\alpha_{i}\right| ;
$$

onde $\bar{C}_{k}=\max _{1 \leq i \leq k}\left\{c_{i}\right\}$. Como $\|\widetilde{u}\|_{2}=1$ e $\operatorname{dim} W_{k}<\infty$, deve existir $\widetilde{C}_{k}>0$ tal que

$$
|\widetilde{u}(x)| \leq \frac{\widetilde{C}_{k}}{m}, \quad \forall x \in B_{\frac{4}{m}}\left(x_{m}\right) \quad \text { e } \quad \forall \widetilde{u} \in S_{k}
$$

Segue daí que se $\widetilde{u} \in S_{k}$, então

$$
\begin{aligned}
1 & =\frac{1}{\left\|\zeta_{m} \widetilde{u}\right\|_{2}^{2}} \int_{\Omega}\left|\zeta_{m} \widetilde{u}\right|^{2} \\
& =\frac{1}{\left\|\zeta_{m} \widetilde{u}\right\|_{2}^{2}}\left[\int_{\Omega} \widetilde{u}^{2}-\int_{B_{\frac{3}{m}}} \widetilde{u}^{2}+\int_{B_{\frac{3}{m}} \backslash B_{\frac{2}{m}}}\left|\zeta_{m} \widetilde{u}\right|^{2}\right] \\
& \geq \frac{1}{\left\|\zeta_{m} \widetilde{u}\right\|_{2}^{2}}\left[1-3^{N} \widetilde{C}_{k}^{2} \frac{\omega_{N}}{N} m^{-2-N}\right] \\
& =\frac{1}{\left\|\zeta_{m} \widetilde{u}\right\|_{2}^{2}}\left[1-C(k, N) m^{-2-N}\right]
\end{aligned}
$$


o que nos dá

$$
\frac{1}{\left\|\zeta_{m} \widetilde{u}\right\|_{2}^{2}} \leq 1+C m^{-2-N} \quad \forall \widetilde{u} \in S_{k}
$$

Assim, dado $u \in S_{m, k}$ e lembrando que $\left\|\nabla \zeta_{m}\right\|_{\infty} \leq 4 m$, temos

$$
\begin{aligned}
\|u\|_{E}^{2} & \leq\left\|u_{m}\right\|_{E}^{2} \\
& =\frac{1}{\left\|\zeta_{m} \widetilde{u}_{m}\right\|_{2}^{2}}\left\|\zeta_{m} \widetilde{u}_{m}\right\|_{E}^{2}
\end{aligned}
$$

Como $\widetilde{u}_{m} \in S_{k}$, de (5.16) temos

$$
\begin{aligned}
& \|u\|_{E}^{2} \leq\left(1+C m^{-2-N}\right) \int_{\Omega}\left|\nabla\left(\zeta_{m} \widetilde{u}_{m}\right)\right|^{2} d x \\
& =\left(1+C m^{-2-N}\right)\left[\int_{\Omega} \zeta_{m}^{2}\left|\nabla \widetilde{u}_{m}\right|^{2} d x+2 \int_{\Omega} \zeta_{m} \widetilde{u}_{m} \nabla \widetilde{u}_{m} \cdot \nabla \zeta_{m} d x+\int_{\Omega} \widetilde{u}_{m}^{2}\left|\nabla \zeta_{m}\right|^{2} d x\right] \\
& \leq\left(1+C m^{-2-N}\right)\left[\left\|\widetilde{u}_{m}\right\|_{E}^{2}+8 m \int_{B_{\frac{3}{m} \backslash B_{\frac{2}{m}}}} \widetilde{u}_{m}\left|\nabla \widetilde{u}_{m}\right| d x+16 m^{2} \int_{B_{\frac{3}{m} \backslash B_{\frac{2}{m}}}} \widetilde{u}_{m}^{2} d x\right] .
\end{aligned}
$$

Como nas regiões de integração acima $\widetilde{u}_{m}$ satisfaz (5.15), temos, então

$$
\begin{aligned}
\|u\|_{E}^{2} \leq & \left(1+C m^{-2-N}\right)\left[\left\|\widetilde{u}_{m}\right\|_{E}^{2}+8 m \frac{\widetilde{C}_{k}}{m}\left\|\nabla \widetilde{u}_{m}\right\|_{\infty}\left(3^{N}-2^{N}\right) \frac{\omega_{N}}{N} m^{-N}\right. \\
& \left.+16 m^{2} \frac{\widetilde{C}_{k}^{2}}{m^{2}}\left(3^{N}-2^{N}\right) \frac{\omega_{N}}{N} m^{-N}\right]
\end{aligned}
$$

Como existe uma constante positiva, que também denotaremos por $\widetilde{C}_{k}$, tal que $\|\nabla \widetilde{u}\|_{\infty} \leq \widetilde{C}_{k}$ para todo $\widetilde{u} \in S_{k}$, temos

$$
\begin{aligned}
\|u\|_{E}^{2} \leq & \left(1+C m^{-2-N}\right)\left[\left\|\widetilde{u}_{m}\right\|_{E}^{2}+8 \widetilde{C}_{k}^{2}\left(3^{N}-2^{N}\right) \frac{\omega_{N}}{N} m^{-N}\right. \\
& \left.+16 \widetilde{C}_{k}^{2}\left(3^{N}-2^{N}\right) \frac{\omega_{N}}{N} m^{-N}\right] .
\end{aligned}
$$

Usando o item (a) da Proposição A.1 e lembrando que $\left\|\widetilde{u}_{m}\right\|_{2}=1$, dado $u \in S_{m, k}$ obtemos

$$
\begin{aligned}
\|u\|_{E}^{2} & \leq\left(1+C m^{-2-N}\right)\left[\lambda_{k}+\widehat{C}(k, N) m^{-N}\right] \\
& \leq \lambda_{k}+C(k, N) m^{-N} .
\end{aligned}
$$


Por fim, provaremos (5.13). Lembrando que $\left\|\nabla \zeta_{m}\right\|_{\infty} \leq 4 m$

$$
\begin{aligned}
& \left|\int_{\Omega} \nabla \phi_{i}^{m} \cdot \nabla \phi_{j}^{m} d x-\delta_{i j}\right| \\
& =\mid \int_{\Omega} \zeta_{m}^{2} \nabla \phi_{i} \cdot \nabla \phi_{j} d x+\int_{\Omega} \zeta_{m} \phi_{i} \nabla \phi_{j} \cdot \nabla \zeta_{m} d x \\
& +\int_{\Omega} \zeta_{m} \phi_{j} \nabla \phi_{i} \cdot \nabla \zeta_{m} d x+\int_{\Omega} \phi_{i} \phi_{j}\left|\nabla \zeta_{m}\right|^{2} d x-\delta_{i j} \mid \\
& =\mid \int_{\Omega \backslash B_{\frac{3}{m}}} \nabla \phi_{i} \cdot \nabla \phi_{j} d x+\int_{B_{\frac{3}{m} \backslash B_{\frac{2}{m}}}} \zeta_{m}^{2} \nabla \phi_{i} \cdot \nabla \phi_{j} d x+\int_{B_{\frac{3}{m} \backslash B_{\frac{2}{m}}}} \zeta_{m} \phi_{i} \nabla \phi_{j} \cdot \nabla \zeta_{m} d x \\
& +\int_{B_{\frac{3}{m} \backslash B_{\frac{2}{m}}}} \zeta_{m} \phi_{j} \nabla \phi_{i} \cdot \nabla \zeta_{m} d x+\int_{B_{\frac{3}{m} \backslash B_{\frac{2}{m}}}} \phi_{i} \phi_{j}\left|\nabla \zeta_{m}\right|^{2} d x-\int_{\Omega} \nabla \phi_{i}^{m} \cdot \nabla \phi_{j}^{m} d x \mid \\
& =\mid-\int_{B_{\frac{3}{m}}} \nabla \phi_{i} \cdot \nabla \phi_{j} d x+\int_{B_{\frac{3}{m} \backslash B_{\frac{2}{m}}}} \zeta_{m}^{2} \nabla \phi_{i} \cdot \nabla \phi_{j} d x+\int_{B_{\frac{3}{m} \backslash B_{\frac{2}{m}}}} \zeta_{m} \phi_{i} \nabla \phi_{j} \cdot \nabla \zeta_{m} d x \\
& +\int_{B_{\frac{3}{m} \backslash B_{\frac{2}{m}}}} \zeta_{m} \phi_{j} \nabla \phi_{i} \cdot \nabla \zeta_{m} d x+\int_{B_{\frac{3}{m}} \backslash B_{\frac{2}{m}}} \phi_{i} \phi_{j}\left|\nabla \zeta_{m}\right|^{2} d x \mid \\
& \leq \int_{B_{\frac{3}{m}}}\left|\nabla \phi_{i}\right|\left|\nabla \phi_{j}\right| d x+\int_{B_{\frac{3}{m} \backslash B_{\frac{2}{m}}}}\left|\nabla \phi_{i}\right|\left|\nabla \phi_{j}\right| d x+4 m \int_{B_{\frac{3}{m} \backslash B_{\frac{2}{m}}}}\left|\phi_{i}\right|\left|\nabla \phi_{j}\right| d x \\
& +4 m \int_{B_{\frac{3}{m} \backslash B_{\frac{2}{m}}}}\left|\phi_{j}\right|\left|\nabla \phi_{i}\right| d x+16 m^{2} \int_{B_{\frac{3}{m} \backslash B_{\frac{2}{m}}}}\left|\phi_{i}\right|\left|\phi_{j}\right| d x .
\end{aligned}
$$

Como nas regiões de integração acima vale (5.14),

$$
\begin{aligned}
\left|\int_{\Omega} \nabla \phi_{i}^{m} \cdot \nabla \phi_{j}^{m} d x-\delta_{i j}\right| \leq & 3^{N} \mid\left\|\nabla \phi_{i}\right\|_{\infty}\left\|\nabla \phi_{j}\right\|_{\infty} \frac{\omega_{N}}{N} m^{-N} \\
& +\left(3^{N}-2^{N}\right)\left\|\nabla \phi_{i}\right\|_{\infty}\left\|\nabla \phi_{j}\right\|_{\infty} \frac{\omega_{N}}{N} m^{-N} \\
& +4 m \frac{c_{i}}{m}\left\|\nabla \phi_{j}\right\|_{\infty}\left(3^{N}-2^{N}\right) c_{i} \frac{\omega_{N}}{N} m^{-N} \\
& +4 m \frac{c_{j}}{m}\left\|\nabla \phi_{i}\right\|_{\infty}\left(3^{N}-2^{N}\right) \frac{\omega_{N}}{N} m^{-N} \\
& +16 m^{2} \frac{c_{i} c_{j}}{m^{2}}\left(3^{N}-2^{N}\right) \frac{\omega_{N}}{N} m^{-N} \\
= & C(i, j, N) m^{-N}
\end{aligned}
$$




\section{Observação 5.9.}

Observe que para todo $u \in W_{m, k}$, se considerarmos $\widetilde{u}=\frac{u}{\|u\|_{2}}$, então $\widetilde{u} \in S_{m, k}$; logo de (5.12) temos que $\|\widetilde{u}\|_{E}^{2} \leq \lambda_{k}+c_{k} m^{-N}$ o que nos dá

$$
\|u\|_{E}^{2} \leq\left(\lambda_{k}+c_{k} m^{-N}\right)\|u\|_{2}^{2}
$$

\section{Observação 5.10 .}

Fixando $k \in \mathbb{N}$, já sabemos que os conjuntos $W_{m, k}$ e $W_{k}$ têm a mesma dimensão e que $E=W_{k} \oplus X_{k}$. Em vista das convergência provadas no Lema 5.8, podemos afirmar que, para $m$ suficientemente grande, $W_{m, k} \cap X_{k}=\{0\}$, logo vale também a decomposição direta (mas, neste caso, não ortogonal) $E=W_{m, k} \oplus X_{k}$.

\subsection{Observação sobre as funções cortadas}

Antes de prosseguirmos, é importante observar que as funções $\eta_{m}, \zeta_{m}, \xi_{m}$ foram escolhidas apropriadamente como mostra a tabela abaixo:

\begin{tabular}{|c|c|c|c|c|c|l|}
\hline & $B_{\frac{1}{2 m}}$ & $B_{\frac{1}{m}} \backslash B_{\frac{1}{2 m}}$ & $B_{\frac{2}{m}} \backslash B_{\frac{1}{m}}$ & $B_{\frac{3}{m}} \backslash B_{\frac{2}{m}}$ & $\Omega \backslash B_{\frac{3}{m}}$ & Funções cortadas \\
\hline$\eta_{m}$ & 0 & 0 & $0-1$ & 1 & 1 & $u_{t}^{m}=\eta_{m} u_{t}$ \\
$\zeta_{m}$ & 0 & 0 & 0 & $0-1$ & 1 & $\phi_{i}^{m}=\zeta_{m} \phi_{i}$ \\
$\xi_{m}$ & 1 & $0-1$ & 0 & 0 & 0 & $\Phi_{\epsilon, m}=\xi_{m} \Phi_{\epsilon}$ \\
\hline
\end{tabular}

Nessa tabela, 0 - 1 significa que a função passa entre os valores 0 e 1 , por consequência o gradiente da função é não nulo na região correspondente, mas é zero nas outras.

Fixado $m \in \mathbb{N}$, sejam $w \in W_{m, k}$ e $\Phi_{\epsilon, m}$ dados. De acordo com a tabela, temos:

(a) Como o suporte de $w$ e $\Phi_{\epsilon, m}$ são disjuntos, $\Phi_{\epsilon, m} \in W_{m, k}^{\perp}$, logo

$$
\begin{gathered}
\int_{\Omega}\left|\nabla\left(w+\Phi_{\epsilon, m}\right)\right|^{2} d x=\int_{\Omega}|\nabla w|^{2} d x+\left|\nabla\left(\Phi_{\epsilon, m}\right)\right|^{2} d x, \\
\int_{\Omega}\left|\left(w+\Phi_{\epsilon, m}\right)\right|^{2} d x=\int_{\Omega}|w|^{2} d x+\left|\Phi_{\epsilon, m}\right|^{2} d x .
\end{gathered}
$$


(b) Em $B_{\frac{1}{m}}$ temos $w=0$ e $u_{t}^{m}=0$, enquanto em $\Omega \backslash B_{\frac{1}{m}}$ temos $\Phi_{\epsilon, m}=0$, então

$$
\int_{\Omega}\left(w+\Phi_{\epsilon, m}+u_{t}^{m}\right)_{+}^{2^{*}} d x=\int_{B_{\frac{1}{m}}}\left(\Phi_{\epsilon, m}+u_{t}^{m}\right)_{+}^{2^{*}} d x+\int_{\Omega \backslash B_{\frac{1}{m}}}\left(w+u_{t}^{m}\right)_{+}^{2^{*}} d x
$$

mas como ambas as funções nas integrais são nulas no resto de $\Omega$ (lembre que $u_{t}^{m} \leq 0$ ), podemos reescrever como

$$
\int_{\Omega}\left(w+\Phi_{\epsilon, m}+u_{t}^{m}\right)_{+}^{2^{*}} d x=\int_{\Omega}\left(\Phi_{\epsilon, m}+u_{t}^{m}\right)_{+}^{2^{*}} d x+\int_{\Omega}\left(w+u_{t}^{m}\right)_{+}^{2^{*}} d x
$$

da mesma maneira

$$
\begin{aligned}
\int_{\Omega} G\left(x,\left(w+\Phi_{\epsilon, m}+u_{t}^{m}\right)_{+}\right) d x & =\int_{B_{\frac{1}{m}}} G\left(x,\left(\Phi_{\epsilon, m}+u_{t}^{m}\right)_{+}\right) d x+\int_{\Omega \backslash B_{\frac{1}{m}}} G\left(x,\left(w+u_{t}^{m}\right)_{+}\right) d x \\
& =\int_{\Omega} G\left(x,\left(\Phi_{\epsilon, m}+u_{t}^{m}\right)_{+}\right) d x+\int_{\Omega} G\left(x,\left(w+u_{t}^{m}\right)_{+}\right) d x .
\end{aligned}
$$

Segue dos itens (a) e (b) acima e do fato de $F$ e $F_{m}$ serem lineares, que para qualquer $v=w+s \Phi_{\epsilon, m} \in W_{m, k} \oplus \mathbb{R} \Phi_{\epsilon, m}$, vale

$$
I_{m}(v)=I_{m}\left(w+s \Phi_{\epsilon, m}\right)=I_{m}(w)+I_{m}\left(s \Phi_{\epsilon, m}\right) .
$$

\subsection{A estrutura de enlace do funcional}

Nosso objetivo, a partir daqui, é verificar se existem certos valores de $m \in \mathbb{N}$ para os quais o funcional $I_{m}$, dado por (5.3), possua um ponto crítico.

De acordo com o exposto no capítulo 2, para mostrarmos que o funcional $I_{m}$ possui um ponto crítico, devemos investigar se $I_{m}$ satisfaz a condição $(P S)$ ou $(P S)_{c}$ e se $I_{m}$ satisfaz as hipóteses do TEOREMA DE ENLACE. Esse foi o procedimento utilizado no capítulo 4. No entanto, procederemos aqui de um modo um pouco diferente, pelo fato de estarmos considerando o caso crítico. Ainda mostraremos que $I_{m}$ satisfaz as hipóteses do 
TEOREMA DE ENLACE: isso, sem a condição $(P S)$, nos garante apenas a existência de uma sequência $(P S)$ para $I_{m}$ num nível $c_{m}$; denotaremos tal sequência por $\left\{v_{n}\right\}_{n \in \mathbb{N}}$ sem indicar a dependência com respeito a $m$ a fim de não sobrecarregar a notação.

Nas seções 5.8, 5.9 e 5.10 nos preocuparemos em mostrar que tal sequência converge fracamente a um elemento $v_{0}$ (onde a dependência com respeito a $m$ também será omitida) e que, para $m$ grande, $v_{0}$ é ponto crítico de $I_{m}$ distinto de $u_{t}-u_{t}^{m}$, ou seja, $v_{0}$ será a segunda solução que procuramos

Nesta seção mostraremos que para certos valores de $m \in \mathbb{N}$ o funcional $I_{m}$ tem estrutura de enlace, ou seja, mostraremos que existem subconjuntos fechados $S$ e $Q$ de $E$ que enlaçam e constantes $\beta<\alpha$ para os quais são válidas as hipóteses do TEOREMA DE ENLACE para o funcional $I_{m}$. Para isso, consideraremos os subespaços $W_{m, k}$, definido em (5.11), e $X_{k}$; lembrando que $X_{k}=W_{k}^{\perp}$ e $W_{k}=\left\langle\phi_{i}\right\rangle_{i=1}^{k}$. Lembre que vale a decomposição $E=W_{m, k} \oplus X_{k}$ (veja a observação 5.10).

Dado $\rho>0$ considere $B_{\rho}=\left\{v \in E:\|v\|_{E}<\rho\right\}$. Sejam $R, \epsilon>0$ e $m \in \mathbb{N}$ fixados, e consideremos os subconjuntos de $E$

$$
\begin{aligned}
& S_{\rho}:=\partial B_{\rho} \cap X_{k} \\
& Q_{R, m}^{\epsilon}:=\left(B_{R} \cap W_{m, k}\right) \oplus\left\{s \Phi_{\epsilon, m}: s \in[0, R]\right\} .
\end{aligned}
$$

Lembrando que $\Phi_{\epsilon, m} \in W_{m, k}^{\perp}$, é possível mostrar que $Q_{R, m}^{\epsilon}$ é homeomorfo à bola unitária fechada de $\mathbb{R}^{k+1}$. Neste caso, temos que $\partial Q_{R, m}^{\epsilon}=\cup_{i=1}^{3} \Gamma_{i}$ onde

$$
\begin{aligned}
& \Gamma_{1}=W_{m, k} \cap \bar{B}_{R}, \\
& \Gamma_{2}=\left\{v \in E: v=w+s \Phi_{\epsilon, m}, w \in W_{m, k},\|w\|_{E}=R \text { e } s \in[0, R]\right\}, \\
& \Gamma_{3}=\left\{v \in E: v=w+R \Phi_{\epsilon, m}, w \in W_{m, k} \cap \bar{B}_{R}\right\} .
\end{aligned}
$$

Observe que a única diferença entre os conjuntos em (5.19) com relação aos conjuntos obtidos nos Lemas 4.4 e 4.5 do capítulo 4 , é que $\Phi_{\epsilon, m} \notin X_{k}$. No entanto, se escolhermos $R, \epsilon>0$ e $m \in \mathbb{N}$ de modo que $R>\rho$ e $R\left\|\phi_{\epsilon, m}\right\|_{E}>\rho$, então $S_{\rho}$ intersecta $Q_{R, m}^{\epsilon} \backslash \partial Q_{R, m}^{\epsilon}$ e com isso ainda é possível mostrar que os subconjuntos $S_{\rho}$ e $Q_{R, m}^{\epsilon}$ enlaçam, seguindo os mesmos passos do exemplo $8.3 \mathrm{em}$ [Str08].

\section{Lema 5.11.}

Se $m \in \mathbb{N}$ é suficientemente grande, então existem números $\rho, \alpha>0$, independentes de $m e$ 
$\epsilon$, tais que

$$
\left.I\right|_{S_{\rho}} \geq \alpha
$$

\section{Prova.}

Seja $v \in X_{k}$. Utilizando o item (b) da Proposição A.1, verifica-se facilmente que

$$
\frac{1}{2} \int_{\Omega}\left(|\nabla v|^{2}-\lambda v^{2}\right) \geq \frac{1}{2}\left(1-\frac{\lambda}{\lambda_{k+1}}\right)\|v\|_{E}^{2}
$$

Como $u_{t}^{m} \leq 0$, temos

$$
-\frac{1}{2^{*}} \int_{\Omega}\left(v+u_{t}^{m}\right)_{+}^{2^{*}} \geq-\frac{1}{2^{*}} \int_{\Omega}\left|v_{+}\right|^{2^{*}} \geq-\frac{1}{2^{*}} \int_{\Omega}|v|^{2^{*}}=-\frac{1}{2^{*}}\|v\|_{2^{*}}^{2^{*}}
$$

De $\left(g_{2}\right)$ e $\left(g_{3}\right)$ segue que

$$
0 \leq G(x, s) \leq \frac{C_{g}}{p+1} s^{p+1}
$$

com isso temos

$$
-\int_{\Omega} G\left(x,\left(v+u_{t}^{m}\right)_{+}\right) \geq-\frac{C_{g}}{p+1} \int_{\Omega}\left(v+u_{t}^{m}\right)_{+}^{p+1} \geq-\frac{C_{g}}{p+1}\|v\|_{p+1}^{p+1} .
$$

Logo, de (5.22), (5.23), (5.24) e pelo Lema 5.3

$$
I_{m}(v) \geq \frac{1}{2}\left(1-\frac{\lambda}{\lambda_{k+1}}\right)\|v\|_{E}^{2}-\frac{1}{2^{*}}\|v\|_{2^{*}}^{2^{*}}-\frac{C_{g}}{p+1}\|v\|_{p+1}^{p+1}-C_{2} m^{-\frac{N}{2}}\|v\|_{E} .
$$

Pelo item (i) do TEOREMA DE MERGULHO DE SOBOLEV obtemos

$$
I_{m}(v) \geq c_{1}\|v\|_{E}^{2}-c_{2}\|v\|_{E}^{2^{*}}-c_{3}\|v\|_{E}^{p+1}-c_{4} m^{-\frac{N}{2}}\|v\|_{E},
$$

onde $c_{1}, c_{2}, c_{3}, c_{4}>0$ independem de $m$.

Dado $m \in \mathbb{N}$ defina $k_{m}:[0, \infty) \rightarrow \mathbb{R}$ por

$$
k_{m}(s)=c_{1} s^{2}-c_{2} s^{2^{*}}-c_{3} s^{p+1}-c_{4} m^{-\frac{N}{2}} s .
$$

Observando que $2^{*}, p+1>2$, se escolhermos $\rho>0$ de modo que $c_{2} \rho^{2^{*}-2}+c_{3} \rho^{p-1}<c_{1}$, 
podemos escolher em seguida $m_{0}$ suficientemente grande de modo que

$$
c_{4} m_{0}^{-\frac{N}{2}} \rho<c_{1} \rho^{2}-c_{2} \rho^{2^{*}}-c_{3} \rho^{p+1},
$$

o que nos dará $\alpha:=k_{m_{0}}(\rho)>0$. É fácil observar que se $m \geq m_{0}$, então $k_{m_{0}}(s) \leq k_{m}(s)$ para todo $s \in[0, \infty)$; em particular, temos que $\alpha=k_{m_{0}}(\rho) \leq k_{m}(\rho)$. Logo, considerando $\|v\|_{E}=\rho$ em (5.25), obtemos (5.21) para todo $m$ suficientemente grande.

\section{Lema 5.12.}

Se $m \in \mathbb{N}$ for suficientemente grande e $\epsilon>0$ e $\epsilon m$ suficientemente pequenos, então existe $R:=R_{\epsilon, m}>\frac{\rho}{\left\|\Phi_{\epsilon, m}\right\|_{E}}$ tal que

$$
\left.I\right|_{\partial Q_{R, m}^{\epsilon}} \leq 0
$$

\section{Prova.}

Assumiremos que $\epsilon>0$ e $m \in \mathbb{N}$ são tais que $\epsilon<\frac{1}{2 m}$.

Recordemos que o item (a) do Lema 5.5 nos dá $\left\|\Phi_{\epsilon, m}\right\|_{E} \rightarrow S^{N}$ quando $\epsilon m \rightarrow 0$; logo, podemos dizer que existe $\varsigma>0$ tal que para $\epsilon m$ suficientemente pequeno vale

$$
\varsigma<\left\|\Phi_{\epsilon, m}\right\|_{E} .
$$

Sendo assim, estamos agora interessados em verificar se dados $\epsilon>0$ e $m \in \mathbb{N}$ de modo que valha (5.27), existe $R>\frac{\rho}{\varsigma}$ tal que $\left.I\right|_{\partial Q_{R, m}^{\epsilon}} \leq 0$.

Seja $v=w+s \Phi_{\epsilon, m} \in W_{m, k} \oplus[0, \infty) \Phi_{\epsilon, m}$. Devido a (5.18) podemos fazer estimativas para $I_{m}(v)$ estimando separadamente $I_{m}(w)$ e $I_{m}\left(s \Phi_{\epsilon, m}\right)$.

Sabemos que todo $w \in W_{m, k}$ pode ser escrito na forma $w=\zeta_{m} \widetilde{w}$, onde $\widetilde{w} \in W_{k}$. Sendo assim, para todo $w \in W_{m, k}$

$$
\begin{aligned}
\left(F-F_{m}\right)(w) & =\int_{\Omega}\left(\nabla u_{t} \cdot \nabla w-\lambda u_{t} w d x\right)-\int_{\Omega}\left(\nabla u_{t}^{m} \cdot \nabla w-\lambda u_{t}^{m} w d x\right) \\
& =\int_{\Omega} \nabla\left[\left(1-\eta_{m}\right) u_{t}\right] \cdot \nabla(\zeta \widetilde{w}) d x-\lambda \int_{\Omega}\left(1-\eta_{m}\right) \zeta_{m} u_{t} \widetilde{w} d x \\
& =0
\end{aligned}
$$


uma vez que $\operatorname{supp}\left(1-\eta_{m}\right) \subset \bar{B}_{\frac{2}{m}}$ e $\operatorname{supp} \zeta_{m} \subset\left(\Omega \backslash B_{\frac{2}{m}}\right)$. Daí segue que

$$
\begin{aligned}
I_{m}(w)= & \frac{1}{2} \int_{\Omega}|\nabla w|^{2} d x-\frac{\lambda}{2} \int_{\Omega} w^{2} d x-\frac{1}{2^{*}} \int_{\Omega}\left(w+u_{t}^{m}\right)_{+}^{2^{*}} d x-\int_{\Omega} G\left(x,\left(w+u_{t}^{m}\right)_{+}\right) \\
& -\left(F-F_{m}\right)(w) \\
\leq & \frac{1}{2} \int_{\Omega}|\nabla w|^{2} d x-\frac{\lambda}{2} \int_{\Omega} w^{2} d x
\end{aligned}
$$

para todo $w \in W_{m, k}$.

Como supp $\Phi_{\epsilon, m} \subset \bar{B}_{\frac{1}{m}}$ e $\operatorname{supp} u_{t}^{m} \subset \Omega \backslash B_{\frac{1}{m}}$, dado $s \in[0, \infty)$, temos que

$$
\begin{aligned}
I_{m}\left(s \Phi_{\epsilon, m}\right)= & \frac{1}{2} \int_{\Omega}\left|\nabla\left(s \Phi_{\epsilon, m}\right)\right|^{2} d x-\frac{\lambda}{2} \int_{\Omega}\left(s \Phi_{\epsilon, m}\right)^{2} d x-\frac{1}{2^{*}} \int_{\Omega}\left(s \Phi_{\epsilon, m}+u_{t}^{m}\right)_{+}^{2^{*}} d x \\
& -\int_{\Omega} G\left(x,\left(s \Phi_{\epsilon, m}+u_{t}^{m}\right)_{+}\right) d x-\left(F-F_{m}\right)\left(s \Phi_{\epsilon, m}\right) \\
\leq & \frac{s^{2}}{2} \int_{B_{\frac{1}{m}}}\left|\nabla \Phi_{\epsilon, m}\right|^{2} d x-\frac{s^{2^{*}}}{2^{*}} \int_{B_{\frac{1}{m}}} \Phi_{\epsilon, m}^{2^{*}} d x-s \int_{B_{\frac{1}{m}}} f \Phi_{\epsilon, m} d x
\end{aligned}
$$

De (5.28) e (5.29) segue que

$$
\begin{aligned}
I_{m}(v) \leq & \frac{1}{2} \int_{\Omega}|\nabla w|^{2} d x-\frac{\lambda}{2} \int_{\Omega} w^{2} d x+\frac{s^{2}}{2} \int_{B_{\frac{1}{m}}}\left|\nabla \Phi_{\epsilon, m}\right|^{2} d x \\
& -\frac{s^{2^{*}}}{2^{*}} \int_{B_{\frac{1}{m}}} \Phi_{\epsilon, m}^{2^{*}} d x-s \int_{B_{\frac{1}{m}}} f \Phi_{\epsilon, m} d x
\end{aligned}
$$

Escolha $m_{0} \in \mathbb{N}$ tal que $c_{k} m^{-N}<\frac{\lambda-\lambda_{k}}{2}$ para todo $m \geq m_{0}$, onde $c_{k}$ é a constante da estimativa (5.17). De (5.17), (5.28), (5.29), do item (a) do Lema 5.5 e da desigualdade de Hölder segue que

$$
\begin{aligned}
I_{m}(v) \leq & \frac{1}{2}\left(1-\frac{\lambda}{\lambda_{k}+c_{k} m^{-N}}\right)\|w\|_{E}^{2}+\frac{s^{2}}{2} S^{\frac{N}{2}}+D_{1} \frac{s^{2}}{2}(\epsilon m)^{N-2} \\
& -\frac{s^{2^{*}}}{2^{*}} \int_{B_{\frac{1}{m}}} \Phi_{\epsilon, m}^{2^{*}} d x+s\|f\|_{2}\left\|\Phi_{\epsilon, m}\right\|_{2} .
\end{aligned}
$$

Da Observação 5.6, podemos considerar $\epsilon m$ suficientemente pequeno de modo que $\left\|\Phi_{\epsilon, m}\right\|_{2} \leq$ 
1, assim

$$
\begin{aligned}
I_{m}(v) \leq & \frac{1}{2}\left(1-\frac{\lambda}{\lambda_{k}+c_{k} m^{-N}}\right)\|w\|_{E}^{2}+\frac{s^{2}}{2} S^{\frac{N}{2}}+D_{1} \frac{s^{2}}{2}(\epsilon m)^{N-2} \\
& -\frac{s^{2^{*}}}{2^{*}} \int_{B_{\frac{1}{m}}} \Phi_{\epsilon, m}^{2^{*}} d x+s\|f\|_{2} .
\end{aligned}
$$

Como $1-\frac{\lambda}{\lambda_{k}+c_{k} m^{-N}} \underset{m \rightarrow \infty}{\longrightarrow} 1-\frac{\lambda}{\lambda_{k}}<0$, existe $d>0$ tal que $1-\frac{\lambda}{\lambda_{k}+c_{k} m^{-N}}<-2 d$ para todo $m$ suficientemente grande, o que nos dá

$$
I_{m}(v) \leq-d\|w\|_{E}^{2}+\frac{s^{2}}{2} S^{\frac{N}{2}}+D_{1} \frac{s^{2}}{2}(\epsilon m)^{N-2}-\frac{s^{2^{*}}}{2^{*}} \int_{B_{\frac{1}{m}}} \Phi_{\epsilon, m}^{2^{*}} d x+s\|f\|_{2}
$$

para todo $w \in W_{m, k}$ e $s \geq 0$.

Considere $\partial Q_{R, m}^{\epsilon}=\Gamma_{1} \cup \Gamma_{2} \cup \Gamma_{3}$ como em (5.20), ou seja,

$$
\begin{aligned}
& \Gamma_{1}=W_{m, k} \cap \bar{B}_{R}, \\
& \Gamma_{2}=\left\{v \in E: v=w+s \Phi_{\epsilon, m}, w \in W_{m, k},\|w\|_{E}=R \text { e } s \in[0, R]\right\}, \\
& \Gamma_{3}=\left\{v \in E: v=w+R \Phi_{\epsilon, m}, w \in W_{m, k} \cap \bar{B}_{R}\right\} .
\end{aligned}
$$

- Seja $v \in \Gamma_{1}$. Neste caso, basta fazer $s=0$ em (5.30); assim obtemos

$$
I_{m}(v) \leq-d\|w\|_{E}^{2} \leq 0
$$

para qualquer $R$.

- Seja $v \in \Gamma_{3}$, ou seja, $v=w+R \Phi_{\epsilon, m}$, com $\|w\|_{E} \leq R$.

Fazendo $s=R$ em (5.30) e utilizando o item (c) do lema 5.5, temos

$$
\begin{aligned}
I_{m}(v) & \leq \frac{R^{2}}{2} S^{\frac{N}{2}}+D_{1} \frac{R^{2}}{2}(\epsilon m)^{N-2}-\frac{R^{2^{*}}}{2^{*}} K_{1}+R\|f\|_{2} \\
& =\left(\frac{S^{\frac{N}{2}}}{2}+\frac{D_{1}}{2}(\epsilon m)^{N-2}\right) R^{2}-\frac{R^{2^{*}}}{2^{*}} K_{1}+R\|f\|_{2} .
\end{aligned}
$$


Como $\epsilon m<1$, temos

$$
I_{m}(v) \leq\left(\frac{S^{\frac{N}{2}}}{2}+\frac{D_{1}}{2}\right) R^{2}-\frac{K_{1}}{2^{*}} R^{2^{*}}+R\|f\|_{2} .
$$

Fazendo $d_{1}=\left(\frac{S^{\frac{N}{2}}}{2}+\frac{D_{1}}{2}\right), d_{2}=\frac{K_{1}}{2^{*}}$ e $d_{3}=\|f\|_{2}$, podemos escrever

$$
I_{m}(v) \leq d_{1} R^{2}-d_{2} R^{2^{*}}+d_{3} R
$$

Logo, existe $R_{1}>0$ tal que para todo $R \geq R_{1}$ vale dizer que $I_{m}(v) \leq 0$.

- Seja $v \in \Gamma_{2}$, ou seja, $v=w+s \Phi_{\epsilon, m}$, com $\|w\|_{E}=R$ e $s \in[0, R]$.

De (5.30) e do item (b) do Lema 5.5, obtemos

$$
\begin{aligned}
I_{m}(v) & \leq-d\|w\|_{E}^{2}+\frac{s^{2}}{2} S^{\frac{N}{2}}+D_{1} \frac{s^{2}}{2}(\epsilon m)^{N-2}-\frac{s^{2^{*}}}{2^{*}} S^{\frac{N}{2}}+D_{2} \frac{s^{2^{*}}}{2^{*}}(\epsilon m)^{N}+s\|f\|_{2} \\
& =-d\|w\|_{E}^{2}+\left(\frac{s^{2}}{2}-\frac{s^{2^{*}}}{2^{*}}\right) S^{\frac{N}{2}}+D_{1} \frac{s^{2}}{2}(\epsilon m)^{N-2}+D_{2} \frac{s^{2^{*}}}{2^{*}}(\epsilon m)^{N}+s\|f\|_{2}
\end{aligned}
$$

Como $\frac{s^{2}}{2}-\frac{s^{2^{*}}}{2^{*}}$ atinge seu máximo $1 / N$ quando $s=1$, então

$$
I_{m}(v) \leq-d\|w\|_{E}^{2}+\frac{1}{N} S^{\frac{N}{2}}+D_{1} \frac{s^{2}}{2}(\epsilon m)^{N-2}+D_{2} \frac{s^{2^{*}}}{2^{*}}(\epsilon m)^{N}+s\|f\|_{2} .
$$

Por fim, como $\|w\|_{E}=R$ e $s \leq R$, obtemos

$$
\begin{aligned}
I_{m}(v) & \leq-d R^{2}+\frac{1}{N} S^{\frac{N}{2}}+D_{1} \frac{R^{2}}{2}(\epsilon m)^{N-2}+D_{2} \frac{R^{2^{*}}}{2^{*}}(\epsilon m)^{N}+R\|f\|_{2} \\
& =-d R^{2}+\frac{1}{N} S^{\frac{N}{2}}+\left(\frac{D_{1}}{2} R^{2-2^{*}}+\frac{D_{2}}{2^{*}}(\epsilon m)^{2}\right) R^{2^{*}}(\epsilon m)^{N-2}+R\|f\|_{2} .
\end{aligned}
$$

Assumindo que $R>1$ e lembrando que $\epsilon m<1$ podemos considerar $d_{0}=\frac{1}{N} S^{\frac{N}{2}}$, $d_{1}=2 \max \left\{\frac{D_{1}}{2}, \frac{D_{2}}{2^{*}}\right\}$ e $d_{2}=\|f\|_{2}$ de modo a obtermos

$$
I_{m}(v) \leq-d R^{2}+d_{0}+d_{1} R^{2^{*}}(\epsilon m)^{N-2}+d_{2} R
$$


Escolha $\tau \in\left(\frac{(N-2)^{2}}{2 N}, \frac{(N-2)^{2}}{4}\right)$ e considere $R:=R_{\epsilon, m}=(\epsilon m)^{-\tau}$. Desse modo obtemos

$$
I_{m}(v) \leq-d R^{2}+d_{0}+d_{1} R^{2^{*}-\frac{N-2}{\tau}}+d_{2} R
$$

Como $0<2^{*}-\frac{N-2}{\tau}<2$, podemos dizer que existem $\epsilon_{0}>0$ e $m_{0} \in \mathbb{N}$, com $\epsilon_{0} m_{0}<$ 1 , tais que se $\epsilon m \leq \epsilon_{0} m_{0}$, então quando considerarmos $R_{\epsilon, m}$ em (5.32), obteremos $I_{m}(v) \leq 0$.

Considere $R_{0}=R_{\epsilon_{0}, m_{0}}$ e faça $\bar{R}=\max \left\{R_{0}, R_{1}, \frac{\rho}{\varsigma}, \rho\right\}$. De acordo com o que vimos até aqui nesta demonstração, podemos dizer que se $\epsilon$ e $m$ forem escolhidos de modo que valham (5.27) e $R=R_{\epsilon, m}>\bar{R}$, então teremos $I_{m}(v) \leq 0$ para todo $v \in \partial Q_{R, m}^{\epsilon}$.

A partir daqui, consideraremos $\epsilon_{m}=m^{-\frac{1}{\beta}}$, onde, por enquanto, assumiremos $\beta \in(0,1)$. Desta maneira, $\epsilon_{m} \rightarrow 0$ e $\epsilon_{m} m=m^{1-\frac{1}{\beta}} \rightarrow 0$ quando $m \rightarrow \infty$. Assim, se $m \in \mathbb{N}$ for suficientemente grande, $I_{m}$ satisfaz as hipóteses do TEOREMA DE ENLACE; logo podemos dizer que em um nível $c_{m}$ existe uma sequência $(P S)$ para $I_{m}$ que denotaremos por $\left\{v_{n}\right\}_{n \in \mathbb{N}}$. Como dito no início desta seção, a sequência $\left\{v_{n}\right\}_{n \in \mathbb{N}}$ depende de $m$, no entanto, tal dependência será omitida a fim de não sobrecarregar a notação.

Segundo o TEOREMA DE ENLACE podemos concluir que

$$
I_{m}\left(v_{n}\right) \underset{n \rightarrow \infty}{\longrightarrow} c_{m} \quad \text { e } \quad I_{m}^{\prime}\left(v_{n}\right) \underset{n \rightarrow \infty}{\longrightarrow} 0
$$

onde

$$
c_{m}=\inf _{h \in \Gamma} \max _{u \in Q_{R, m}^{\epsilon_{m}}} I_{m}(h(u)) \geq \alpha
$$

$\alpha$ vem do Lema 5.11 e $\Gamma=\left\{h \in C\left(Q_{R, m}^{\epsilon_{m}}, E\right): \quad h(u)=u \quad\right.$ se $\left.\quad u \in \partial Q_{R, m}^{\epsilon_{m}}\right\}$, lembrando que $R$ é escolhido oportunamente em função de $m$, de acordo com o Lema 5.12.

Sem perda de generalidade, suporemos que existe uma sequência $\left\{\delta_{n}\right\} \subset(0,1)$, com $\delta_{n} \underset{n \rightarrow \infty}{\longrightarrow} 0$, tal que

$$
\begin{aligned}
& \left|I_{m}\left(v_{n}\right)-c_{m}\right| \leq \delta_{n} \\
& \left|I_{m}^{\prime}\left(v_{n}\right) \cdot \varphi\right| \leq \delta_{n}|| \varphi \|_{E} \quad \forall \varphi \in E .
\end{aligned}
$$




\subsection{A solução fraca}

Nesta seção mostraremos que a sequência $\left\{v_{n}\right\}_{n \in \mathbb{N}}$, obtida na seção 5.7, é limitada em $E$. Uma vez verificado esse fato, poderemos dizer que existe $v_{0} \in E$ tal que $v_{n} \rightarrow_{n \rightarrow \infty} v_{0}$. Devemos sempre recordar que $\left\{v_{n}\right\}_{n \in \mathbb{N}}$ depende de $m$, consequentemente seu limite fraco também dependerá, mas não explicitaremos isso a fim de não carregar a notação.

Será verificado ainda nesta seção que o limite fraco $v_{0}$ é solução fraca de $\left(P_{m}\right)$.

\section{Lema 5.13.}

Sob as hipóteses dos Teoremas 5.1 ou 5.2, a sequência $\left\{v_{n}\right\}_{n \in \mathbb{N}}$ é limitada em E.

\section{Prova.}

Observe que

$$
\begin{aligned}
I_{m}\left(v_{n}\right)-\frac{1}{2} I_{m}^{\prime}\left(v_{n}\right) \cdot v_{n}= & \frac{1}{N} \int_{\Omega}\left(v_{n}+u_{t}^{m}\right)_{+}^{2^{*}} d x-\frac{1}{2} \int_{\Omega}\left(v_{n}+u_{t}^{m}\right)_{+}^{2^{*}-1} u_{t}^{m} d x \\
& -\int_{\Omega} G\left(x,\left(v_{n}+u_{t}^{m}\right)_{+}\right) d x+\frac{1}{2} \int_{\Omega} g\left(x,\left(v_{n}+u_{t}^{m}\right)_{+}\right) v_{n} d x \\
& -\frac{1}{2}\left(F-F_{m}\right)\left(v_{n}\right) .
\end{aligned}
$$

Como $u_{t}^{m} \leq 0$ e $g\left(x,\left(v_{n}+u_{t}^{m}\right)\right) v_{n} \geq 0$, podemos descartar o segundo e o quarto termo a direita, obtendo

$$
\frac{1}{N} \int_{\Omega}\left(v_{n}+u_{t}^{m}\right)_{+}^{2^{*}} d x \leq I_{m}\left(v_{n}\right)-\frac{1}{2} I_{m}^{\prime}\left(v_{n}\right) \cdot v_{n}+\int_{\Omega} G\left(x,\left(v_{n}+u_{t}^{m}\right)_{+}\right) d x+\frac{1}{2}\left(F-F_{m}\right)\left(v_{n}\right) .
$$

Do Lema 5.3, de (5.34) e de (5.35), obtemos

$$
\frac{1}{N} \int_{\Omega}\left(v_{n}+u_{t}^{m}\right)_{+}^{2^{*}} d x \leq \int_{\Omega} G\left(x,\left(v_{n}+u_{t}^{m}\right)_{+}\right) d x+c_{m}+\delta_{n}+\frac{\delta_{n}}{2}\left\|v_{n}\right\|_{E}+\frac{1}{2} C_{2}(N) m^{-\frac{N}{2}}\left\|v_{n}\right\|_{E}
$$

Usando a condição $\left(g_{3}\right)$ segue que

$$
\frac{1}{N} \int_{\Omega}\left(v_{n}+u_{t}^{m}\right)_{+}^{2^{*}} d x \leq \frac{C_{g}}{p+1} \int_{\Omega}\left(v_{n}+u_{t}^{m}\right)_{+}^{p+1} d x+c_{1}+c_{2}\left\|v_{n}\right\|_{E}
$$

Pelo TEOREMA DE MERGUlho DE SOBOLEV $\left(v_{n}+u_{t}^{m}\right)_{+} \in L^{2^{*}}(\Omega)$. Como $p+1<2^{*}$, 
obtemos

$$
\frac{1}{N} \int_{\Omega}\left(v_{n}+u_{t}^{m}\right)_{+}^{2^{*}} d x \leq c_{0}\left(\int_{\Omega}\left(v_{n}+u_{t}^{m}\right)_{+}^{2^{*}} d x\right)^{\frac{p+1}{2^{*}}}+c_{1}+c_{2}\left\|v_{n}\right\|_{E}
$$

equivalentemente

$$
\int_{\Omega}\left(v_{n}+u_{t}^{m}\right)_{+}^{2^{*}} d x-c_{0}\left(\int_{\Omega}\left(v_{n}+u_{t}^{m}\right)_{+}^{2^{*}} d x\right)^{\frac{p+1}{2^{*}}} \leq c_{1}+c_{2}\left\|v_{n}\right\|_{E}
$$

Seja $h:(0, \infty) \rightarrow \mathbb{R}$ dada por

$$
h(x)=1-c x^{\frac{p+1}{2^{*}-1}} .
$$

Como $h$ é contínua e $h(x) \underset{x \rightarrow \infty}{\longrightarrow} 1$, existe $\bar{x} \in(0, \infty)$ tal que para todo $x \geq \bar{x}$, teremos $\frac{1}{2} \leq h(x)$. Observe que $\bar{x}$ não depende de $n$ nem de $m$.

Se $\int_{\Omega}\left(v_{n}+u_{t}^{m}\right)_{+}^{2^{*}} d x \geq \bar{x}$, então

$$
\frac{1}{2} \leq 1-c\left(\int_{\Omega}\left(v_{n}+u_{t}^{m}\right)_{+}^{2^{*}} d x\right)^{\frac{p+1}{2^{*}}-1}
$$

o que nos dá

$$
\begin{aligned}
\frac{1}{2} \int_{\Omega}\left(v_{n}+u_{t}^{m}\right)_{+}^{2^{*}} d x & \leq \int_{\Omega}\left(v_{n}+u_{t}^{m}\right)_{+}^{2^{*}} d x-c\left(\int_{\Omega}\left(v_{n}+u_{t}^{m}\right)_{+}^{2^{*}} d x\right)^{\frac{p+1}{2^{*}}} \\
& \leq c_{1}+c_{2}\left\|v_{n}\right\|_{E}
\end{aligned}
$$

Se $\int_{\Omega}\left(v_{n}+u_{t}^{m}\right)_{+}^{2^{*}} d x \leq \bar{x}$, então

$$
\begin{aligned}
\frac{1}{2} \int_{\Omega}\left(v_{n}+u_{t}^{m}\right)_{+}^{2^{*}} d x & \leq \frac{1}{2} \int_{\Omega}\left(v_{n}+u_{t}^{m}\right)_{+}^{2^{*}} d x+c_{1}+c_{2}\left\|v_{n}\right\|_{E} \\
& \leq \frac{1}{2} \bar{x}+c_{1}+c_{2}\left\|v_{n}\right\|_{E}
\end{aligned}
$$

Logo, existem novas constantes positivas $c_{1}$ e $c_{2}$ tais que

$$
\left\|\left(v_{n}+u_{t}^{m}\right)_{+}\right\|_{2^{*}}^{2^{*}}=\int_{\Omega}\left(v_{n}+u_{t}^{m}\right)_{+}^{2^{*}} d x \leq c_{1}+c_{2}\left\|v_{n}\right\|_{E} \quad \forall n \in \mathbb{N} .
$$

Considere $v_{n}=w_{n}+z_{n}$ onde $w_{n} \in W_{k}=\left\langle\phi_{i}\right\rangle_{i=1}^{k}$ e $z_{n} \in X_{k}=\left(W_{k}\right)^{\perp}$. 
Usando o item (b) da Proposição A.1 e em seguida a estimativa (5.35), temos

$$
\begin{aligned}
\left(1-\frac{\lambda}{\lambda_{k+1}}\right)\left\|z_{n}\right\|_{E}^{2} \leq & \int_{\Omega}\left|\nabla z_{n}\right|^{2} d x-\lambda \int_{\Omega}\left|z_{n}\right|^{2} d x \\
= & \int_{\Omega} \nabla v_{n} \cdot \nabla z_{n} d x-\lambda \int_{\Omega} v_{n} z_{n} d x \\
\leq & \int_{\Omega}\left(v_{n}+u_{t}^{m}\right)_{+}^{2^{*}-1}\left|z_{n}\right| d x+\int_{\Omega} g\left(x,\left(v_{n}+u_{t}^{m}\right)_{+}\right)\left|z_{n}\right| d x \\
& +\left(F-F_{m}\right)\left(z_{n}\right)+\delta_{n}\left\|z_{n}\right\|_{E} .
\end{aligned}
$$

Usando, agora, a condição $\left(g_{3}\right)$ e o Lema 5.3 obtemos

$$
\begin{aligned}
\left(1-\frac{\lambda}{\lambda_{k+1}}\right)\left\|z_{n}\right\|_{E}^{2} \leq & \int_{\Omega}\left(v_{n}+u_{t}^{m}\right)_{+}^{2^{*}-1}\left|z_{n}\right| d x+C_{g} \int_{\Omega}\left(v_{n}+u_{t}^{m}\right)_{+}^{p}\left|z_{n}\right| d x \\
& +C_{2}(N) m^{-\frac{N}{2}}|| z_{n}\left\|_{E}+\delta_{n}|| z_{n}\right\|_{E} \\
\leq & \int_{\Omega}\left(v_{n}+u_{t}^{m}\right)_{+}^{2^{*}-1}\left|z_{n}\right| d x+C_{g} \int_{\Omega}\left(v_{n}+u_{t}^{m}\right)_{+}^{p}\left|z_{n}\right| d x+k_{1}\left\|z_{n}\right\|_{E} .
\end{aligned}
$$

Da desigualdade de Hölder

$$
\begin{aligned}
\left(1-\frac{\lambda}{\lambda_{k+1}}\right)\left\|z_{n}\right\|_{E}^{2} \leq & \left\|\left(v_{n}+u_{t}^{m}\right)_{+}\right\|_{2^{*}}^{2^{*}-1}\left\|z_{n}\right\|_{2^{*}} \\
& +C_{g}\left\|\left(v_{n}+u_{t}^{m}\right)_{+}\right\| 2_{2^{*}}^{p}\left\|z_{n}\right\|_{\frac{2^{*}}{2^{*}-p}}+k_{1}\left\|z_{n}\right\|_{E} .
\end{aligned}
$$

Agora, usaremos a desigualdade de Young com $\epsilon>0$ dado para obtermos

$$
\begin{aligned}
\left(1-\frac{\lambda}{\lambda_{k+1}}\right)\left\|z_{n}\right\|_{E}^{2} \leq & \left.\frac{1}{\epsilon} \|\left(v_{n}+u_{t}^{m}\right)_{+}\right)\left\|_{2^{*}}^{2\left(2^{*}-1\right)}+\epsilon\right\| z_{n} \|_{2^{*}}^{2} \\
& +\frac{C_{g}}{\epsilon}\left\|\left(v_{n}+u_{t}^{m}\right)_{+}\right\|_{2^{*}}^{2 p}+C_{g} \epsilon\left\|z_{n}\right\|_{2_{2^{*}-p}}^{2}+k_{1}\left\|z_{n}\right\|_{E} \\
= & \left.\frac{1}{\epsilon}\left(\|\left(v_{n}+u_{t}^{m}\right)_{+}\right) \|_{2^{*}}^{2^{*}}\right)^{\frac{2\left(2^{*}-1\right)}{2^{*}}}+\epsilon\left\|z_{n}\right\|_{2^{*}}^{2} \\
& +\frac{C_{g}}{\epsilon}\left(\left\|\left(v_{n}+u_{t}^{m}\right)_{+}\right\| 2_{2^{*}}^{2^{*}}\right)^{\frac{2 p}{2^{*}}}+C_{g} \epsilon\left\|z_{n}\right\|_{\frac{2^{*}}{2^{*}-p}}^{2}+k_{1}\left\|z_{n}\right\|_{E} .
\end{aligned}
$$


De (5.36)

$$
\begin{aligned}
\left(1-\frac{\lambda}{\lambda_{k+1}}\right)\left\|z_{n}\right\|_{E}^{2} \leq & \frac{1}{\epsilon}\left(c_{1}+c_{2}\left\|v_{n}\right\|_{E}\right)^{\frac{N+2}{N}}+\frac{C_{g}}{\epsilon}\left(c_{1}+c_{2}\left\|v_{n}\right\|_{E}\right)^{\frac{2 p}{2^{*}}} \\
& +\epsilon\left\|z_{n}\right\|_{2^{*}}^{2}+C_{g} \epsilon\left\|z_{n}\right\|_{\frac{2^{*}}{2^{*}-p}}^{2}+k_{1}\left\|z_{n}\right\|_{E} \\
\leq & \frac{1}{\epsilon}\left[2^{\frac{N+2}{2}}\left(c_{1}^{\frac{N+2}{2}}+c_{2}^{\frac{N+2}{2}}\left\|v_{n}\right\|_{E}^{\frac{N+2}{2}}\right)\right]+\frac{C_{g}}{\epsilon}\left[2^{\frac{2 p}{2^{*}}}\left(c_{1}^{\frac{2 p}{22^{*}}}+c_{2}^{\frac{2 p}{2^{*}}}\left\|v_{n}\right\|_{E}^{\frac{2 p}{2^{*}}}\right)\right] \\
& +\epsilon\left\|z_{n}\right\|_{2^{*}}^{2}+C_{g} \epsilon\left\|z_{n}\right\|_{2_{2^{*}}}^{2}+k_{1}\left\|z_{n}\right\|_{E} \\
= & \frac{1}{\epsilon} d_{5}+\frac{1}{\epsilon}\left(d_{4}\left\|v_{n}\right\|_{E}^{\frac{N+2}{N}}+d_{3}\left\|v_{n}\right\|_{E}^{\frac{2 p}{2^{*}}}\right) \\
& +\epsilon\left\|z_{n}\right\|_{2^{*}}^{2}+C_{g} \epsilon\left\|z_{n}\right\|_{\frac{2^{*}}{2^{*}-p}}^{2}+k_{1}\left\|z_{n}\right\|_{E} .
\end{aligned}
$$

Agora pelo TEOREMA DE MERGULHO DE SOBOLEV

$$
\begin{aligned}
\left(1-\frac{\lambda}{\lambda_{k+1}}\right)\left\|z_{n}\right\|_{E}^{2} \leq & \frac{1}{\epsilon} d_{5}+\frac{1}{\epsilon}\left(d_{4}\left\|v_{n}\right\|_{E}^{\frac{N+2}{N}}+d_{3}\left\|v_{n}\right\|_{E}^{\frac{2 p}{2^{*}}}\right) \\
& +\epsilon\left\|z_{n}\right\|_{E}^{2}+C_{g} \epsilon\left\|z_{n}\right\|_{E}^{2}+k_{1}\left\|z_{n}\right\|_{E} \\
= & \frac{1}{\epsilon} d_{5}+\frac{1}{\epsilon}\left(d_{4}\left\|v_{n}\right\|_{E}^{\frac{N+2}{N}}+d_{3}\left\|v_{n}\right\|_{E}^{\frac{2 p}{2^{*}}}\right) \\
& +\epsilon d_{2}\left\|z_{n}\right\|_{E}^{2}+k_{1}\left\|z_{n}\right\|_{E},
\end{aligned}
$$

equivalentemente

$$
\left(1-\frac{\lambda}{\lambda_{k+1}}-\epsilon d_{2}\right)\left\|z_{n}\right\|_{E}^{2} \leq \frac{1}{\epsilon} d_{5}+\frac{1}{\epsilon}\left(d_{4}\left\|v_{n}\right\|_{E}^{\frac{N+2}{N}}+d_{3}\left\|v_{n}\right\|_{E}^{\frac{2 p}{2^{*}}}\right)+k_{1}\left\|z_{n}\right\|_{E} .
$$

Fixando $\epsilon_{0} \in\left(0, \frac{1}{d_{2}}\left(1-\frac{\lambda}{\lambda_{k+1}}\right)\right)$ e considerando $k_{0}=1-\frac{\lambda}{\lambda_{k+1}}-\epsilon_{0} d_{2}$, obtemos finalmente

$$
\begin{aligned}
k_{0}\left\|z_{n}\right\|_{E}^{2} & \leq k_{4}+k_{3}\left\|v_{n}\right\|_{E}^{\frac{N+2}{N}}+k_{2}\left\|v_{n}\right\|_{E}^{\frac{2 p}{2^{*}}}+k_{1}\left\|z_{n}\right\|_{E} \\
& \leq k_{4}+k_{3}\left\|v_{n}\right\|_{E}^{\frac{N+2}{N}}+k_{2}\left\|v_{n}\right\|_{E}^{\frac{2 p}{2^{*}}}+k_{1}\left\|v_{n}\right\|_{E} .
\end{aligned}
$$

Procuramos agora uma estimativa análoga para a componente $w_{n}$. Usando o item $(a)$ 
da Proposição A.1 e em seguida a estimativa (5.35), temos

$$
\begin{aligned}
\left(\frac{\lambda}{\lambda_{k}}-1\right) \|\left. w_{n}\right|_{E} ^{2} \leq & \lambda \int_{\Omega}\left|w_{n}\right|^{2} d x-\int_{\Omega}\left|\nabla w_{n}\right|^{2} d x \\
= & \lambda \int_{\Omega} v_{n} w_{n} d x-\int_{\Omega} \nabla v_{n} \cdot \nabla w_{n} d x \\
\leq & \int_{\Omega}\left(v_{n}+u_{t}^{m}\right)_{+}^{2^{*}-1}\left|w_{n}\right| d x+\int_{\Omega} g\left(x,\left(v_{n}+u_{t}^{m}\right)_{+}\right)\left|w_{n}\right| d x \\
& +\left(F-F_{m}\right)\left(w_{n}\right)+\delta_{n}\left\|w_{n}\right\|_{E} .
\end{aligned}
$$

Usando a condição $\left(g_{3}\right)$ e o Lema 5.3 obtemos

$$
\begin{aligned}
\left(\frac{\lambda}{\lambda_{k}}-1\right)\left\|w_{n}\right\|_{E}^{2} \leq & \int_{\Omega}\left(v_{n}+u_{t}^{m}\right)_{+}^{2^{*}-1}\left|w_{n}\right| d x+C_{g} \int_{\Omega}\left(v_{n}+u_{t}^{m}\right)_{+}^{p}\left|w_{n}\right| d x \\
& +c m^{-\frac{N}{2}}|| w_{n}\left\|_{E}+\delta_{n}|| w_{n}\right\|_{E} \\
\leq & \int_{\Omega}\left(v_{n}+u_{t}^{m}\right)_{+}^{2^{*}-1}\left|w_{n}\right| d x+C_{g} \int_{\Omega}\left(v_{n}+u_{t}^{m}\right)_{+}^{p}\left|w_{n}\right| d x+k_{1}|| w_{n} \|_{E} .
\end{aligned}
$$

A partir daqui, procedemos de modo idêntico ao feito para o caso $z_{n}$ e chegamos em

$$
\begin{aligned}
b_{0}\left\|w_{n}\right\|_{E}^{2} & \leq b_{4}+b_{3}\left\|v_{n}\right\|_{E}^{\frac{N+2}{N}}+b_{2}\left\|v_{n}\right\|_{E}^{\frac{2 p}{2^{*}}}+b_{1}\left\|w_{n}\right\|_{E} \\
& \leq b_{4}+b_{3}\left\|v_{n}\right\|_{E}^{\frac{N+2}{N}}+b_{2}\left\|v_{n}\right\|_{E}^{\frac{2 p}{2^{*}}}+b_{1}\left\|v_{n}\right\|_{E} .
\end{aligned}
$$

De 5.37 e de 5.38 segue

$$
\left\|v_{n}\right\|_{E}^{2}=\left\|w_{n}\right\|_{E}^{2}+\left\|z_{n}\right\|_{E}^{2} \leq a_{4}+a_{3}\left\|v_{n}\right\|_{E}^{\frac{N+2}{N}}+a_{2}\left\|v_{n}\right\|_{E}^{\frac{2 p}{2 *}}+a_{1}\left\|v_{n}\right\|_{E}
$$

Observando que $\frac{N+2}{N}, \frac{2 p}{2^{*}}<2$, podemos concluir que $\left\|v_{n}\right\|_{E}$ é uma sequência limitada.

Como $\left\{v_{n}\right\}_{n \in \mathbb{N}}$ é limitada, existe $v_{0} \in E$ tal que, passando para uma subsequência se necessário, vale

$$
\begin{array}{ll}
v_{n} \rightarrow_{n \rightarrow \infty} v_{0} & \text { em } E \\
v_{n} \underset{n \rightarrow \infty}{\longrightarrow} v_{0} & \text { em } L^{q}(\Omega) \operatorname{com} q \in\left[1,2^{*}\right) \\
v_{n} \underset{n \rightarrow \infty}{\longrightarrow} v_{0} & \text { q.t.p. em } \Omega
\end{array}
$$




\section{Proposição 5.14.}

O limite fraco vo é uma solução fraca do problema $\left(P_{m}\right)$.

\section{Prova.}

Dado $\varphi \in E$, observe que

$$
\left|I_{m}^{\prime}\left(v_{0}\right) \cdot \varphi\right| \leq\left|I_{m}^{\prime}\left(v_{n}\right) \cdot \varphi\right|+\left|I_{m}^{\prime}\left(v_{n}\right) \cdot \varphi-I_{m}^{\prime}\left(v_{0}\right) \cdot \varphi\right|
$$

Por (5.35), $\left|I_{m}^{\prime}\left(v_{n}\right) \cdot \varphi\right| \underset{n \rightarrow \infty}{\longrightarrow} 0$. Se mostrarmos que $\left|I_{m}^{\prime}\left(v_{n}\right) \cdot \varphi-I_{m}^{\prime}\left(v_{0}\right) \cdot \varphi\right| \underset{n \rightarrow \infty}{\longrightarrow} 0$, então poderemos concluir que

$$
\begin{aligned}
\int_{\Omega} \nabla v_{0} \cdot \nabla \varphi d x-\lambda \int_{\Omega} v_{0} \varphi d x & -\int_{\Omega}\left(v_{0}+u_{t}^{m}\right)_{+}^{2^{*}-1} \varphi d x \\
& -\int_{\Omega} g\left(x,\left(v_{0}+u_{t}^{m}\right)_{+}\right) \varphi d x-\left(F-F_{m}\right)(\varphi)=0
\end{aligned}
$$

para todo $\varphi \in E$, o que provará a Proposição.

Observe que

$$
\begin{aligned}
\left|I_{m}^{\prime}\left(v_{n}\right) \cdot \varphi-I_{m}^{\prime}\left(v_{0}\right) \cdot \varphi\right| \leq & \left|\int_{\Omega} \nabla v_{n} \cdot \nabla \varphi d x-\int_{\Omega} \nabla v_{0} \cdot \nabla \varphi d x\right| \\
& +\left|\int_{\Omega} v_{n} \varphi d x-\int_{\Omega} v_{0} \varphi d x\right| \\
& +\left|\int_{\Omega}\left(v_{n}+u_{t}^{m}\right)_{+}^{2^{*}-1} \varphi d x-\int_{\Omega}\left(v_{0}+u_{t}^{m}\right)_{+}^{2^{*}-1} \varphi d x\right| \\
& +\left|\int_{\Omega} g\left(x,\left(v_{n}+u_{t}^{m}\right)_{+}\right) \varphi d x-\int_{\Omega} g\left(x,\left(v_{0}+u_{t}^{m}\right)_{+}\right) \varphi d x\right| .
\end{aligned}
$$

O primeiro termo do lado direito da desigualdade acima tende a 0 por (5.39); o segundo tende a 0 por $(5.40) \operatorname{com} q=2$.

Considere $h_{n}=\left(v_{n}+u_{t}^{m}\right)_{+}^{2^{*}-1}$ e $h_{0}=\left(v_{0}+u_{t}^{m}\right)_{+}^{2^{*}-1}$. Note que $h_{n} \underset{n \rightarrow \infty}{\longrightarrow} h_{0}$ q.t.p. e que

$$
\int_{\Omega}\left|h_{n}\right|^{\frac{2^{*}}{2^{*}-1}} d x=\int_{\Omega}\left(v_{n}+u_{t}^{m}\right)_{+}^{2^{*}} d x \leq\left\|v_{n}+u_{t}^{m}\right\|_{2^{*}}^{2^{*}} \leq C\left\|v_{n}+u_{t}^{m}\right\|_{E}^{2^{*}} \quad \forall n \geq 0 .
$$

Como $v_{n}+u_{t}^{m}$ é uma sequência limitada em $E$, segue que $h_{n}$ é limitada em $L^{\frac{2^{*}}{2^{*}-1}}(\Omega)$, $\operatorname{logo} h_{n} \rightarrow_{n \rightarrow \infty} h_{0}$ em $L^{\frac{2^{*}}{2^{*}-1}}(\Omega)$. Como $\varphi \in E \hookrightarrow L^{2^{*}}(\Omega)$ e como $\frac{2^{*}}{2^{*}-1}$ e $2^{*}$ são expoente 
conjugados, obtemos

$$
\left|\int_{\Omega}\left(v_{n}+u_{t}^{m}\right)_{+}^{2^{*}-1} \varphi d x-\int_{\Omega}\left(v_{0}+u_{t}^{m}\right)_{+}^{2^{*}-1} \varphi d x\right| \underset{n \rightarrow \infty}{\longrightarrow} 0
$$

Como $g$ é contínua, está bem definido o operador de Nemytskii associado dado por $N_{g}(u)=g(\cdot, u)$. Além disso, usando o Lema B.3 com a condição $\left(g_{3}\right)$ podemos dizer que $N_{g}: L^{\frac{2^{*} p}{2^{*}-1}}(\Omega) \rightarrow L^{\frac{2^{*}}{2^{*}-1}}(\Omega)$ é contínuo. Como $1<\frac{2^{*} p}{2^{*}-1}<2^{*}$, de $(5.40)$ segue que $v_{n} \underset{n \rightarrow \infty}{\longrightarrow} v_{0}$ em $L^{\frac{2^{*} p}{2^{*}-1}}(\Omega)$, consequentemente $\left(v_{n}+u_{t}^{m}\right)_{+} \underset{n \rightarrow \infty}{\longrightarrow}\left(v_{0}+u_{t}^{m}\right)_{+}$em $L^{\frac{2^{*} p}{2^{*}-1}}(\Omega) ; \log 0$

$$
\left\|N_{g}\left(\left(v_{n}+u_{t}^{m}\right)_{+}\right)-N_{g}\left(\left(v_{0}+u_{t}^{m}\right)_{+}\right)\right\|_{\frac{2^{*}}{2^{*}-1}} \underset{n \rightarrow \infty}{\longrightarrow} 0 .
$$

Como $\varphi \in E \hookrightarrow L^{2^{*}}(\Omega)$ e como $\frac{2^{*}}{2^{*}-1}$ e $2^{*}$ são expoente conjugados, obtemos

$$
\left|\int_{\Omega} g\left(x,\left(v_{n}+u_{t}^{m}\right)_{+}\right) \varphi d x-\int_{\Omega} g\left(x,\left(v_{0}+u_{t}^{m}\right)_{+}\right) \varphi d x\right| \underset{n \rightarrow \infty}{\longrightarrow} 0 .
$$

Temos, então, que $\left|I_{m}^{\prime}\left(v_{n}\right) \cdot \varphi-I_{m}^{\prime}\left(v_{0}\right) \cdot \varphi\right| \underset{n \rightarrow \infty}{\longrightarrow} 0$ e isso prova a Proposição.

Nosso trabalho estaria encerrado por aqui se não fosse a possibilidade de $v_{0} \equiv u_{t}-u_{t}^{m}$, que satisfaz trivialmente o problema $\left(P_{m}\right)$. Nosso objetivo agora é mostrar que para $m \in \mathbb{N}$ suficientemente grande teremos $v_{0} \not \equiv u_{t}-u_{t}^{m}$. Estamos, então, interessados em comparar o nível crítico $I_{m}\left(v_{0}\right)$ com o nível associado à função $u_{t}-u_{t}^{m}$ para cada $m$. Se nessa comparação pudermos mostrar que os níveis correspondentes são distintos, então poderemos concluir que $v_{0} \not \equiv u_{t}-u_{t}^{m}$. Precisamos, então, de estimativas para os níveis críticos $I_{m}\left(v_{0}\right)$.

\subsection{Estimando os níveis de minimax}

Nesta seção estimaremos os níveis de minimax $c_{m}$ nos seguintes lemas:

\section{Lema 5.15.}

Seja $\mu>0$ tal que $\frac{1}{\mu}+\frac{1}{r}+\frac{1}{2}=1$. Sob as hipóteses do Teorema 5.1 (em particular, com $N \geq 6$ ), é possivel fixar $L>0$ tal que para qualquer $\beta \in\left(\frac{\mu}{N}, \frac{N-4}{N-2}\right) \neq \emptyset$ e m suficientemente grande, vale

$$
c_{m} \leq \frac{1}{N} S^{\frac{N}{2}}-L m^{-\frac{2}{\beta}}
$$




\section{Lema 5.16.}

Sejam $\mu>0$ tal que $\frac{1}{\mu}+\frac{1}{r}+\frac{1}{2}=1$. Sob as hipóteses do Teorema 5.2 (em particular, $N=3,4,5)$, podemos fixar q tal que

$$
2^{*}>q+1> \begin{cases}\frac{2}{3} 2^{*} & \text { se } N=4,5 \\ \frac{9}{2} & \text { se } N=3\end{cases}
$$

e $L>0$, tais que, para todo $\beta$ satisfazendo

$$
\frac{1}{2}(q+1)-\frac{2}{N-2}>\beta> \begin{cases}\frac{2(N-1)}{N-2}-(q+1) & \text { se } N=4,5 \\ \frac{5-(q+1)}{2} & \text { se } N=3\end{cases}
$$

e $m$ suficientemente grande, vale

$$
c_{m} \leq \frac{1}{N} S^{\frac{N}{2}}-L m^{-\frac{N}{\beta}+\frac{N-2}{2 \beta}(q+1)} .
$$

\section{Prova do Lema 5.15.}

Seja $v=w+s \Phi_{\epsilon_{m}, m} \in W_{m, k} \oplus[0, \infty) \Phi_{\epsilon_{m}, m}$. Já sabemos que $I_{m}(v)=I_{m}(w)+I_{m}\left(s \Phi_{\epsilon_{m}, m}\right)$.

Na demonstração do Lema 5.12 vimos que, para $m$ suficientemente grande,

$$
I_{m}(w) \leq 0 \quad \forall w \in W_{m, k}
$$

Para estimarmos o termo $\Phi_{\epsilon_{m}, m}$, procedemos de modo análogo ao feito na demonstração do Lema 5.12 de modo a obtermos

$$
\begin{aligned}
I_{m}\left(s \Phi_{\epsilon_{m}, m}\right) \leq & \frac{s^{2}}{2} \int_{\Omega}\left|\nabla \Phi_{\epsilon_{m}, m}\right|^{2} d x-\frac{s^{2^{*}}}{2^{*}} \int_{\Omega} \Phi_{\epsilon_{m}, m}^{2^{*}} d x \\
& -\frac{\lambda}{2} \int_{\Omega}\left(s \Phi_{\epsilon_{m}, m}\right)^{2} d x-s \int_{\Omega} f \Phi_{\epsilon_{m}, m} d x .
\end{aligned}
$$

Assim, de (5.42), (5.43), usando os itens (a) e (b) do Lema 5.5 e lembrando que $\operatorname{supp} \Phi_{\epsilon_{m}, m} \subset B_{\frac{1}{m}}$, obtemos 


$$
\begin{aligned}
I_{m}(v) \leq & \frac{s^{2}}{2}\left(S^{\frac{N}{2}}+D_{1}\left(\epsilon_{m} m\right)^{N-2}\right)+\frac{s^{2^{*}}}{2^{*}}\left(-S^{\frac{N}{2}}+D_{2}\left(\epsilon_{m} m\right)^{N}\right) \\
& -\frac{\lambda}{2} \int_{\Omega}\left(s \Phi_{\epsilon_{m}, m}\right)^{2} d x+s \int_{B_{\frac{1}{m}}}\left|f \Phi_{\epsilon_{m}, m}\right| d x .
\end{aligned}
$$

Considerando $\epsilon_{m} m<\frac{1}{2}$, podemos usar o item (c) do Lema 5.5 para obtermos

$$
\begin{aligned}
I_{m}(v) \leq & \frac{s^{2}}{2}\left(S^{\frac{N}{2}}+D_{1}\left(\epsilon_{m} m\right)^{N-2}\right)+\frac{s^{2^{*}}}{2^{*}}\left(-S^{\frac{N}{2}}+D_{2}\left(\epsilon_{m} m\right)^{N}\right) \\
& -\frac{\lambda s^{2}}{2} D_{3} \epsilon_{m}^{2}+s \int_{B_{\frac{1}{m}}}\left|f \Phi_{\epsilon_{m}, m}\right| d x .
\end{aligned}
$$

Escolhendo $\mu>0$ de modo que $\frac{1}{\mu}+\frac{1}{r}+\frac{1}{2}=1$, podemos usar a desigualdade de Hölder generalizada no último termo da desigualdade acima, o que nos dá

$$
\begin{aligned}
\int_{B_{\frac{1}{m}}}\left|f \Phi_{\epsilon_{m}, m}\right| d x & \leq\|f\|_{r}\left\|\Phi_{\epsilon_{m}, m}\right\|_{2}\left(\int_{B_{\frac{1}{m}}} 1 d x\right)^{\frac{1}{\mu}} \\
& =\|f\|_{r}\left\|\Phi_{\epsilon_{m}, m}\right\|_{2}\left(\frac{\omega_{N}}{N}\right)^{\frac{1}{\mu}} m^{-\frac{N}{\mu}}
\end{aligned}
$$

Consideraremos a partir daqui $N \geq 5$. De (5.45) e do item (d) do Lema 5.5, obtemos

$$
\begin{aligned}
I_{m}(v) \leq & \frac{s^{2}}{2}\left(S^{\frac{N}{2}}+D_{1}\left(\epsilon_{m} m\right)^{N-2}\right)+\frac{s^{2^{*}}}{2^{*}}\left(-S^{\frac{N}{2}}+D_{2}\left(\epsilon_{m} m\right)^{N}\right) \\
& -\frac{\lambda s^{2}}{2} D_{3} \epsilon_{m}^{2}+s\|f\|_{r} D_{4} \epsilon_{m}\left(\frac{\omega_{N}}{N}\right)^{\frac{1}{\mu}} m^{-\frac{N}{\mu}} .
\end{aligned}
$$

Faça $c_{1}=\max \left\{D_{1}, D_{2}\right\}, c_{2}=\frac{\lambda}{2} D_{3}$ e $c_{3}=\|f\|_{r} D_{4}\left(\frac{\omega_{N}}{N}\right)^{\frac{1}{\mu}}$ e escreva

$$
\begin{aligned}
I_{m}(v) \leq & \frac{s^{2}}{2}\left(S^{\frac{N}{2}}+c_{1}\left(\epsilon_{m} m\right)^{N-2}\right)+\frac{s^{2^{*}}}{2^{*}}\left(-S^{\frac{N}{2}}+c_{1}\left(\epsilon_{m} m\right)^{N}\right) \\
& -c_{2} s^{2} \epsilon_{m}^{2}+c_{3} s \epsilon_{m} m^{-\frac{N}{\mu}} .
\end{aligned}
$$

Fixe $\Lambda>0$ tal que $\Lambda<\frac{S^{\frac{N}{2}}}{c_{1}}$ e considere $m$ suficientemente grande de modo que $\epsilon_{m} m<$ 
$\Lambda^{\frac{1}{N}}$, assim temos que

$$
I_{m}(v) \leq \frac{s^{2}}{2}\left(S^{\frac{N}{2}}+c_{1}\right)+\frac{s^{2^{*}}}{2^{*}}\left(-S^{\frac{N}{2}}+c_{1} \Lambda\right)+c_{3} s
$$

ou seja, mostramos até aqui que se $m$ for suficientemente grande, a restrição de $I_{m}$ a $W_{m, k} \oplus$ $[0, \infty) \Phi_{\epsilon_{m}, m}$ está abaixo da função $p:[0, \infty) \rightarrow \mathbb{R}$ dada por

$$
p(s)=-\frac{1}{2^{*}}\left(S^{\frac{N}{2}}-c_{1} \Lambda\right) s^{2^{*}}+\frac{1}{2}\left(S^{\frac{N}{2}}+c_{1}\right) s^{2}+c_{3} s
$$

Como $p(s) \underset{s \rightarrow \infty}{\longrightarrow}-\infty$, existe $M>1$, independente de $m$, tal que

$$
v=w+s \Phi_{\epsilon_{m}, m} \text { e } s \geq M \Rightarrow I_{m}(v) \leq p(s) \leq 0 .
$$

Estimaremos, a partir de agora, $v=w+s \Phi_{\epsilon_{m}, m} \in W_{m, k} \oplus[0, M] \Phi_{\epsilon_{m}, m}$. Voltando para a desigualdade (5.47), temos que

$$
\begin{aligned}
I_{m}(v) \leq & \left(\frac{s^{2}}{2}-\frac{s^{2^{*}}}{2^{*}}\right) S^{\frac{N}{2}}+c_{1}\left(\frac{s^{2}}{2}+\frac{s^{2^{*}}}{2^{*}}\left(\epsilon_{m} m\right)^{2}\right)\left(\epsilon_{m} m\right)^{N-2} \\
& -c_{2} s^{2} \epsilon_{m}^{2}+c_{3} s \epsilon_{m} m^{-\frac{N}{\mu}} .
\end{aligned}
$$

Considere $m$ suficientemente grande de modo que $\epsilon_{m} m<\Lambda^{\frac{1}{2}}$; substituindo $\epsilon_{m}$ por $m^{-\frac{1}{\beta}}$, obtemos

$$
\begin{aligned}
I_{m}(v) \leq & \left(\frac{s^{2}}{2}-\frac{s^{2^{*}}}{2^{*}}\right) S^{\frac{N}{2}}+c_{1}\left(\frac{s^{2}}{2}+\Lambda \frac{s^{2^{*}}}{2^{*}}\right) m^{\left(1-\frac{1}{\beta}\right)(N-2)} \\
& -c_{2} s^{2} m^{-\frac{2}{\beta}}+c_{3} s m^{-\left(\frac{1}{\beta}+\frac{N}{\mu}\right)}
\end{aligned}
$$

para todo $v \in W_{m, k} \oplus[0, M] \Phi_{\epsilon_{m}, m}$.

Observe que todos os expoentes de $m$ na desigualdade (5.49) são negativos.

Sejam $p_{m}, p_{0}:[0, M] \rightarrow \mathbb{R}$ funções dadas por

$$
p_{m}(s)=\left(\frac{s^{2}}{2}-\frac{s^{2^{*}}}{2^{*}}\right) S^{\frac{N}{2}}+c_{1}\left(\frac{s^{2}}{2}+\Lambda \frac{s^{2^{*}}}{2^{*}}\right) m^{\left(1-\frac{1}{\beta}\right)(N-2)}-c_{2} s^{2} m^{-\frac{2}{\beta}}+c_{3} s m^{-\left(\frac{1}{\beta}+\frac{N}{\mu}\right)}
$$




$$
p_{0}(s)=\left(\frac{s^{2}}{2}-\frac{s^{2^{*}}}{2^{*}}\right) S^{\frac{N}{2}}
$$

É fácil observar que $\max _{s \in[0, M]} p_{0}(s)=\frac{1}{N} S^{\frac{N}{2}}$ e que esse máximo é atingido quando $s=1$. Observe agora que

$$
\left|p_{m}(s)-p_{0}(s)\right| \leq c_{1}\left(\frac{s^{2}}{2}+\Lambda \frac{s^{2^{*}}}{2^{*}}\right) m^{\left(1-\frac{1}{\beta}\right)(N-2)}+c_{2} s^{2} m^{-\frac{2}{\beta}}+c_{3} s m^{-\left(\frac{1}{\beta}+\frac{N}{\mu}\right)}
$$

Como $s \in[0, M]$, temos que $p_{m} \underset{m \rightarrow \infty}{\longrightarrow} p_{0}$ uniformemente em $[0, M]$, implicando que

$$
\max _{s \in[0, M]} p_{m}(s) \underset{m \rightarrow \infty}{\longrightarrow} \max _{s \in[0, M]} p_{0}(s)=\frac{1}{N} S^{\frac{1}{N}}
$$

Como $p_{m}$ é contínua e $[0, M]$ é compacto, existe $s_{m} \in[0, M]$ tal que $p_{m}\left(s_{m}\right)=\max _{s \in[0, M]} p_{m}(s)$.

Observe que

$$
p_{m}(s) \leq\left(\frac{s^{2}}{2}-\frac{s^{2^{*}}}{2^{*}}\right) S^{\frac{N}{2}}+c_{1}\left(\frac{s^{2}}{2}+\Lambda \frac{s^{2^{*}}}{2^{*}}\right)+c_{3} s=p(s)
$$

onde $p:[0, M] \rightarrow \mathbb{R}$ é a função dado por (5.48). Agora note que $\max _{s \in[0, M]} p(s) \geq p(1)>\frac{1}{N} S^{\frac{N}{2}}$. Como $p(0)=0$, podemos considerar $\bar{M}=\min \left\{s \in(0, M): p(s) \geq \frac{1}{2 N} S^{\frac{1}{N}}\right\}$.

Para $m$ suficientemente grande segue de (5.50) que

$$
p_{m}\left(s_{m}\right) \geq \frac{1}{2 N} S^{\frac{N}{2}}
$$

por consequência, $s_{m} \geq \bar{M}$, do contrário, se algum $s_{m}<\bar{M}$, pela definição de $\bar{M}$, devemos ter $p\left(s_{m}\right)<\frac{1}{2 N} S^{\frac{N}{2}} ;$ como $p_{m}\left(s_{m}\right) \leq p\left(s_{m}\right)$, contrariamos $(5.51)$.

De acordo com o que foi visto logo acima, a desigualdade (5.49) se torna

$$
\begin{aligned}
I_{m}(v) & \leq\left(\frac{s_{m}^{2}}{2}-\frac{s_{m}^{2^{*}}}{2^{*}}\right) S^{\frac{N}{2}}+c_{1}\left(\frac{s_{m}^{2}}{2}+\Lambda \frac{s_{m}^{2^{*}}}{2^{*}}\right) m^{\left(1-\frac{1}{\beta}\right)(N-2)}-c_{2} s_{m}^{2} m^{-\frac{2}{\beta}}+c_{3} s_{m} m^{-\left(\frac{1}{\beta}+\frac{N}{\mu}\right)} \\
& \leq\left(\frac{s_{m}^{2}}{2}-\frac{s_{m}^{2^{*}}}{2^{*}}\right) S^{\frac{N}{2}}+c_{1}\left(\frac{M^{2}}{2}+\Lambda \frac{M^{2^{*}}}{2^{*}}\right) m^{\left(1-\frac{1}{\beta}\right)(N-2)}-c_{2} \bar{M}^{2} m^{-\frac{2}{\beta}}+c_{3} M m^{-\left(\frac{1}{\beta}+\frac{N}{\mu}\right)}
\end{aligned}
$$


Como $\frac{s_{m}^{2}}{2}-\frac{s_{m}^{2^{*}}}{2^{*}} \leq \frac{1}{N}$, para novas constantes positivas $c_{1}, c_{2}$ e $c_{3}$, obtemos

$$
I_{m}(v) \leq \frac{1}{N} S^{\frac{N}{2}}+c_{1} m^{\left(1-\frac{1}{\beta}\right)(N-2)}-c_{2} m^{-\frac{2}{\beta}}+c_{3} m^{-\left(\frac{1}{\beta}+\frac{N}{\mu}\right)} .
$$

Estaremos perto de concluir esta prova se conseguirmos mostrar que é possível escolher $\beta \in(0,1)$ de modo que $-\frac{2}{\beta}$ seja o maior expoente em $(5.52)$, ou seja, devemos mostrar que existe $\beta \in(0,1)$ tal que

$$
\left(1-\frac{1}{\beta}\right)(N-2)<-\frac{2}{\beta} \quad \text { e } \quad-\left(\frac{1}{\beta}+\frac{N}{\mu}\right)<-\frac{2}{\beta}
$$

Resolvendo (5.53) com respeito a $\beta$, obtemos

$$
\frac{\mu}{N}<\beta<\frac{N-4}{N-2}
$$

Devemos verificar agora em quais condições é possível escolher $\beta \in(0,1)$ de tal modo. É fácil verificar que $\frac{N-4}{N-2}<1$ qualquer que seja $N$. Observe agora que $\frac{1}{\mu}=\frac{1}{2}-\frac{1}{r}$ e lembre que $r>N$; logo, temos que

$$
\frac{1}{2}>\frac{1}{\mu}>\frac{1}{2}-\frac{1}{N}=\frac{1}{2^{*}}
$$

Isso nos sugere verificar quando vale $\frac{2^{*}}{N} \leq \frac{N-4}{N-2}$, pois, uma vez verificada tal desigualdade, como $\frac{\mu}{N}<\frac{2^{*}}{N}$, poderemos concluir que $\frac{\mu}{N}<\frac{N-4}{N-2}$. A simples conta a seguir nos mostra que devemos ter $N \geq 6$ :

$$
\frac{2}{N-2} \leq \frac{N-4}{N-2} \Leftrightarrow 2 \leq N-4 \Leftrightarrow N \geq 6
$$

É interessante observar que $\frac{\mu}{N}>\frac{2}{N}>\frac{N-4}{N-2}$ quando $N=5$, logo não seria possível termos $\frac{\mu}{N}<\frac{N-4}{N-2}$ para $N=5$ mesmo que escolhêssemos $r>N$ suficientemente grande.

Podemos agora dizer que se $N \geq 6$ e se $\beta \in\left(\frac{\mu}{N}, \frac{N-4}{N-2}\right)$, então as desigualdades em (5.53) estarão satisfeitas. Assim, podemos tomar $m$ suficientemente grande de modo que

$$
c_{1} m^{\left(1-\frac{1}{\beta}\right)(N-2)}+c_{3} m^{-\left(\frac{1}{\beta}+\frac{N}{\mu}\right)} \leq \frac{c_{2}}{2} m^{-\frac{2}{\beta}} .
$$


Da desigualdade acima e de (5.52), obtemos

$$
I_{m}(v) \leq \frac{1}{N} S^{\frac{N}{2}}-\frac{c_{2}}{2} m^{-\frac{2}{\beta}}
$$

para todo $v \in W_{m, k} \oplus[0, M] \Phi_{\epsilon_{m}, m}$. Se ainda $m$ for suficientemente grande de modo que $\frac{1}{N} S^{\frac{N}{2}}-\frac{c_{2}}{2} m^{-\frac{2}{\beta}}>0$, então (5.54) fica válida para todo $v \in W_{m, k} \oplus[0, \infty) \Phi_{\epsilon_{m}, m}$, já que se $s \geq M$, então $I_{m}(v) \leq 0$ como vimos mais acima.

Faça $L=\frac{c_{2}}{2}$. De $(5.33)$, segue que $c_{m} \leq \sup _{v \in Q_{R, m}^{\epsilon_{m}}} I_{m}(v)$. Como $Q_{R, m}^{\epsilon_{m}} \subset W_{m, k} \oplus[0, \infty) \Phi_{\epsilon_{m},}$, segue de (5.54) que

$$
c_{m} \leq \frac{1}{N} S^{\frac{N}{2}}-L m^{-\frac{2}{\beta}}
$$

Prova do Lema 5.16.

Seja $v=w+s \Phi_{\epsilon_{m}, m} \in W_{m, k} \oplus[0, \infty) \Phi_{\epsilon_{m}, m}$. Já sabemos que $I_{m}(v)=I_{m}(w)+I_{m}\left(s \Phi_{\epsilon_{m}, m}\right)$. Procedendo de modo idêntico ao feito no início da demonstração do Lema 5.15, no lugar de (5.43) obtemos

$$
\begin{aligned}
I_{m}\left(s \Phi_{\epsilon_{m}, m}\right) \leq & \frac{s^{2}}{2} \int_{\Omega}\left|\nabla \Phi_{\epsilon_{m}, m}\right|^{2} d x-\frac{s^{2^{*}}}{2^{*}} \int_{\Omega} \Phi_{\epsilon_{m}, m}^{2^{*}} d x \\
& -\int_{\Omega} G\left(x, s \Phi_{\epsilon_{m}, m}\right) d x-s \int_{\Omega} f \Phi_{\epsilon_{m}, m} d x
\end{aligned}
$$

onde descartamos o termo $-\frac{\lambda}{2} \int_{\Omega}\left(s \Phi_{\epsilon_{m}, m}\right)^{2} d x$, mas mantivemos o termo em $G$, pois precisaremos dele.

De $\left(g_{4}\right)$ segue que

$$
G(x, s) \geq \frac{D_{g}}{q+1} s^{q+1},
$$

com isso temos que

$$
-\int_{\Omega} G\left(x, s \Phi_{\epsilon_{m}, m}\right) d x \leq-\frac{D_{g}}{q+1} \int_{\Omega}\left|s \Phi_{\epsilon_{m}, m}\right|^{q+1} d x .
$$


Assim, de (5.56) e de (5.57), usando os itens (a), (b) do Lema 5.5 e considerando $m$ suficientemente grande para que usemos o item (c) do Lema 5.5, obtemos no lugar de (5.44)

$$
\begin{aligned}
I_{m}(v) \leq & \frac{s^{2}}{2}\left(S^{\frac{N}{2}}+D_{1}\left(\epsilon_{m} m\right)^{N-2}\right)+\frac{s^{2^{*}}}{2^{*}}\left(-S^{\frac{N}{2}}+D_{2}\left(\epsilon_{m} m\right)^{N}\right) \\
& -\frac{D_{g} s^{q+1}}{q+1} D_{3} \epsilon_{m}^{N-\frac{N-2}{2}(q+1)}+s \int_{B_{\frac{1}{m}}}\left|f \Phi_{\epsilon_{m}, m}\right| d x .
\end{aligned}
$$

Para estimarmos o último termo de (5.58), o procedimento é o mesmo que o feito no Lema 5.15 até obtermos (5.45). Usando agora o item (d) do Lema 5.5 e substituindo $\epsilon_{m}$ por $m^{-\frac{1}{\beta}}$, temos que

$$
\int_{B_{\frac{1}{m}}}\left|f \Phi_{\epsilon_{m}, m}\right| d x \leq \begin{cases}\|f\|_{r}\left(\frac{\omega_{5}}{5}\right)^{\frac{1}{\mu}} D(5) m^{-\left(\frac{1}{\beta}+\frac{5}{\mu}\right)}, & \text { se } \mathrm{N}=5 ; \\ \|f\|_{r}\left(\frac{\omega_{4}}{4}\right)^{\frac{1}{\mu}} D(4)\left|\log \left(m^{1-\frac{1}{\beta}}\right)\right|^{\frac{1}{2}} m^{-\left(\frac{1}{\beta}+\frac{4}{\mu}\right),} & \text { se } \mathrm{N}=4 ; \\ \|f\|_{r}\left(\frac{\omega_{3}}{3}\right)^{\frac{1}{\mu}} D(3) m^{-\left(\frac{1}{2 \beta}+\frac{3}{\mu}+\frac{1}{2}\right)}, & \text { se } \mathrm{N}=3 .\end{cases}
$$

Observe que

$$
\left|\log \left(m^{1-\frac{1}{\beta}}\right)\right|^{\frac{1}{2}}=\left|1-\frac{1}{\beta}\right|^{\frac{1}{2}}(\log m)^{\frac{1}{2}}
$$

recordando que $\frac{\log m}{m^{\eta}} \underset{m \rightarrow \infty}{\longrightarrow} 0$ para qualquer $\eta>0$, temos que para cada $\eta$ fixo podemos considerar $m \in \mathbb{N}$ suficientemente grande de modo que

$$
\left|\log \left(m^{1-\frac{1}{\beta}}\right)\right|^{\frac{1}{2}} \leq\left|1-\frac{1}{\beta}\right|^{\frac{1}{2}} m^{\frac{\eta}{2}}
$$

assim, para o caso $N=4$ temos que

$$
\int_{B_{\frac{1}{m}}}\left|f \Phi_{\epsilon_{m}, m}\right| d x \leq\|f\|_{r}\left(\frac{\omega_{4}}{4}\right)^{\frac{1}{\mu}} D(4)\left|1-\frac{1}{\beta}\right|^{\frac{1}{2}} m^{-\left(\frac{1}{\beta}+\frac{4}{\mu}-\frac{\eta}{2}\right)} .
$$

O valor de $\eta$ será escolhido mais a frente, suficientemente pequeno. 
Para abreviar a notação escreveremos

$$
\Theta_{N}(m)= \begin{cases}m^{-\left(\frac{1}{\beta}+\frac{5}{\mu}\right)}, & \text { se } \mathrm{N}=5 \\ m^{-\left(\frac{1}{\beta}+\frac{4}{\mu}-\frac{\eta}{2}\right)}, & \text { se } \mathrm{N}=4 \\ m^{-\left(\frac{1}{2 \beta}+\frac{3}{\mu}+\frac{1}{2}\right)}, & \text { se } \mathrm{N}=3\end{cases}
$$

Observe que podemos impor $\eta<8 / 2^{*}<8 / \mu$, assim todos os expoentes são negativos e logo teremos $\Theta_{N}(m) \rightarrow 0$ quando $m \rightarrow \infty$.

Faça $c_{3}=\|f\|_{r} \max \left\{\left(\frac{\omega_{5}}{5}\right)^{\frac{1}{\mu}} D(5), \quad\left(\frac{\omega_{4}}{4}\right)^{\frac{1}{\mu}} D(4)\left|1-\frac{1}{\beta}\right|^{\frac{1}{2}}, \quad\left(\frac{\omega_{3}}{3}\right)^{\frac{1}{\mu}} D(3)\right\} ;$ desse modo, de (5.59), (5.60) e (5.61), temos que

$$
\int_{B_{\frac{1}{m}}}\left|f \Phi_{\epsilon_{m}, m}\right| d x \leq c_{3} \Theta_{N}(m) .
$$

Fazendo agora $c_{1}=\max \left\{D_{1}, D_{2}\right\}, c_{2}=\frac{D_{g}}{q+1} D_{3}$ e combinando (5.58) e (5.62), no lugar de (5.47) obtemos

$$
\begin{aligned}
I_{m}(v) \leq & \frac{s^{2}}{2}\left(S^{\frac{N}{2}}+c_{1} m^{\left(1-\frac{1}{\beta}\right)(N-2)}\right)+\frac{s^{2^{*}}}{2^{*}}\left(-S^{\frac{N}{2}}+c_{1} m^{N\left(1-\frac{1}{\beta}\right)}\right) \\
& -c_{2} s^{q+1} m^{-\frac{N}{\beta}+\frac{N-2}{2 \beta}(q+1)}+c_{3} s \Theta_{N}(m) .
\end{aligned}
$$

Como $q+1<2^{*}$, todos os expoentes de $m$ na desigualdade (5.63) são negativos.

De forma análoga ao que foi feito na demonstração do Lema 5.15, podemos mostrar que existe $M>0$ tal que

$$
v=w+s \Phi_{\epsilon_{m}, m} \text { e } s \geq M \Rightarrow I_{m}(v) \leq 0
$$

e que se $v=w+s \Phi_{\epsilon_{m}, m} \operatorname{com} s \in[0, M]$, então, para novas constantes positivas $c_{1}, c_{2}$ e $c_{3}$, a desigualdade (5.63) se torna

$$
I_{m}(v) \leq \frac{1}{N} S^{\frac{N}{2}}+c_{1} m^{\left(1-\frac{1}{\beta}\right)(N-2)}-c_{2} m^{-\frac{N}{\beta}+\frac{N-2}{2 \beta}(q+1)}+c_{3} \Theta_{N}(m),
$$

ao invés daquela obtida em (5.52).

Estaremos perto de concluir esta prova se conseguirmos mostrar que é possível escolher 
$\beta \in(0,1)$ de modo que $-\frac{N}{\beta}+\frac{N-2}{2 \beta}(q+1)$ seja o maior expoente em (5.64), ou seja, devemos mostrar que existe $\beta \in(0,1)$ tal que

(A)

$$
\frac{N}{\beta}-\frac{N-2}{2 \beta}(q+1)<\left(\frac{1}{\beta}-1\right)(N-2)
$$

(B)

$$
\frac{N}{\beta}-\frac{N-2}{2 \beta}(q+1)< \begin{cases}\frac{1}{\beta}+\frac{5}{\mu}, & \text { se } N=5 ; \\ \frac{1}{\beta}+\frac{4}{\mu}-\frac{\eta}{2}, & \text { se } N=4 ; \\ \frac{1}{2 \beta}+\frac{3}{\mu}+\frac{1}{2}, & \text { se } N=3 .\end{cases}
$$

Antes de prosseguirmos, devemos observar que o fato de $\eta$ ainda não ter sido escolhido nos sugere verificar se é possível obtermos a desigualdade (B) no caso $N=4$ utilizando $\frac{1}{\beta}+\frac{4}{\mu}$ no lugar de $\frac{1}{\beta}+\frac{4}{\mu}-\frac{\eta}{2}$, caso seja possível, o resultado desejado será obtido com $\eta$ suficientemente pequeno.

\section{Verificação das desigualdades}

- Primeiro verificaremos em que condições podemos ter

$$
\frac{N}{\beta}-\frac{N-2}{2 \beta}(q+1)<\left(\frac{1}{\beta}-1\right)(N-2) \quad \text { e } \quad \frac{N}{\beta}-\frac{N-2}{2 \beta}(q+1)<\frac{1}{\beta}+\frac{N}{\mu} .
$$

Resolvendo a primeira desigualdade de (5.65) com respeito a $\beta$, obtemos

$$
\beta<-\frac{2}{N-2}+\frac{1}{2}(q+1)
$$

Como precisamos $\beta>0$, é necessário que a expressão acima seja positiva, o que equivale a

$$
q+1>\frac{4}{N-2}
$$

Antes de analisarmos as condições para a validade da segunda desigualdade de (5.65), devemos lembrar que $\mu<2^{*}$, $\log$ o temos que $\frac{1}{\beta}+\frac{N}{2^{*}}<\frac{1}{\beta}+\frac{N}{\mu}$; isso nos sugere verificar 
se

$$
\frac{N}{\beta}-\frac{N-2}{2 \beta}(q+1)<\frac{1}{\beta}+\frac{N}{2^{*}}
$$

Resolvendo a desigualdade acima com respeito a $\beta$, obtemos

$$
2 \frac{N-1}{N-2}-(q+1)<\beta
$$

Como precisamos $\beta<1$, é necessário que a expressão acima seja também menor que um, o que equivale a

$$
q+1>\frac{N}{N-2}
$$

A escolha de $\beta$ satisfazendo (5.66) e (5.69) só será possível se

$$
2 \frac{N-1}{N-2}-(q+1)<-\frac{2}{N-2}+\frac{1}{2}(q+1)
$$

que equivale a

$$
q+1>\frac{2}{3} 2^{*}
$$

Comparando (5.67), (5.70) e (5.71) vemos que, para $N=4,5$, a condição mais restritiva é (5.71).

Podemos então dizer que as desigualdades (A) e (B) para os casos $N=4$ e $N=5$ são válidas desde que escolhamos $q+1 \in\left(\frac{2}{3} 2^{*}, 2^{*}\right)$ e $\eta$ suficientemente pequeno.

- Queremos verificar agora em que condições podemos ter, no caso $N=3$,

$$
\begin{aligned}
& \frac{N}{\beta}-\frac{N-2}{2 \beta}(q+1)<\left(\frac{1}{\beta}-1\right)(N-2) \\
& \mathrm{e} \\
& \frac{N}{\beta}-\frac{N-2}{2 \beta}(q+1)<\frac{1}{2 \beta}+\frac{N}{\mu}+\frac{1}{2} .
\end{aligned}
$$

Como no item anterior, resolvendo a primeira desigualdade de (5.72) com respeito a 
$\beta$ obtemos (5.66) e a condição (5.67), que agora torna-se

$$
q+1>4
$$

Antes de analisarmos as condições para a validade da segunda desigualdade de (5.72), devemos lembrar que $\mu<2^{*}$, logo temos que $\frac{1}{2 \beta}+\frac{N}{2^{*}}+\frac{1}{2}<\frac{1}{2 \beta}+\frac{N}{\mu}+\frac{1}{2}$; isso nos sugere verificar se

$$
\frac{N}{\beta}-\frac{N-2}{2 \beta}(q+1)<\frac{1}{2 \beta}+\frac{N}{2^{*}}+\frac{1}{2},
$$

substituindo $N=3$ e resolvendo obtemos

$$
\beta>\frac{5-(q+1)}{2},
$$

o que nos dá a condição $q+1>3$, mais fraca de (5.73), para podermos respeitar $\beta<1$.

A escolha de $\beta$ será então possível se

$$
\frac{5-(q+1)}{2}<-\frac{2}{N-2}+\frac{1}{2}(q+1)=-2+\frac{1}{2}(q+1)
$$

o que nos leva a

$$
q+1>\frac{9}{2}
$$

mais estrita de (5.73).

Podemos então dizer que as desigualdades (A) e (B) são válidas para o caso $N=3$ desde que escolhamos $q+1 \in\left(\frac{9}{2}, 2^{*}\right)=\left(\frac{9}{2}, 6\right)$.

Concluindo, para $N=3,4,5$, é possível escolher $q$ e $\beta$ como no enunciado; feito isso, podemos tomar $m$ suficientemente grande de modo que

$$
c_{1} m^{\left(1-\frac{1}{\beta}\right)(N-2)}+c_{3} \Theta_{N}(m) \leq \frac{c_{2}}{2} m^{-\frac{N}{\beta}+\frac{N-2}{2 \beta}(q+1)} .
$$


Da desigualdade acima e de (5.64), obtemos

$$
I_{m}(v) \leq \frac{1}{N} S^{\frac{N}{2}}-\frac{c_{2}}{2} m^{-\frac{N}{\beta}+\frac{N-2}{2 \beta}(q+1)}
$$

para todo $v \in W_{m, k} \oplus[0, M] \Phi_{\epsilon_{m}, m}$. Se ainda $m$ for suficientemente grande de modo que $\frac{1}{N} S^{\frac{N}{2}}-\frac{c_{2}}{2} m^{-\frac{N}{\beta}+\frac{N-2}{2 \beta}(q+1)}>0$, então $(5.75)$ fica válida para todo $v \in W_{m, k} \oplus[0, \infty) \Phi_{\epsilon_{m}, m}$, já que se $s \geq M$, então $I_{m}(v) \leq 0$.

Faça $L=\frac{c_{2}}{2}$. De $(5.33)$, segue que $c_{m} \leq \sup _{v \in Q_{R, m}^{\epsilon_{m}}} I_{m}(v)$. Como $Q_{R, m}^{\epsilon_{m}} \subset W_{m, k} \oplus[0, \infty) \Phi_{\epsilon_{m},}$, segue de (5.75) que

$$
c_{m} \leq \frac{1}{N} S^{\frac{N}{2}}-L m^{-\frac{N}{\beta}+\frac{N-2}{2 \beta}(q+1)} .
$$

\subsection{Prova dos Teoremas 5.1 e 5.2}

Estamos agora perto de provar os Teoremas 5.1 e 5.2. Como mencionamos anteriormente, a prova desses teoremas se dará quando mostrarmos que a solução fraca $v_{0}$ é distinta da função $u_{t}-u_{t}^{m}$. Para verificar isso, compararemos o nível crítico $I_{m}\left(v_{0}\right)$ com o nível associado à função $u_{t}-u_{t}^{m}$ para certos $m \in \mathbb{N}$. Devemos, então, observar que

$$
\begin{aligned}
\left|I_{m}\left(u_{t}-u_{t}^{m}\right)\right| & =\left.\left|\frac{1}{2} \int_{\Omega}\right| \nabla\left(u_{t}-u_{t}^{m}\right)\right|^{2} d x-\frac{\lambda}{2} \int_{\Omega}\left(u_{t}-u_{t}^{m}\right)^{2} d x \mid \\
& \leq \frac{1}{2}\left\|u_{t}-u_{t}^{m}\right\|_{E}^{2}+\frac{\lambda}{2}\left\|u_{t}-u_{t}^{m}\right\|_{2}^{2} \\
& \leq C\left\|u_{t}-u_{t}^{m}\right\|_{E}^{2} ;
\end{aligned}
$$

pelo Lema 5.3 obtemos

$$
\left|I_{m}\left(u_{t}-u_{t}^{m}\right)\right| \leq C m^{-N}
$$

A seguir, exibiremos algumas estimativas que serão muito úteis para fazer a comparação entre o nível crítico $I_{m}\left(v_{0}\right)$ e o nível associado a $u_{t}-u_{t}^{m}$.

- Pelo item (i) do TEOREMA DE MERGULHO DE SOBOLEV, temos que $v_{n}+u_{t}^{m}, v_{0}+$ 
$u_{t}^{m} \in L^{2^{*}}(\Omega)$. Como $\left\{v_{n}\right\}_{n \in \mathbb{N}}$ é limitada em $E$ temos que $\left\|v_{n}+u_{t}^{m}\right\|_{2^{*}}$ também é limitada. Como $v_{n} \underset{n \rightarrow \infty}{\longrightarrow} v_{0}$ q.t.p., pelo Lema de Brezis-Lieb (veja em particular o Corolário C.4) temos que

$$
\int_{\Omega}\left(v_{0}+u_{t}^{m}\right)_{+}^{2^{*}} d x=\lim _{n \rightarrow \infty}\left(\int_{\Omega}\left(v_{n}+u_{t}^{m}\right)_{+}^{2^{*}} d x-\int_{\Omega}\left(v_{n}-v_{0}\right)_{+}^{2^{*}} d x\right) .
$$

- Da condição $\left(g_{3}\right)$ temos

$$
G(x, s) \leq \frac{C_{g}}{p+1}|s|^{p+1}
$$

Pelo Lema B.3, é contínuo o operador de Nemytskii $N_{G}: L^{p+1}(\Omega) \rightarrow L^{1}(\Omega)$ dado por

$$
N_{G}(v)=G(\cdot, v) .
$$

Como $v_{n} \underset{n \rightarrow \infty}{\longrightarrow} v_{0}$ em $L^{p+1}(\Omega) \mathrm{e}$

$$
\begin{array}{r}
\left|\int_{\Omega}\left[G\left(x,\left(v_{n}+u_{t}^{m}\right)_{+}\right)-G\left(x,\left(v_{0}+u_{t}^{m}\right)_{+}\right)\right] d x\right| \leq \\
\leq \int_{\Omega}\left|G\left(x,\left(v_{n}+u_{t}^{m}\right)_{+}\right)-G\left(x,\left(v_{0}+u_{t}^{m}\right)_{+}\right)\right| d x \\
\quad=\left\|N_{G}\left(\left(v_{n}+u_{t}^{m}\right)_{+}\right)-N_{G}\left(\left(v_{0}+u_{t}^{m}\right)_{+}\right)\right\|_{1},
\end{array}
$$

obtemos

$$
\int_{\Omega} G\left(x,\left(v_{0}+u_{t}^{m}\right)_{+}\right) d x=\lim _{n \rightarrow \infty} \int_{\Omega} G\left(x,\left(v_{n}+u_{t}^{m}\right)_{+}\right) d x .
$$

- Seja $h: \Omega \times \mathbb{R} \rightarrow \mathbb{R}$ uma função dada por

$$
h(x, s)=g\left(x, s_{+}\right) s .
$$


Da condição $\left(g_{3}\right)$ temos

$$
|h(x, s)| \leq C_{g}|s|^{p+1}
$$

Pelo Lema B.3 é contínuo o operador de Nemytskii $N_{h}: L^{p+1}(\Omega) \rightarrow L^{1}(\Omega)$ dado por

$$
N_{h}(v)=h(\cdot, v)=g\left(\cdot, v_{+}\right) v
$$

Como $v_{n} \underset{n \rightarrow \infty}{\longrightarrow} v_{0}$ em $L^{p+1}(\Omega)$, segue que $\left\|N_{h}\left(v_{n}+u_{t}^{m}\right)-N_{h}\left(v_{0}+u_{t}^{m}\right)\right\|_{1} \underset{n \rightarrow \infty}{\longrightarrow} 0$, ou seja,

$$
\int_{\Omega}\left|g\left(x,\left(v_{n}+u_{t}^{m}\right)_{+}\right)\left(v_{n}+u_{t}^{m}\right)-g\left(x,\left(v_{0}+u_{t}^{m}\right)_{+}\right)\left(v_{0}+u_{t}^{m}\right)\right| d x \underset{n \rightarrow \infty}{\longrightarrow} 0 .
$$

o que nos dá

$$
\int_{\Omega} g\left(x,\left(v_{0}+u_{t}^{m}\right)_{+}\right) v_{0}=\lim _{n \rightarrow \infty} \int_{\Omega} g\left(x,\left(v_{n}+u_{t}^{m}\right)_{+}\right) v_{n} d x
$$

- Como $u_{t}^{m} \in C(\bar{\Omega})$, temos

$$
\begin{gathered}
\left|\int_{\Omega}\left(v_{n}+u_{t}^{m}\right)_{+}^{2^{*}-1} u_{t}^{m} d x-\int_{\Omega}\left(v_{0}+u_{t}^{m}\right)_{+}^{2^{*}-1} u_{t}^{m} d x\right| \leq \\
\leq\left\|u_{t}^{m}\right\|_{\infty} \int_{\Omega}\left|\left(v_{n}+u_{t}^{m}\right)_{+}^{2^{*}-1}-\left(v_{0}-u_{t}^{m}\right)_{+}^{2^{*}-1}\right| d x \\
\quad=\left\|u_{t}^{m}\right\|_{\infty}\left\|\left(v_{n}+u_{t}^{m}\right)_{+}^{2^{*}-1}-\left(v_{0}-u_{t}^{m}\right)_{+}^{2^{*}-1}\right\|_{1} .
\end{gathered}
$$

Como $v_{n} \underset{n \rightarrow \infty}{\longrightarrow} v_{0}$ em $L^{2^{*}-1}$, obtemos

$$
\int_{\Omega}\left(v_{0}+u_{t}^{m}\right)_{+}^{2^{*}-1} u_{t}^{m} d x=\lim _{n \rightarrow \infty} \int_{\Omega}\left(v_{n}+u_{t}^{m}\right)_{+}^{2^{*}-1} u_{t}^{m} d x
$$


- Como

$$
\left.\left|\int_{\Omega}\right| \nabla v_{n}\right|^{2} d x-\int_{\Omega}\left|\nabla v_{0}\right|^{2} d x-\int_{\Omega}\left|\nabla\left(v_{n}-v_{0}\right)\right|^{2} d x|=2| \int_{\Omega} \nabla v_{n} \cdot \nabla v_{0} d x-\int_{\Omega}\left|\nabla v_{0}\right|^{2} d x \mid
$$

e $v_{n} \rightarrow_{n \rightarrow \infty} v_{0}$ em $E$, segue que

$$
\int_{\Omega}\left|\nabla v_{0}\right|^{2} d x=\lim _{n \rightarrow \infty}\left(\int_{\Omega}\left|\nabla v_{n}\right|^{2} d x-\int_{\Omega}\left|\nabla\left(v_{n}-v_{0}\right)\right|^{2} d x\right)
$$

Iremos agora utilizar as estimativas obtidas acima.

Observe que

$$
\begin{aligned}
I_{m}\left(v_{0}\right)-I_{m}\left(v_{n}\right)=- & \frac{1}{2}\left(\int_{\Omega}\left|\nabla v_{n}\right|^{2} d x-\int_{\Omega}\left|\nabla v_{0}\right|^{2} d x\right)+\frac{\lambda}{2}\left(\int_{\Omega} v_{n}^{2} d x-\int_{\Omega} v_{0}^{2} d x\right) \\
& +\frac{1}{2^{*}}\left(\int_{\Omega}\left(v_{n}+u_{t}^{m}\right)_{+}^{2^{*}} d x-\int_{\Omega}\left(v_{0}+u_{t}^{m}\right)_{+}^{2^{*}} d x\right) \\
& +\int_{\Omega} G\left(x,\left(v_{n}+u_{t}^{m}\right)_{+}\right) d x-\int_{\Omega} G\left(x,\left(v_{0}+u_{t}^{m}\right)_{+}\right) d x \\
& +\left(F-F_{m}\right)\left(v_{n}-v_{0}\right) ;
\end{aligned}
$$

logo, de (5.78), (5.79), (5.82), do fato de $v_{n} \underset{n \rightarrow \infty}{\longrightarrow} v_{0}$ em $L^{2}(\Omega)$, e lembrando que $I_{m}\left(v_{n}\right) \underset{n \rightarrow \infty}{\longrightarrow}$ $c_{m}$, obtemos

$$
I_{m}\left(v_{0}\right)-c_{m}=\lim _{n \rightarrow \infty}\left(-\frac{1}{2} \int_{\Omega}\left|\nabla\left(v_{n}-v_{0}\right)\right|^{2} d x+\frac{1}{2^{*}} \int_{\Omega}\left(v_{n}-v_{0}\right)_{+}^{2^{*}} d x\right)
$$

Lembrando que $I_{m}^{\prime}\left(v_{0}\right) \cdot v_{0}=0$, não é difícil verificar que

$$
\begin{aligned}
I_{m}^{\prime}\left(v_{n}\right) \cdot v_{n}= & I_{m}^{\prime}\left(v_{n}\right) \cdot v_{n}-I^{\prime}\left(v_{0}\right) \cdot v_{0} \\
= & \int_{\Omega}\left|\nabla v_{n}\right|^{2} d x-\int_{\Omega}\left|\nabla v_{0}\right|^{2} d x-\lambda\left(\int_{\Omega} v_{n}^{2} d x-\int_{\Omega} v_{0}^{2} d x\right) \\
& -\int_{\Omega}\left(v_{n}+u_{t}^{m}\right)_{+}^{2^{*}} d x+\int_{\Omega}\left(v_{0}+u_{t}^{m}\right)_{+}^{2^{*}} d x \\
& +\int_{\Omega}\left(v_{n}+u_{t}^{m}\right)_{+}^{2^{*}-1} u_{t}^{m} d x-\int_{\Omega}\left(v_{0}+u_{t}^{m}\right)_{+}^{2^{*}-1} u_{t}^{m} d x \\
& -\int_{\Omega} g\left(x,\left(v_{n}+u_{t}^{m}\right)_{+}\right) v_{n} d x+\int_{\Omega} g\left(x,\left(v_{0}+u_{t}^{m}\right)_{+}\right) v_{0} \\
& -\left(F-F_{m}\right)\left(v_{n}-v_{0}\right) ;
\end{aligned}
$$


logo, de (5.78), (5.80), (5.81), (5.82), do fato de $v_{n} \underset{n \rightarrow \infty}{\longrightarrow} v_{0}$ em $L^{2}(\Omega)$ e lembrando que $\left|I_{m}^{\prime}\left(v_{n}\right) \cdot v_{n}\right| \underset{n \rightarrow \infty}{\longrightarrow} 0$, obtemos

$$
\lim _{n \rightarrow \infty}\left(\int_{\Omega}\left|\nabla\left(v_{n}-v_{0}\right)\right|^{2} d x-\int_{\Omega}\left(v_{n}-v_{0}\right)_{+}^{2^{*}} d x\right)=0
$$

Os limites em (5.83) e (5.84) serão fundamentais para a prova do Proposição a seguir:

\section{Proposição 5.17.}

É possivel escolher $\beta$ de maneira que a solução fraca $v_{0}$ de $\left(P_{m}\right)$ seja distinta de $u_{t}-u_{t}^{m}$.

\section{Prova.}

Pelo Lema 5.13 a sequência $a_{n}=\left\|v_{n}-v_{0}\right\|_{E}^{2}$ é limitada, logo existe uma subsequência, que continuaremos denotando por $a_{n}$, tal que $a_{n} \underset{n \rightarrow \infty}{\longrightarrow} K \geq 0$.

Se $K=0$, então $v_{n} \underset{n \rightarrow \infty}{\longrightarrow} v_{0}$ em $E$ e de (5.34) segue que $I_{m}\left(v_{0}\right)=c_{m}$. Escolhendo $m \in \mathbb{N}$ suficientemente grande na estimativa (5.77) de modo que $\mathrm{Cm}^{-N}<\alpha$, onde $\alpha>0$ é o número obtido no Lema 5.11, obtemos

$$
I_{m}\left(u_{t}-u_{t}^{m}\right) \leq C m^{-N}<\alpha \leq c_{m}=I_{m}\left(v_{0}\right)
$$

do que segue $v_{0} \not \equiv u_{t}-u_{t}^{m}$.

Suporemos então que $K>0$. Usando a definição da melhor constante para o mergulho de Sobolev

$$
\begin{aligned}
\left\|v_{n}-v_{0}\right\|_{E}^{2} & \geq S\left(\int_{\Omega}\left|v_{n}-v_{0}\right|^{2^{*}} d x\right)^{\frac{2}{2^{*}}} \\
& \geq S\left(\int_{\Omega}\left(v_{n}-v_{0}\right)_{+}^{2^{*}} d x\right)^{\frac{2}{2^{*}}}
\end{aligned}
$$

fazendo, agora, $n \rightarrow \infty$ e usando (5.84) obtemos $K \geq S K^{\frac{2}{2^{*}}}=S K^{\frac{N-2}{N}}$, ou seja, $K \geq S^{\frac{N}{2}}$.

Fazendo $n \rightarrow \infty$ em (5.83), temos

$$
\begin{aligned}
I_{m}\left(v_{0}\right)-c_{m} & =-\frac{1}{2} K+\frac{1}{2^{*}} K \\
& =-\frac{1}{N} K .
\end{aligned}
$$


Utilizando os Lemas 5.15 e 5.16 em (5.86), obtemos

$$
I_{m}\left(v_{0}\right)=c_{m}-\frac{1}{N} K \leq \begin{cases}\frac{S^{\frac{N}{2}}-K}{N}-L m^{-\frac{2}{\beta}}, & \text { se } N \geq 6 \\ \frac{S^{\frac{N}{2}}-K}{N}-L m^{-\frac{N}{\beta}+\frac{N-2}{2 \beta}(q+1)}, & \text { se } N=3,4,5\end{cases}
$$

Por outro lado, utilizando (5.77),

$$
I_{m}\left(u_{t}-u_{t}^{m}\right) \geq-C m^{-N}
$$

Para que $v_{0}$ seja distinto de $u_{t}-u_{t}^{m}$ precisaremos então que

$$
-C m^{-N}> \begin{cases}\frac{S^{\frac{N}{2}}-K}{N}-L m^{-\frac{2}{\beta}}, & \text { se } N \geq 6 ; \\ \frac{S^{\frac{N}{2}}-K}{N}-L m^{-\frac{N}{\beta}+\frac{N-2}{2 \beta}(q+1)}, & \text { se } N=3,4,5 .\end{cases}
$$

Como $K \geq S^{\frac{N}{2}}$, isso será verdade para $m$ grande desde que

$$
N> \begin{cases}\frac{2}{\beta}, & \text { se } N \geq 6 \\ \frac{N}{\beta}-\frac{N-2}{2 \beta}(q+1), & \text { se } N=3,4,5 .\end{cases}
$$

Se $N \geq 6$, como $\beta>\frac{\mu}{N}>\frac{2}{N}$ (veja o Lema 5.15), a condição está satisfeita.

Se $N=3,4,5$, resolvendo em $\beta$, a condição se torna

$$
\beta>1-\frac{N-2}{2 N}(q+1)
$$

Comparando com o Lema 5.16, vemos que $\beta$ pode ser escolhido assim desde que

$$
1-\frac{N-2}{2 N}(q+1)<\frac{1}{2}(q+1)-\frac{2}{N-2},
$$

que é equivalente a

$$
q+1>\frac{N^{2}}{(N-2)(N-1)}
$$


Agora observe que para $N=4,5$

$$
\frac{N^{2}}{(N-2)(N-1)} \leq \frac{2}{3} \frac{2 N}{N-2}=\frac{2}{3} 2^{*}
$$

e para $N=3$

$$
\frac{N^{2}}{(N-2)(N-1)}=\frac{9}{2}
$$

logo, para $N=3,4,5$, a condição (5.90) está satisfeita nas hipóteses do Teorema 5.2.

Concluímos então que $v_{0} \not \equiv u_{t}-u_{t}^{m}$.

Devido à Proposição 5.17, podemos agora dizer que para escolhas apropriadas de $\beta \in$ $(0,1)$ o problema $\left(P_{m}\right)$ tem uma solução fraca $v_{0} \not \equiv u_{t}-u_{t}^{m}$; equivalentemente, o problema $P$ admite uma segunda solução fraca na forma $v_{0}+u_{t}^{m}$, distinta da solução negativa $u_{t}$. Assim ficam provados os Teoremas 5.1 e 5.2. 
Apêndice $\mathcal{A}$

\section{Resultados Importantes}

Neste seção enunciaremos sem provar alguns resultados que foram necessários para a realização deste trabalho.

A seguinte proposição nos dá uma caracterização variacional para os autovalores do laplaciano. A prova para tais estimativas pode ser encontrada, por exemplo, na demonstração do Teorema 12.26 de [Bie10].

\section{Proposição A.1.}

Seja $k \in \mathbb{N}$. Considere $E=W_{k} \oplus X_{k}$ onde $W_{k}=\left\langle\phi_{i}\right\rangle_{i=1}^{k}$ e $X_{k}=W_{k}^{\perp} ;$ temos então que

(a) $\|u\|_{E}^{2} \leq \lambda_{k}\|u\|_{2}^{2}, \forall u \in W_{k}$;

(b) $\lambda_{k+1}\|u\|_{2}^{2} \leq\|u\|_{E}^{2}, \forall u \in X_{k}$

Os teoremas de imersão de Sobolev são de grande importância. A demonstração de tais teoremas pode ser encontrada em [Bie10] ou em [Ada75].

\section{Teorema A.2 (TEOREMA DE MERGULHO DE SOBOLEV).}

Sejam $k, N \in \mathbb{N}$ e $p \geq 1$. Seja $\Omega \subset \mathbb{R}^{N}$ um dominio limitado com fronteira suave; então

(i) se $k p<N$, então $W^{k, p}(\Omega) \hookrightarrow L^{q}(\Omega)$ para todo $q \in\left[1, p^{*}\right]$, onde $p^{*}=\frac{p N}{N-p}$. A imersão é compacta se $q \in\left[1, p^{*}\right)$;

(ii) se $k p=N$, então $W^{k, p}(\Omega) \hookrightarrow L^{q}(\Omega)$ para todo $q \in[1, \infty)$; 
(iii) se $N<p k$, então $W^{k, p}(\Omega) \hookrightarrow C^{m, \beta}(\bar{\Omega})$ para todo $0 \leq m<k-\frac{N}{p}$, onde

$$
\begin{array}{lr}
0<\beta \leq\left[\frac{N}{p}\right]+1-\frac{N}{p} & \text { se } \frac{N}{p} \text { nã for inteiro, } \\
0<\beta<1 & \text { se } \frac{N}{p} \text { for inteiro. }
\end{array}
$$

\section{Observação A.3.}

Seja $\Omega \subset \mathbb{R}^{N}$ um dominio qualquer. Do exemplo 5.25 em [Adar5] podemos concluir que se $k p<N$, então não é possível o mergulho $W_{0}^{k, p}(\Omega) \hookrightarrow L^{q}(\Omega)$ para todo $q \in\left(p^{*}, \infty\right]$. Já do exemplo 5.26 da mesma referência podemos concluir que se $k p=N$, então não é possível o mergulho $W^{k, p}(\Omega) \hookrightarrow L^{\infty}(\Omega)$. Em particular, se $k=1$ e $p=2$, então não temos $E \subset L^{\infty}(\Omega)$ para todo $N \geq 2$.

Os dois seguintes teoremas podem ser encontrados com suas respectivas demonstrações em [GT01].

\section{Teorema A.4 (TEOREMA DA ALTERNATIVA DE FREDHOLM).}

Sejam $X$ um espaço vetorial normado e $T: X \rightarrow X$ um operador linear compacto. Então vale uma das alternativas

- $a$ equação

$$
x-T x=0
$$

admite uma solução não trivial $x \in X$;

- dado $y \in X$ existe um único $x \in X$ satisfazendo

$$
x-T x=y
$$

e a aplicação $(I d-T)^{-1}$, cuja existência neste caso está assegurada, é linear e limitada.

\section{Teorema A.5 (TEOREMA DE LAX - MILGRAN).}

Seja $H$ um espaço de Hilbert. Se a aplicação $B: H \times H \rightarrow H$ for uma forma bilinear limitada e coerciva, então dado $F \in H^{\prime}$ (dual topológico de $H$ ) existe um único $f \in H$ tal que

$$
B(x, f)=F(x) \quad \forall x \in H .
$$


Enunciaremos agora um resultado de regularidade de soluções fracas de problemas elípticos; tal resultado é, na verdade, um caso bem particular do Teorema 8.2 provado em [Agm59].

\section{Teorema A.6 (TEOREMA DE REGULARIDADE).}

Sejam $\Omega$ um aberto limitado de $\mathbb{R}^{N}$ de classe $C^{\infty}, K>0$ e $1<r<\infty$. Então existe uma constante $C$, dependendo apenas de $N, \Omega, p$ e $K$ tal que, se $u_{0} \in L^{q}(\Omega)$, com $1<q<\infty$, é solução fraca de

$$
\begin{cases}-\Delta u+c u=f, & \text { em } \Omega \\ u=0, & \text { sobre } \partial \Omega\end{cases}
$$

com $|c| \leq K$ e $f \in L^{r}(\Omega)$, então $u_{0} \in W^{2, r}(\Omega) \cap W_{0}^{1, r}(\Omega)$ e

$$
\left\|u_{0}\right\|_{2, r} \leq C\left(\left\|u_{0}\right\|_{r}+\|f\|_{r}\right) .
$$




\section{Apêndice $\mathcal{B}$ \\ Operador de Nemytskii}

Nesta seção falaremos um pouco sobre o operador de Nemytskii e alguns teoremas que envolvem tal noção. Para maiores detalhes consulte [dF89].

No que segue, $\Omega$ denotará um subconjunto aberto e limitado de $\mathbb{R}^{N}, N \geq 1$.

\section{Definição B.1.}

Uma função $f: \Omega \times \mathbb{R} \rightarrow \mathbb{R}$ é dita uma função de Carathéodory se para cada $s \in \mathbb{R} a$ aplicação $x \mapsto f(x, s)$ é Lebesgue mensurável em $\Omega$ e se para quase todo $x \in \Omega$ a função $s \mapsto f(x, s)$ é contínua.

Seja $\mathcal{M}$ o conjunto de todas as funções mensuráveis $u: \Omega \rightarrow \mathbb{R}$.

\section{Teorema B.2.}

Se $f: \Omega \times \mathbb{R} \rightarrow \mathbb{R}$ é uma função de Carathyéodory, então está bem definida a aplicação $N_{f}: \mathcal{M} \rightarrow \mathcal{M}$ dada por

$$
N_{f}(u)=f(\cdot, u)
$$

ou seja, sempre que $u \in \mathcal{M}, N_{f}(u): \Omega \rightarrow \mathbb{R}$ será uma função mensurável dada por

$$
N_{f}(u)(x)=f(x, u(x))
$$

A aplicação $N_{f}$ definida no teorema acima é chamada de operador de Nemytskii. Para nós é interessante saber quando $N_{f}$ transforma continuamente um espaço $L^{p}(\Omega)$ em outro. 
Teorema B.3.

Seja $f: \Omega \times \mathbb{R} \rightarrow \mathbb{R}$ uma função de Carathéodory. Suponha que existam uma constante $c>0, b \in L^{q}(\Omega), 1 \leq q \leq \infty$, e $r>0$ tais que

$$
|f(x, s)| \leq c|s|^{r}+b(x) \quad \forall x \in \Omega, \quad \forall s \in \mathbb{R} .
$$

Então o operador de Nemytskii $N_{f}: L^{q r}(\Omega) \rightarrow L^{q}(\Omega)$ dado por (B.1) está bem definido e, além disso, essa aplicação é continua e limitada.

Segue imediatamente do Teorema B.3 que se $u_{n} \underset{n \rightarrow \infty}{\longrightarrow} u_{0}$ em $L^{q r}(\Omega)$, então $f\left(\cdot, u_{n}\right) \underset{n \rightarrow \infty}{\longrightarrow}$ $f\left(\cdot, u_{0}\right)$ em $L^{q}(\Omega)$. 


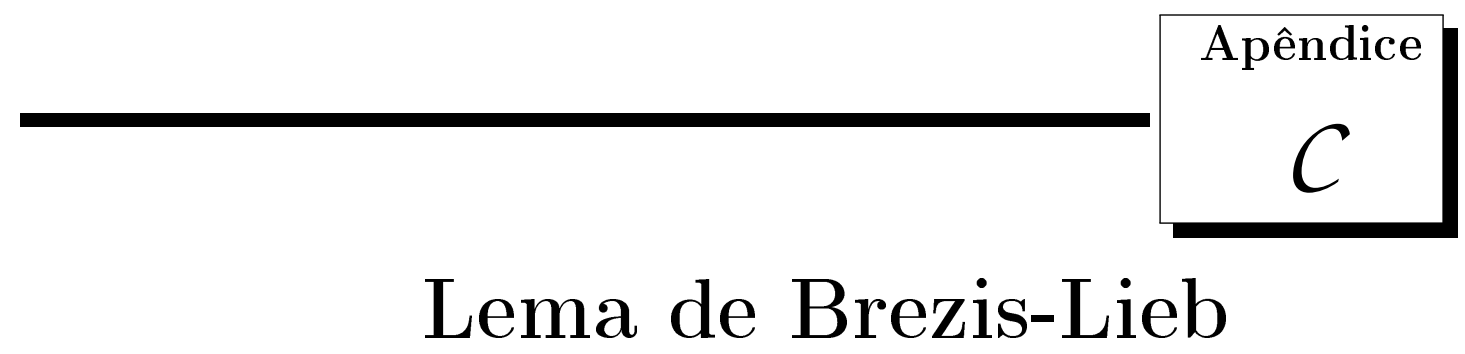

Nesta seção enunciaremos o Lema de Brezis-Lieb (vide [BL83]), na sua forma mais geral, e em seguida apresentaremos um caso particular. Para esse fim, enunciaremos antes uma definição e provaremos um lema que nos auxiliará na demonstração do caso particular.

\section{Definição C.1.}

Dizemos que uma função $F: \mathbb{R} \rightarrow \mathbb{R}$ é p-homogênea, $p>0$, se

$$
F(t x)=t^{p} F(x) \quad \forall t \geq 0, \quad \forall x \in \mathbb{R}
$$

Observe que se $F: \mathbb{R} \rightarrow \mathbb{R}$ é p-homogênea, então segue da definição que $F(0)=0$.

\section{Lema C.2.}

Seja $F: \mathbb{R} \rightarrow \mathbb{R}$ uma função contínua, não-negativa e p-homogênea. Então dado $\epsilon>0$, existe $C_{\epsilon}>0$ tal que

$$
|F(a+b)-F(a)| \leq \epsilon|a|^{p}+C_{\epsilon}|b|^{p} \quad \forall a, b \in \mathbb{R} .
$$

\section{Prova.}

Dado $\epsilon>0$, pela continuidade da $F$, existe $\widetilde{\delta}_{\epsilon}>0$ tal que

$$
|F(a+b)-F(a)| \leq \epsilon \quad \forall|b| \leq \widetilde{\delta}_{\epsilon}
$$

Considere $\delta_{\epsilon}=\min \left\{1, \widetilde{\delta}_{\epsilon}\right\}$ e defina $M=\max _{x \in[-2,2]} F(x)$ e $C_{\epsilon}=\frac{2 M}{\delta_{\epsilon}^{p}}$. 
O caso $a, b=0$ é trivial. Se supormos $a=0$ e $b \neq 0$, como $\left|\frac{b}{|b|}\right|=1$, temos

$$
\left|F\left(\frac{b}{|b|}\right)\right| \leq M \leq \frac{2 M}{\delta_{\epsilon}^{p}}
$$

logo, pela p-homogeneidade da $F$, temos

$$
|F(b)| \leq C_{\epsilon}|b|^{p}
$$

Suporemos agora $a, b \neq 0$.

Se $1 \leq \frac{|b|}{|a|}$, como $\left|\frac{a}{|b|}+\frac{b}{|b|}\right| \leq 2$, temos

$$
\left|F\left(\frac{a}{|b|}+\frac{b}{|b|}\right)-F\left(\frac{a}{|b|}\right)\right| \leq 2 M \leq \frac{2 M}{\delta_{\epsilon}^{p}} \leq \epsilon \frac{|a|^{p}}{|b|^{p}}+\frac{2 M}{\delta_{\epsilon}^{p}} .
$$

A p-homogeneidade da $F$ nos dá

$$
|F(a+b)-F(a)| \leq \epsilon|a|^{p}+C_{\epsilon}|b|^{p} .
$$

Se $\delta_{\epsilon} \leq \frac{|b|}{|a|} \leq 1$, como $\left|\frac{a}{|a|}+\frac{b}{|a|}\right| \leq 2$, temos

$$
\left|F\left(\frac{a}{|a|}+\frac{b}{|a|}\right)-F\left(\frac{a}{|a|}\right)\right| \leq 2 M=\frac{2 M}{\delta_{\epsilon}^{p}} \delta_{\epsilon}^{p} \leq \frac{2 M}{\delta_{\epsilon}^{p}} \frac{|b|^{p}}{|a|^{p}} \leq \epsilon+\frac{2 M}{\delta_{\epsilon}^{p}} \frac{|b|^{p}}{|a|^{p}} .
$$

A p-homogeneidade da $F$ nos dá

$$
|F(a+b)-F(a)| \leq \epsilon|a|^{p}+C_{\epsilon}|b|^{p} .
$$

Se $\frac{|b|}{|a|} \leq \delta_{\epsilon}$, então

$$
\left|F\left(\frac{a}{|a|}+\frac{b}{|a|}\right)-F\left(\frac{a}{|a|}\right)\right| \leq \epsilon \leq \epsilon+\frac{2 M}{\delta_{\epsilon}^{p}} \frac{|b|^{p}}{|a|^{p}}
$$

A p-homogeneidade da $F$ nos dá

$$
|F(a+b)-F(a)| \leq \epsilon|a|^{p}+C_{\epsilon}|b|^{p} .
$$


Assumiremos aqui o Lema de Brezis-Lieb cuja demonstração pode ser encontrada em [BL83].

\section{Lema C.3. (Brezis-Lieb)}

Seja $j: \mathbb{R} \rightarrow \mathbb{R}$ uma função contínua tal que $j(0)=0$. Suponha que para cada $\epsilon>0$ suficientemente pequeno existam funções continuas e não negativas $\varphi_{\epsilon}, \psi_{\epsilon}: \mathbb{R} \rightarrow \mathbb{R}$ tais que

$$
|j(a+b)-j(a)| \leq \epsilon \varphi_{\epsilon}(a)+\psi_{\epsilon}(b) \quad \forall a, b \in \mathbb{R} .
$$

Considere $f_{n}, f, g_{n}: \Omega \rightarrow \mathbb{R}$ funções mensuráveis satisfazendo $f_{n}=f+g_{n}$ e suponha que

(i) $g_{n} \underset{n \rightarrow \infty}{\longrightarrow} 0$ q.t.p. em $\Omega$;

(ii) $j(f) \in L^{1}(\Omega)$;

(iii) $\int_{\Omega} \varphi_{\epsilon}\left(g_{n}(x)\right) d x \leq C<\infty$ para alguma constante $C$ que não dependa de $\epsilon$ e $n$;

(iv) $\int_{\Omega} \psi_{\epsilon}(f(x)) d x<\infty \forall \epsilon>0$

Então,

$$
\int_{\Omega}\left|j\left(f+g_{n}\right)-j\left(g_{n}\right)-j(f)\right| d x \underset{n \rightarrow \infty}{\longrightarrow} 0
$$

Dos Lemas C.2 e C.3 segue um resultado bastante útil para este trabalho:

\section{Corolário C.4. (Caso particular do Lema de Brezis-Lieb)}

Seja $p \in[1, \infty)$. O Lema de Brezis-Lieb é válido para o caso especial em que $j: \mathbb{R} \rightarrow \mathbb{R}$ é dada por

$$
j(t)=t_{+}^{p}
$$

\section{Prova.}

É fácil observar que a função $j: \mathbb{R} \rightarrow \mathbb{R}$ dada por (C.3) é contínua, não negativa e phomogênea. Logo, pelo Lema C.2 temos que

$$
\left|(a+b)_{+}^{p}-a_{+}^{p}\right|=|j(a+b)-j(a)| \leq \epsilon|a|^{p}+C_{\epsilon}|b|^{p} \quad \forall a, b \in \mathbb{R} .
$$


Considerando $\varphi_{\epsilon}, \psi_{\epsilon}: \mathbb{R} \rightarrow \mathbb{R}$, dadas por

$$
\varphi_{\epsilon}(t)=|t|^{p} \quad \psi_{\epsilon}(t)=C_{\epsilon}|t|^{p}
$$

temos que $j$ satisfaz (C.2) e isso completa a prova.

Em outras palavras, o Lema C.4 nos diz que se $f_{n}, f \in L^{p}(\Omega)$ são tais que

- $f_{n} \underset{n \rightarrow \infty}{\longrightarrow} f$ q.t.p. em $\Omega$,

- $\left\|f_{n}\right\|_{L^{p}} \leq C$, para alguma constante $C$ que não dependa de $n$,

então

$$
\int_{\Omega}(f)_{+}^{p} d x=\lim _{n \rightarrow \infty}\left(\int_{\Omega}\left(f_{n}\right)_{+}^{p} d x-\int_{\Omega}\left(f_{n}-f\right)_{+}^{p} d x\right) .
$$




\section{Bibliografia}

[Ada75] R. A. Adams, Sobolev spaces, Academic Press [A subsidiary of Harcourt Brace Jovanovich, Publishers], New York-London, 1975, Pure and Applied Mathematics, Vol. 65 .

[Agm59] S. Agmon, The $L_{p}$ approach to the Dirichlet problem. I. Regularity theorems, Ann. Scuola Norm. Sup. Pisa (3) 13 (1959), 405-448.

[Bie10] R. J. Biezuner, Notas de Aula - Equações Diferenciais Parciais I/II, 2010.

[BL83] H. Brézis and E. Lieb, A relation between pointwise convergence of functions and convergence of functionals, Proc. Amer. Math. Soc. 88 (1983), no. 3, 486-490.

[BN83] H. Brézis and L. Nirenberg, Positive solutions of nonlinear elliptic equations involving critical Sobolev exponents, Comm. Pure Appl. Math. 36 (1983), no. 4, $437-477$.

[CR02] M. Calanchi and B. Ruf, Elliptic equations with one-sided critical growth, Electron. J. Differential Equations (2002), No. 89, 21 pp. (electronic).

[dF89] D. G. de Figueiredo, Lectures on the Ekeland variational principle with applications and detours, Tata Institute of Fundamental Research Lectures on Mathematics and Physics, vol. 81, Published for the Tata Institute of Fundamental Research, Bombay; by Springer-Verlag, Berlin, 1989.

[DFJ99] D. G. De Figueiredo and Y. Jianfu, Critical superlinear Ambrosetti-Prodi problems, Topol. Methods Nonlinear Anal. 14 (1999), no. 1, 59-80. 
[Gho93] N. Ghoussoub, Duality and perturbation methods in critical point theory, Cambridge Tracts in Mathematics, vol. 107, Cambridge University Press, Cambridge, 1993, With appendices by David Robinson.

[GL87] N. Garofalo and F.-H. Lin, Unique continuation for elliptic operators: a geometricvariational approach, Comm. Pure Appl. Math. 40 (1987), no. 3, 347-366.

[GR97] F. Gazzola and B. Ruf, Lower-order perturbations of critical growth nonlinearities in semilinear elliptic equations, Adv. Differential Equations 2 (1997), no. 4, 555572.

[GT01] D. Gilbarg and N. S. Trudinger, Elliptic partial differential equations of second order, Classics in Mathematics, Springer-Verlag, Berlin, 2001, Reprint of the 1998 edition.

[MW89] J. Mawhin and M. Willem, Critical point theory and Hamiltonian systems, Applied Mathematical Sciences, vol. 74, Springer-Verlag, New York, 1989.

[Poh65] S. I. Pohožaev, On the eigenfunctions of the equation $\Delta u+\lambda f(u)=0$, Dokl. Akad. Nauk SSSR 165 (1965), 36-39.

[RS86] B. Ruf and P. N. Srikanth, Multiplicity results for superlinear elliptic problems with partial interference with the spectrum, J. Math. Anal. Appl. 118 (1986), no. 1, 1523.

[Str08] M. Struwe, Variational methods, third ed., Ergebnisse der Mathematik und ihrer Grenzgebiete. 3. Folge. A Series of Modern Surveys in Mathematics [Results in Mathematics and Related Areas. 3rd Series. A Series of Modern Surveys in Mathematics], vol. 34, Springer-Verlag, Berlin, 2008, Applications to nonlinear partial differential equations and Hamiltonian systems. 